\title{
ON THE NATURE AND IMPLICATIONS OF STARBURST-DRIVEN GALACTIC SUPERWINDS
}

\author{
Timothy M. HeCKMAN, ${ }^{1,2,3}$ LeE ARMus, ${ }^{1,3}$ AND GeORGe K. Miley ${ }^{2,3,4}$ \\ Received 1989 July 18; accepted 1990 May 10
}

\begin{abstract}
We presented optical spectroscopic data on the ionized nebulae associated with 14 galaxies that are strong far-infrared emitters ("far-IR galaxies," or "FIRGs"). This sample includes the three nearest FIRGs (M82, NGC 253, and NGC 4945), three of the nearest and more famous examples of very luminous FIRGs with bolometric luminosities of nearly $10^{12} L_{\odot}$ (NGC 3690/IC 694, Arp 220, and NGC 6240), and the most luminous known FIRG (IRAS $00182-7112, L_{\mathrm{IR}} \approx 10^{13} L_{\odot}$ ). We find that our data provide both qualitative and quantitative support for the "superwind" model in which the kinetic energy provided by supernovae and winds from massive stars in a central starbust drives a large-scale outflow that can shock heat and accelerate ambient interstellar or circumgalactic gas.

For the three nearest FIRGs (all disk galaxies seen nearly edge-on) we find that clear kinematic signature of an outflow along the galaxy's minor axis. Kiloparsec-scale regions well out of the disk are characterized by double emission-line profiles with line splitting of $200-600 \mathrm{~km} \mathrm{~s}^{-1}$. The emission-line gas evidently lies along the surface of an expanding bubble or outflowing conelike structure. Similar structures are present in the edge-on FIRG NGC 3079 and in the brighter " $\mathrm{H} \alpha$ bubble" in Arp 220. The gas in both NGC 6240 and IRAS 00182-7112 is very violently disturbed kinematically (lines having FWHM $600-1000 \mathrm{~km} \mathrm{~s}^{-1}$ over galaxy-scale regions). The sizes, velocities, and kinematic/morphological structure of these outflows agree well with the superwind model, which predicts that the wind fluid will inflate an expanding cavity in the ISM of the FIRG, with optical line emission coming from the thin outer shell of shock-heated, swept-up ambient gas.

We also find that the FIRG nebulae are highly overpressured (by three to four orders of magnitude) relative to the ISM of the Milky Way, with the pressures dropping systematically with distance from the nucleus. The radius at which a fiducial pressure occurs is proportional to the square root of the IR luminosity. In the best-studied cases, the shapes of the radial density/pressure profiles are shallow at radii of less than few hundred parsecs and steep at radii greater than $1 \mathrm{kpc}$. These results are all in excellent agreement with theoretical predictions of the superwind model (relatively constant, static thermal pressure inside the region of energy/mass injection and ram pressure proportional to the star-formation rate at large radii). The shapes of the pressure profiles are inconsistent with a wind driven by an AGN. The sizes of the mass/energy injection regions derived from the pressure profiles agree well with directly measured starburst radii, and the measured pressures at large radii are close to the predicted ram pressures for starburst-driven superwinds.

We find that superwinds are energetically adequate to power both the observed optical and X-ray nebulae, and also show that the relative emission-line intensities and their radial variations are consistent with ionization by wind-driven shocks, but not with photoionization by normal O stars or an AGN.

We discuss the possible astrophysical implication of superwinds. At the present epoch, a typical superwind may inject $10^{8} M_{\odot}$ of metals and $10^{58}$ ergs into the intergalactic medium (IGM) over its estimated lifetime of $10^{7} \mathrm{yr}$. The local luminosity function for FIRGs implies that about $10^{9} M_{\odot}$ of metals and $10^{59} \mathrm{ergs}$ could be injected, on average, per $L_{*}$ galaxy over a Hubble time, even with no cosmic evolution in the superwind rate. Superwinds may play important or even dominant roles in the metal enrichment and heating of both the intracluster medium and general intergalactic medium. Superwinds may make an important contibution to the cosmic X-ray background, and their relationship to the QSO absorption-line phenomenon should be explored. We also speculate that superwinds may represent a phase in the evolution of a FIRG to a QSO/AGN and of a disk-disk galaxy merger to an elliptical galaxy. Finally, we emphasize that powerful FIRGs may be reasonable approximations to galaxies in formation. If so, then superwinds may be important during the process of galaxy formation, with particular relevance to the "explosions" or the "feedback" mechanisms suggested by Ostriker, Cowie, Ikeuchi, Dekel and Silk, White, and others.
\end{abstract}

Subject headings: galaxies: intergalactic medium — galaxies: internal motions — galaxies: interstellar matter — galaxies: structure - infrared: sources

\footnotetext{
${ }^{1}$ Department of Physics and Astronomy, Johns Hopkins University.

${ }^{2}$ Space Telescope Science Institute.

${ }^{3}$ Visiting observers at the Kitt Peak National Observatory and the Cerro Tololo Inter-American Observatory of the National Optical Astronomy Observatories, operated by AURA, Inc., for the National Science Foundation.

${ }^{4}$ Leiden Observatory, Leiden University.
} 


\section{INTRODUCTION}

\section{a) Theory of Superwinds}

Galactic-scale "superwinds" driven by the collective effect of supernovae and winds from massive stars have a rich legacy in theoretical astrophysics. They have been invoked as the source by which a metal-enriched intracluster medium was created and heated (e.g., De Young 1978). Some have suggested that superwinds played a critical role in the formation and early evolution of both dwarf galaxies (e.g., Dekel and Silk 1986) and massive elliptical galaxies (Mathews 1989). They have also been proposed as the mechanism that underlies both the mass/metallicity relation between galaxies and the metallicity/radius relation within galaxies: the deeper the local gravitational potential, the more difficult to drive a wind and hence the greater the ability of the locale to retain metal-rich processed material (e.g., Franx 1990). They may be important contributors to the cosmic X-ray background (Bookbinder et al. 1980) and figure directly in recent evolutionary scenarios that seek to unite the far-IR galaxies and traditional types of AGNs and QSOs (see Sanders et al. 1988). It has even been suggested that the winds/explosions associated with the formation of galaxies may have played some role in the formation of structure in the universe (Ostriker and Cowie 1981).

There is a rich astrophysical literature relevant to the physics of superwinds, since this basic physics is common to many problems such as supernova explosions, stellar winds, winds from AGNs, "explosions" in the early universe, etc. (an exhaustive review of the problem can be found in Ostriker and McKee 1988). Figure 1 is a "cartoon" illustrating the essential structural features and dynamical evolution of a superwind occurring in a plane-parallel atmosphere (galaxy disk) and is based on the numerical simulations by Tomisaka and Ikeuchi (1988, hereafter TI).

As discussed by Chevalier and Clegg (1985, hereafter CC), a superwind results when the kinetic energy in the ejecta supplied by supernovae and winds from massive stars in a nuclear starburst is efficiently thermalized. This means that the collisions of intersecting SNRs/winds convert the kinetic energy of the ejecta into thermal energy via shocks, with little energy lost to radiation because of the very high postshock temperatures and low densities. The collective effect of the supernovae and stellar winds then creates a "bubble" of very hot $\left(\approx 10^{8} \mathrm{~K}\right)$ gas with a pressure that is much larger than that of its surroundings. As the bubble expands and sweeps up ambient gas, it will rapidly enter the "snowplow" or radiative phase, which occurs when the radiative cooling time of the ambient material that it is shock heating becomes shorter than the wind's expansion time scale.

The snowplow phase has been modeled by Castor, McCray, and Weaver (1975, hereafter CMW), Weaver et al. (1977), and others in the context of wind-blown bubbles surrounding massive stars. Five radially stratified components are present at this time (see Fig. 1a): (1) an innermost region in which the

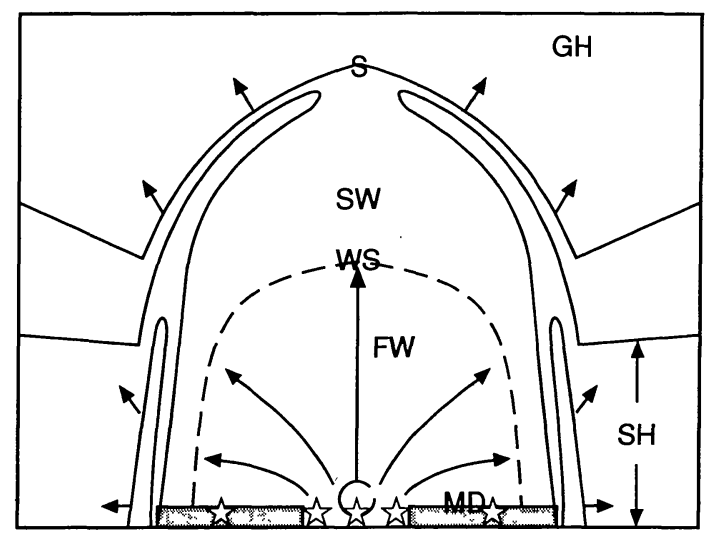

FIG. $1 a$

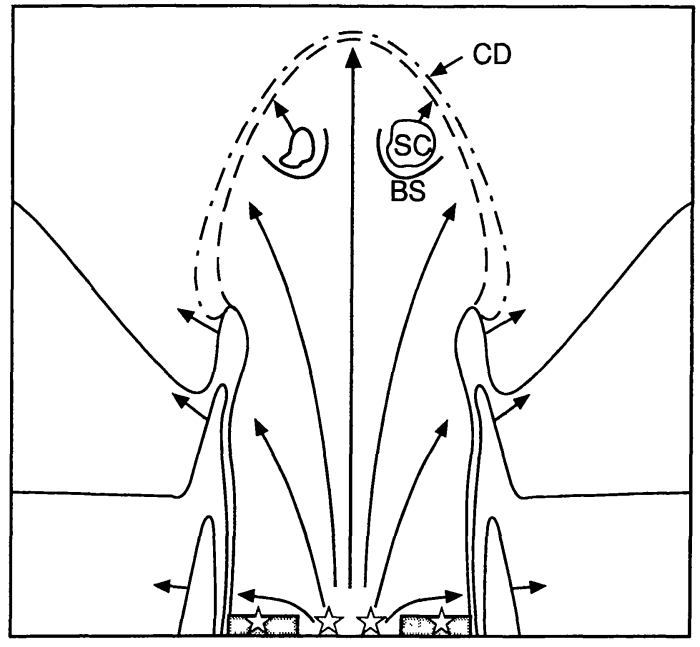

FIG. $1 b$

FIG. 1.-Schematic diagrams showing the essential features predicted for two evolutionary phases of a superwind expanding into the gaseous halo of a

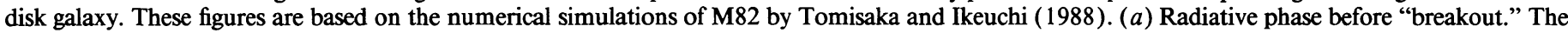

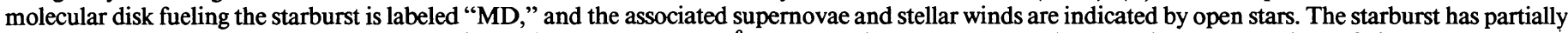

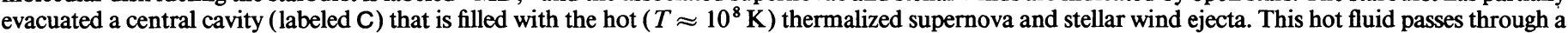

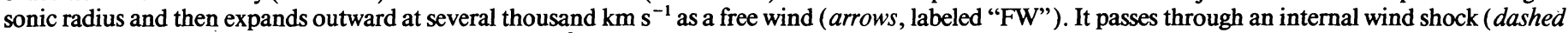

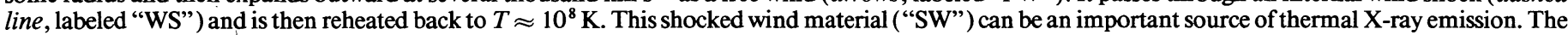

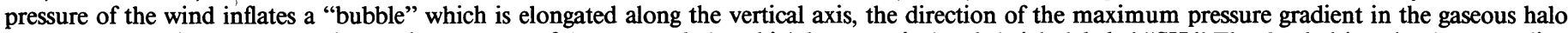

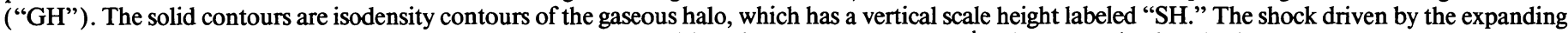

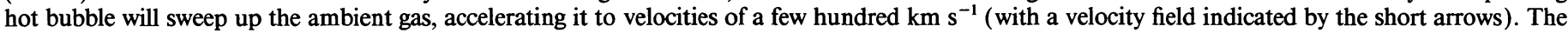

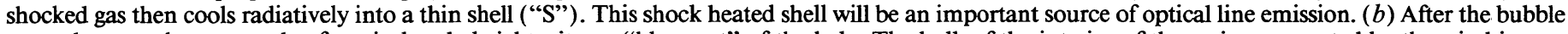

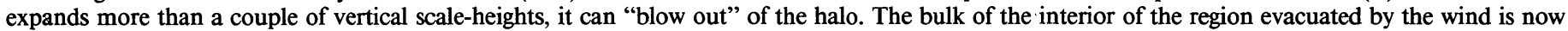

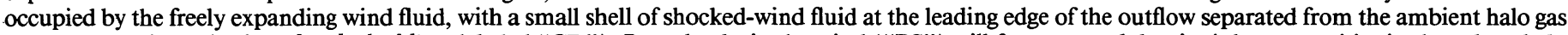

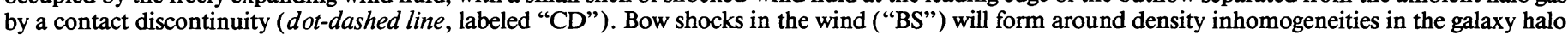

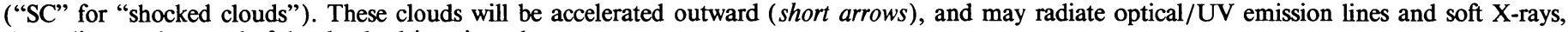
depending on the speed of the shocks driven into them. 
mass and energy are injected and thermalized (the starburst); (2) a region of the supersonic wind with $v=$ several thousand $\mathrm{km} \mathrm{s}^{-1} ;(3)$ a hot $\left(T \approx 10^{8} \mathrm{~K}\right) \mathrm{X}$-ray-emitting region of wind material that has been decelerated and heated as it passed through an internal shock; (4) a thin and derise shell of shocked, swept-up ambient gas being pushed outward at one to several hundred $\mathrm{km} \mathrm{s}^{-1}$ (a strong source of optical line emission); and (5) the undisturbed ambient gas. Since the dynamical evolution depends only on the rate at which energy or momentum is injected into the wind and on the density distribution of the ambient medium, kinematic data can be used to determine these momentum or energy injection rates.

It is likely that once the windblown bubble has a diameter that is a few times the scale height of the ambient medium, it will evolve from the "snowplow" phase into the "blowout" phase as the swept-up shell accelerates outward and fragments via Rayleigh-Taylor instabilities. The wind can then propagate out into the intergalactic medium (see Fig. 1b; TI; Mac Low, McCray, and Norman 1989, hereafter MMN; Norman and Ikeuchi 1989). Optical and X-ray emission from the wind in this phase will come from obstacles (clouds, shell fragments) that are immersed in and shock heated by the outflow, and additional X-ray emission may come from internal shocks in the wind fluid. Some considerable fraction of the entrained/ shock-heated ambient material may be accelerated to high enough velocities to escape the galaxy along with wind fluid (i.e., the wind may sweep out a substantial portion of the galaxy's ISM).

During the "blowout" phase (essentially free expansion), the pressure, temperature, density, and velocity of the wind are simple functions of radius ( see CC). The central region where energy is injected by the starburst is interior to the sonic point of the wind, and hence the pressure there is dominated by thermal pressure. This region is roughly isobaric with a pressure that is set by both its radius and the effective rate at which momentum is injected into it. The outer region is dominated by the ram pressure of the wind, and the wind is characterized by a constant velocity. This region therefore has (ram) pressures that drop as the radius squared. The ram pressure at a given radius depends only on the effective momentum injection rate for the superwind. Thus, the radial pressure profiles can be used to determine the rate of momentum injection in the wind and the characteristic dimension of the region of momentum injection (the starburst).

\section{b) Observational Signatures of Superwinds}

Until recently, galactic superwinds remained purely theoretical entities. However, optical and X-ray observations of the nearest starburst galaxies have provided suggestive evidence that superwinds do indeed exist (see Heckman, Armus, and Miley 1987, hereafter HAM; McCarthy, Heckman, and van Breugel 1987, hereafter MHV; Fabbiano, Heckman, and Keel 1990; Bland and Tully 1988; Fabbiano 1988; Meurer 1989).

Guided by the above summary of the theoretical structure and evolution of a superwind, the evidence for the existence of superwinds is as follows:

Morphological: Large-scale (several $\mathrm{kpc}$ to several tens of $\mathrm{kpc}$ ) optical emission-line and X-ray nebulae oriented perpendicular to the stellar disks have been discovered in several nearby starburst galaxies (e.g., Watson, Stanger, and Griffiths
1984; Fabbiano and Trinchieri 1984; MHV; HAM; Fabbiano, Heckman, and Keel 1990).

Physical: The nebulae in several starburst galaxies have optical emission-line ratios suggestive of material that has been shock heated (HAM; MHV; Fabbiano, Heckman, and Keel (1990); Armus, Heckman, and Miley 1989). The best-studied such nebula (in M82) has a radial pressure profile (MHV) and radial X-ray surface brightness profile (Fabbiano 1988), whose shape and amplitude is in good agreement with the theoretical predictions for a superwind in the blowout phase. Moreover, the mass and thermal energy of the M82 X-ray nebula are also consistent with the model (Fabbiano 1988; Schaaf $e t$ al. 1989).

Kinematic: The kinematics of the nebulae in M82 and the starbursting dwarf galaxy NGC 1705 implies that in both galaxies the optical emission-line gas lies on the surface of an expanding bubble or biconic structure with outflow velocities of a few hundred $\mathrm{km} \mathrm{s}^{-1}$ (Bland and Tully 1988; Meurer 1989). The kinematic signature of this outflow is a large (few $\mathrm{kpc}$ ) region with double-peaked emission-line profiles.

The overall weight of this evidence seems thoroughly convincing in the case of M82, but does not yet establish that superwinds are a characteristic feature of starburst galaxies.

\section{c) The Present Paper}

The new observations that we will report and discuss in this paper were therefore undertaken to determine the ubiquity of superwinds in starburst galaxies and to place the superwind model on a more secure observational foundation. To this end, we have searched for three types of evidence of superwinds in a sample of IR-luminous galaxies: clear kinematic signatures of outflows, the kind of systematic radial drop in gas pressure seen in the M82 nebula, and emission-line spectra that are consistent with shock heating.

In the present paper, we report on the kinematics of the ionized gas in seven prototypical far-IR galaxies ("FIRGs"): M82, NGC 253, NGC 4945, NGC 3079, NGC 6240, Arp 220, and IRAS 00182-7112. The first three galaxies are the nearest FIRGs to us, and each has an IR luminosity of about $10^{44} \mathrm{ergs}$ $\mathrm{s}^{-1}$. Fortuitously, all three are disk galaxies seen nearly edgeon. Both their proximity and special orientation allow us to study the "superwinds" in a detailed and relatively unambiguous fashion. NGC 3079 has a similar IR luminosity, an edge-on orientation, and is still relatively nearby. Arp 220 and NGC 6240 are the nearest and best-studied examples of very powerful FIRGs $\left(L_{\mathrm{IR}} \approx 10^{12} L_{\odot}\right)$, while IRAS $00182-7112$ is the most FIR-powerful galaxy yet discovered $\left(L_{\mathrm{IR}}\right.$ nearly $10^{13}$ $L_{\odot}$ ). We will also present radial electron density profiles (and estimated gas pressure profiles) for 12 FIRGs in Armus, Heckman, and Miley (1989) having bright, spatially extended emission in the [S II] $\lambda \lambda 6717,6731$ lines (M82, NGC 253, NGC 1222, NGC 1614, NGC 3079, NGC 3256, NGC 3690, NGC 4194, NGC 4945, NGC 6240, Mrk 266, and Mrk 273). We have also searched for clues to the ionization mechanism for the gas through the use of standard emission-line ratio diagnostics for all 14 FIRGs listed above (the salient properties of which are summarized in Table 1 ).

In $\S$ II we briefly describe the observations and data reduction. In $\S$ III we present the results on the kinematics, density/ pressure profiles, and emission-line ratios. Section IV is a dis- 
TABLE 1

Basic Properties of THe Observed Galaxies

\begin{tabular}{|c|c|c|c|c|c|}
\hline $\begin{array}{c}\text { Galaxy } \\
\text { (1) }\end{array}$ & $\begin{array}{c}D \\
(2)\end{array}$ & $\begin{array}{c}S \\
(3)\end{array}$ & $\underset{(4)}{\log L_{\mathrm{IR}}}$ & $\begin{array}{c}L_{\mathrm{IR}} / L_{B} \\
\quad(5)\end{array}$ & $\begin{array}{c}f_{60} / f_{100} \\
(6)\end{array}$ \\
\hline IRAS 00182-7112 ......... & 1300 & 4200 & $\approx 12.9$ & $\approx 200$ & 1.09 \\
\hline NGC 253 ........................ & 3.0 & 14.5 & 10.46 & 3.5 & 0.54 \\
\hline NGC $1222 \ldots \ldots \ldots \ldots \ldots \ldots$ & 36 & 174 & 10.46 & 6 & 0.86 \\
\hline NGC $1614 \ldots \ldots \ldots \ldots \ldots \ldots$ & 62 & 300 & 11.41 & 12 & 1.09 \\
\hline M82 .......................... & 3.0 & 14.5 & 10.52 & 10 & 0.94 \\
\hline NGC 3079 .................... & 16 & 79 & 10.49 & 2.2 & 0.51 \\
\hline NGC 3256 ................... & 34 & 163 & 11.48 & 11 & 0.78 \\
\hline NGC 3690 ..................... & 41 & 200 & 11.29 & 13 & 1.00 \\
\hline 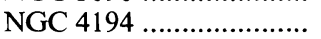 & 36 & 174 & 10.87 & 6 & 0.94 \\
\hline NGC 4945 ..................... & 4.6 & 22.3 & 10.61 & 9 & 0.42 \\
\hline 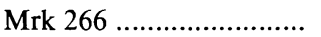 & 116 & 541 & 11.37 & 8 & 0.65 \\
\hline Mrk 273 ........................ & 154 & 746 & 12.10 & 29 & 1.16 \\
\hline Arp $220 \ldots \ldots \ldots \ldots \ldots \ldots \ldots \ldots$ & 73 & 350 & 12.12 & 150 & 0.96 \\
\hline NGC 6240 ..................... & 97 & 472 & 11.72 & 45 & 0.90 \\
\hline
\end{tabular}

NoTE.-Key to cols. (2)-(6) follows:

Col. (2).-Distance adopted (in Mpc). These distances correspond to $c z / H_{0}$, with $H_{0}=75 \mathrm{~km} \mathrm{~s}^{-1} \mathrm{Mpc}^{-1}$ except for the very nearby galaxies NGC 253, M82, and NGC 4945.

Col. (3). - Angular scale (pc arcsec ${ }^{-1}$ ) implied by the distances in col. (2). We adopt $q_{0}=0$.

Col. (4).- The log of the total infrared luminosity (in $L_{\odot}$ ). These are computed using the distances in col. (2) and the total infrared fluxes derived by Rice et al. 1988 or Soifer et al. 1987 from two-temperature and one-temperature dust emission models, respectively. Note that the luminosity given for NGC 3690 refers to that galaxy alone: the total for the entire system of NGC 3690 + IC 694 is 2.7 times larger (see Joy et al. 1989; Nakagawa et al. 1989). For IRAS 00182-7112, the quoted luminosity is estimated by using the dust-emission model fitted by Soifer et al. (1987) to NGC 1614, then scaled to the redshift and IR flux of IRAS 00182-7112 (the two galaxies have similar mid/far-IR spectral energy distributions).

Col. (5).- Ratio of the far-IR luminosity (col. [4]) and the blue luminosity. The latter are derived from blue magnitudes on the $B_{T}$ scale generally taken from the Revised Shapley-Ames Catalog (Sandage and Tammann 1981, hereafter RSA) or the Second Reference Catalogue of Bright Galaxies (de Vaucouleurs, de Vaucouleurs, and Corwin 1976, hereafter RC2). Data on IRAS 00182-7112 come from Armus, Heckman, and Miley (1987). Magnitudes were then $K$-corrected and corrected for foreground Galactic extinction using the $A_{B}$ values given in the RSA or RC2, but were not corrected for internal extinction.

Col. (6).-Ratio of the IRAS flux densities in the $60 \mu \mathrm{m}$ and $100 \mu \mathrm{m}$ bands, taken from Rice $e t$ al. 1988 , Soifer et al. 1987, and Lonsdale et al. 1985, in order of preference.

cussion of these results, $\S \mathrm{V}$ is an exploration of their implications, and $\S \mathrm{VI}$ is a summary of our principal conclusions.

\section{OBSERVATIONS AND DATA REDUCTION}

Our data were obtained with the $4 \mathrm{~m}$ telescopes at KPNO and CTIO. The RC spectrograph with the TI2 CCD was used at KPNO, and the Cassegrain spectrograph with a GEC CCD was used at CTIO. The relevant details are summarized in Table 2.

The CTIO data were bias-subtracted and then flat-fielded with the NOAO "CCDPROC" software by using exposures of a dome screen illuminated with a quartz lamp. Because of the color dependence of the CCD response, a series of dome flats were taken with a variety of different grating tilts. The appropriate dome flat was then used to flatten each galaxy spectrum. The KPNO data were bias-subtracted and flat-fielded in IRAF. Both a dome flat (to remove the low spatial frequency structure-e.g., vignetting along the slit) and exposures taken of a quartz lamp mounted in the RC spectrograph (to remove the high spatial frequency structure) were used. The latter were taken immediately before and after each galaxy spectrum.

In all cases $\mathrm{HeNeAr}$ spectra were taken before and after the galaxy spectra. These were later averaged and then used for wavelength calibration and geometrical rectification in the LONGSLIT software package in IRAF. The spectra were then sky-subtracted interactively in IRAF. The KPNO low-resolution spectra acquired in 1988 January were flux-calibrated using observations of standard stars. The other data have not been flux-calibrated in either relative or absolute terms.

The SPLOT software in IRAF was used to measure the strengths, central wavelengths, widths, and equivalent widths of the emission lines in galaxy spectra that had been spatially averaged along the slit to give adequate signal-to-noise. The $\mathrm{H} \alpha+[\mathrm{N}$ II] complex was deblended with the three lines constrained to have the appropriately fixed wavelength separations and the same widths. The [S II] doublet was similarly deblended, but the widths and wavelengths were not constrained relative to those of $\mathrm{H} \alpha+[\mathrm{N}$ II] .

\section{RESULTS}

\section{a) Kinematics}

As summarized in $\S \mathrm{I}$, the release of energy associated with a starburst in a circumnuclear molecular disk should drive a bipolar outflow along the rotation axis of the disk (CC; TI). The kinematic signature of such a "superwind" should be especially clear in cases in which the collimating disk is viewed edge-on. This suggests that edge-on galaxies that are strong 
TABLE 2

OBSERVATIONAL PARAMETERS

\begin{tabular}{|c|c|c|c|c|c|}
\hline $\begin{array}{l}\text { Telescope } \\
\text { (1) }\end{array}$ & $\begin{array}{c}\text { Instrument/Detector } \\
(2)\end{array}$ & $\begin{array}{l}\text { Slit Size } \\
\text { (3) }\end{array}$ & $\begin{array}{c}\text { Spectral } \\
\text { Range } \\
\text { (4) }\end{array}$ & $\begin{array}{l}\text { Resolution } \\
\text { (5) }\end{array}$ & $\begin{array}{l}\text { Dates } \\
(6)\end{array}$ \\
\hline CTIO 4 m ............. & $\begin{array}{l}\text { Cassegrain spectrograph } \\
\text { with GEC CCD }\end{array}$ & $2^{\prime \prime} \times 100^{\prime \prime}$ & $6100-6900 \AA$ & $3.5 \AA$ & 1987 Jan \\
\hline KPNO 4 m .......... & $\begin{array}{l}\text { RC spectrograph with } \\
\text { TI2 CCD }\end{array}$ & $2^{\prime \prime} \times 300^{\prime \prime}$ & $\begin{array}{l}6150-6900 \AA \\
6150-6900 \AA \\
6150-6900 \AA \\
6150-6900 \AA \\
4300-7100 \AA\end{array}$ & $\begin{array}{l}3.5 \AA \\
3.5 \AA \\
3.5 \AA \\
3.5 \AA \\
13.5 \AA\end{array}$ & $\begin{array}{l}\text { 1986 Nov } \\
\text { 1987 Apr } \\
\text { 1987 May } \\
\text { 1990 Jan } \\
\text { 1988 Jan }\end{array}$ \\
\hline
\end{tabular}

NOTES.-Key to cols. (4) and (5) follows:

Col. (4).-Approximate wavelength range covered in the rest frame of the galaxy observed.

Col. (5).-FWHM measured for bright night-sky lines.

far-infrared (FIR) sources are the ideal testing grounds for the superwind phenomenon. Fortuitously, the three FIR-brightest galaxies in the IRAS Point Source Catalog (and the three nearest FIRGs) are all disk galaxies viewed nearly edge-on: M82, NGC 253, and NGC 4945. For each galaxy we have therefore acquired spectra both along the optical minor axis and parallel to (but offset from) the major axis. Because the FIR-bright galaxy NGC 3079 is also viewed nearly edge-on and has been previously suggested as a superwind candidate (Ford et al. 1986; Irwin et al. 1987), we have obtained a similar pair of spectra of it.

In HAM, we suggested that the large and morphologically spectacular emission-line nebulae associated with Arp 220 and NGC 6240 were manifestations of superwinds in "ultraluminous" ( $\left.L_{\mathrm{IR}} \approx 10^{12} L_{\odot}\right)$ IR galaxies. We will present kinematic data to support this suggestion below.

\section{i) $M 82$}

Bland and Tully (1988) have recently summarized the long and complex history of investigations of the halo of this muchstudied FIRG/starburst galaxy (see Rieke et al. 1980), and have published Fabry-Perot data that clearly show that a largescale bipolar outflow (superwind) is occurring along the optical minor axis of the galaxy. The kinematic signature of the superwind-regions above and below the galaxy disk in which the emission lines are split into double-peaked profiles-was first noted by Axon and Taylor (1978). An example of such a double-peaked emission-line profile in M82 is shown in Figure 2. Since M82 is the prototype superwind galaxy, we will present and discuss our own data to serve as a basis for comparison with the similar data we will discuss below for other galaxies.

The velocity fields along the minor and offset-major axes of the M82 nebula are shown in Figure 3. The most striking kinematic features are the regions of split lines mentioned above. Along the minor axis, this region begins about $300 \mathrm{pc}$ from the nucleus and extends out to at least $1100 \mathrm{pc}$ on both sides (we adopt a distance of $3 \mathrm{Mpc}$ for M82, implying a scale of $14.5 \mathrm{pc}$ $\left.\operatorname{arcsec}^{-1}\right)$. The region is about $300-400 \mathrm{pc}$ across along the offset-major axis (slit positioned $760 \mathrm{pc}$ south of the nucleus) and is centered on-axis where the nebula is brightest.

The velocity separation between the two components of the split lines is typically $200-250 \mathrm{~km} \mathrm{~s}^{-1}$ and shows neither a systematic radial gradient nor any significant difference between the NW and SE sides of the nebula. Each component of the split lines is spectroscopically resolved with a typical FWHM of 100-200 $\mathrm{km} \mathrm{s}^{-1}$. Throughout the nebula, the component nearer the systemic velocity of $230 \mathrm{~km} \mathrm{~s}^{-1}$ (heliocentric) is about 2 or 3 times brighter than the other component.

The flux-weighted mean velocity of the gas changes by about $300 \mathrm{~km} \mathrm{~s}^{-1}$ along the minor axis, with the SE (NW) side blueshifted (redshifted) relative to the systemic velocity. The velocity field does not resemble a classic rotation curve. Instead, there is essentially no velocity gradient across the region within $300-400 \mathrm{pc}$ of the nucleus, while beyond these radii (i.e., within the region of split lines) the velocities diverge from the systemic velocity as the radius increases. There is no significant change in radial velocity along the offset-major-axis slit position.

To interpret these results, it will prove crucial to determine the orientation of the outflow axis of the gas with respect to the line of sight. The emission-line gas and starlight are both fainter and more heavily reddened on the NW than on the SE side ( see $\S$ III $c$ below). The NW side is then presumably viewed through the slightly tipped dusty disk of M82, and is therefore on the far side. This is consistent with an outflow interpretation of the velocity field along the minor axis.

\section{ii) $N G C 253$}

NGC 253 is almost a perfect twin to M82 in its IR luminosity, distance, radio continuum power and morphology, nuclear molecular disk, X-ray emission, and edge-on orientation (see Rieke et al. 1980; Antonucci and Ulvestad 1988; MHV). Ulrich (1978) first discovered that there is a region of split (double-peaked) emission lines just to the SE of the nucleus of NGC 253, and proposed that they arose in an outflowing wind. We show an example of a split line profile in Figure 2. Ulrich (1978) also discovered strongly noncircular motions in the ionized gas at larger radii, but these latter have been subsequently modeled by Pence (1981) as arising in ordinary disk gas responding to the barred potential in NGC 253. As we discussed in MHV, the morphology of the region of ionized gas along the SE minor axis of NGC 253 is strongly limb brightened, suggesting that the outflowing gas defines a hollow cylin- 

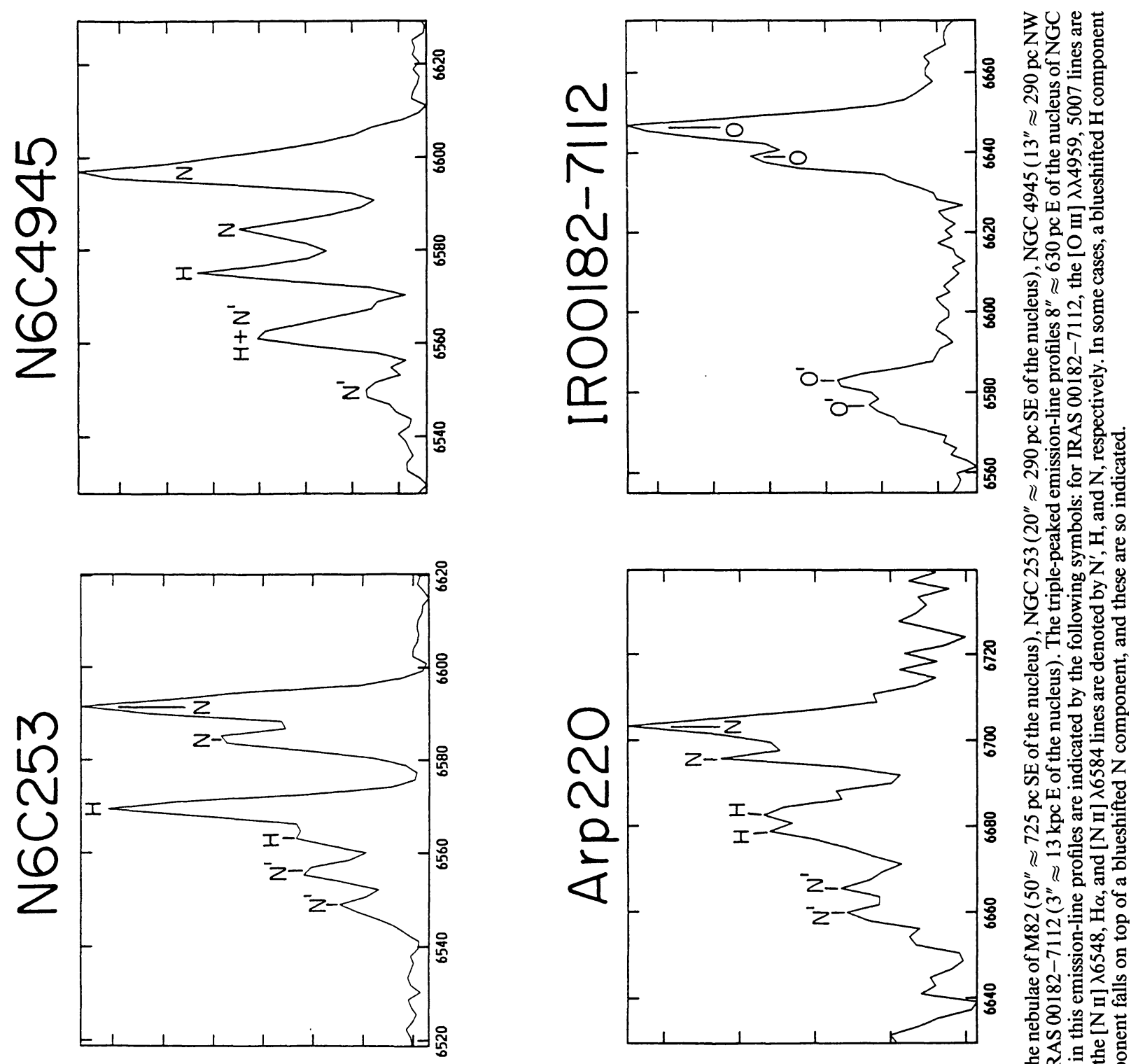

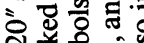

政

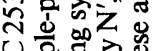

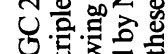

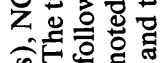

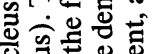

艺

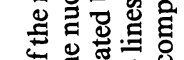

四

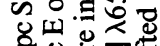

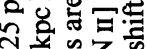

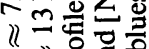

ㅇํㄴ 11 일

กิ่

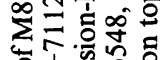

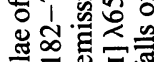

궁

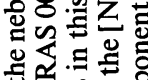

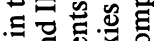
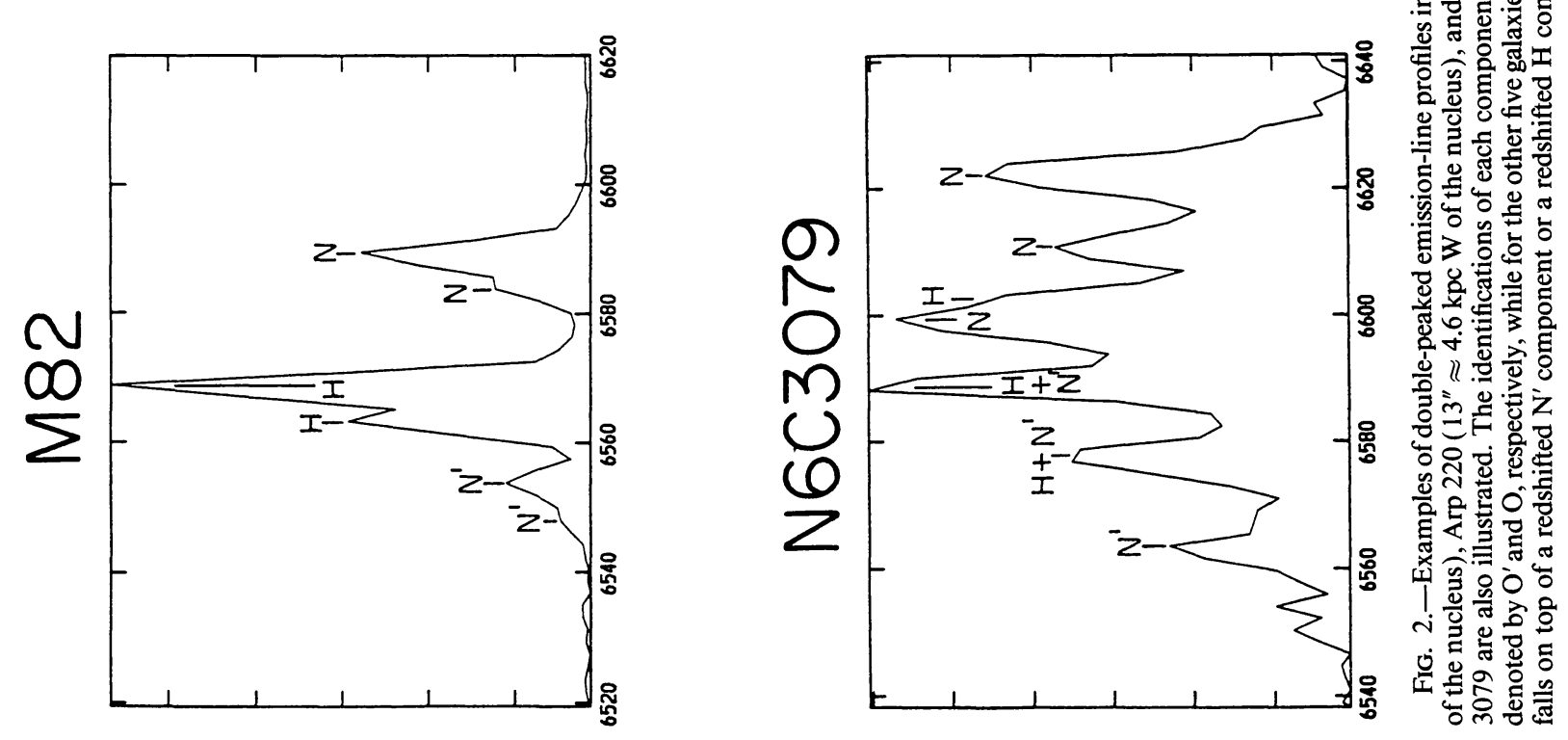


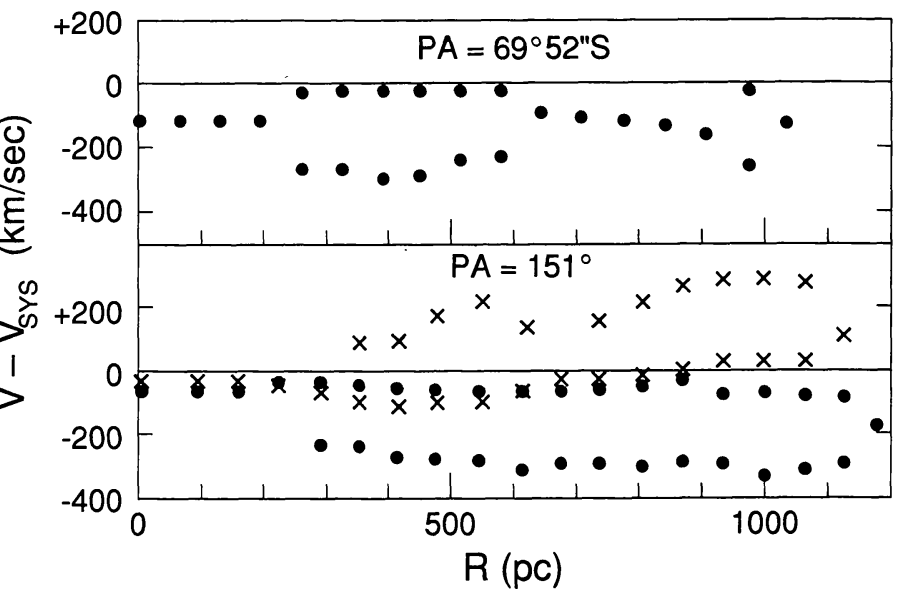

Fig. 3.-Velocity field in M82 as traced with the $\mathrm{H} \alpha$ and [N II] $\lambda \lambda 6548,6584$ emission lines. Velocities are given relative to an adopted systemic heliocentric velocity of $230 \mathrm{~km} \mathrm{~s}^{-1}$. Two position angles are shown. One passes through the center of the galaxy in P.A. $=151^{\circ}$ and is oriented along the minor axis of the galaxy. The velocities to the SE of the nucleus ( the near side of the galaxy disk) are indicated by solid points and the velocities to the NW (the far side) are indicated by crosses $(\times)$. The second position angle is oriented parallel to the major axis of the galaxy in P.A. $=69^{\circ}$, but is offset $52^{\prime \prime}(750 \mathrm{pc})$ to the south of the nucleus. For the second P.A., the data are plotted with NE to the left and SW to the right. Two velocities per position are given in the regions of double emission-line profiles.

der or conelike structure. Our new spectroscopic data not only confirm this limb brightening, but further support the superwind interpretation by revealing many striking kinematic similarities between the NGC 253 and M82 nebulae.

As seen in Figure 4, the region of split lines begins about 150 pc SE of the nucleus of NGC 253 and extends out an additional $500 \mathrm{pc}$ (we adopt a distance of 3 Mpc for NGC 253, implying a scale of $14.5 \mathrm{pc} \operatorname{arcsec}^{-1}$ ). Along the offset-major position angle (taken $290 \mathrm{pc} \mathrm{SE}$ of the nucleus) the region of split lines extends about $400 \mathrm{pc}$. No corresponding region of split lines is observed to the NW of the nucleus, presumably because it is on the far side of the slightly tipped dusty disk near the center of NGC 253 (see Fabbiano and Trinchieri 1984). Indeed, a region about $1 \mathrm{kpc}$ in extent to the north of the nucleus shows strong, redshifted centimeter wave $\mathrm{OH}$ emission which Turner (1985) interprets as outflowing material on the far side of the galaxy disk.

The velocity separation of the split lines visible optically ranges from about $250 \mathrm{~km} \mathrm{~s}^{-1}$ at positions nearest the nucleus to about $450 \mathrm{~km} \mathrm{~s}^{-1}$ at the SE-most extremity. The split lines consist of a weak blueshifted component (velocity ranging from $-200 \mathrm{~km} \mathrm{~s}^{-1}$ to nearly $-400 \mathrm{~km} \mathrm{~s}^{-1}$ relative to the adopted systemic velocity of $236 \mathrm{~km} \mathrm{~s}^{-1}$ ) and a redshifted component (velocity ranging from -50 to $+100 \mathrm{~km} \mathrm{~s}^{-1}$ relative to systemic) that is typically 2 or 3 times brighter. Each of the two components is about $150-250 \mathrm{~km} \mathrm{~s}^{-1}$ wide (FWHM).

The flux-weighted mean velocity of the gas to the SE along the minor axis is blueshifted by $50-100 \mathrm{~km} \mathrm{~s}^{-1}$ relative to the systemic velocity in the region within $300 \mathrm{pc}$ of the nucleus, but is slightly redshifted $\left(<50 \mathrm{~km} \mathrm{~s}^{-1}\right)$ at radii of $350-650 \mathrm{pc}$. Our slit position along the minor axis also intercepted two regions of high surface brightness with very narrow lines $(\ll 100$ $\mathrm{km} \mathrm{s}^{-1}$ FWHM) and with $\mathrm{H} \alpha$ much brighter than [N II] or [S II]. These are undoubtedly giant $\mathrm{H}$ II regions. They may be physically associated with the outflow or be ordinary disk $\mathrm{H} \mathrm{II}$ regions seen in projection along the minor axis. The radial velocities of both are within $20 \mathrm{~km} \mathrm{~s}^{-1}$ of systemic, as expected in this latter case. No systematic change in velocity greater than $100 \mathrm{~km} \mathrm{~s}^{-1}$ is detected over a distance of $600 \mathrm{pc}$ along the offset-major-axis position angle.

\section{iii) $N G C 4945$}

Even though NGC 4945 is the third brightest galaxy in the IRAS Point Source Catalog and the third closest FIRG (after M82 and NGC 253), it is considerably less well-studied than these two (see Brock et al. 1988 and Moorwood and Glass 1984 for recent investigations and summaries of the literature). At our adopted distance of $4.6 \mathrm{Mpc}$, both its IR and radio continuum luminosities are very similar to those of M82 and NGC 253. The galaxy is seen nearly edge-on, and the radio continuum arises in a nuclear region about $265 \times 90 \mathrm{pc}$ in size oriented along the galaxy major axis. The size of the nucleus at $10 \mu \mathrm{m}$ is about $135 \mathrm{pc}$, which is consistent with the upper limit of about $230 \mathrm{pc}$ to the size at $100 \mu \mathrm{m}$. This central radio/IR source is coincident with a region of strong molecular-line radiation (including $\mathrm{H}_{2} \mathrm{O}$ megamasers, Batchelor, Jauncey, and Whiteoak 1982). Whiteoak and Gardner (1979) first noted that the nuclear region was characterized by broad optical emission lines and line ratios that suggested shock heating or photoionization by an AGN. While Brock et al. (1988) therefore suggest that NGC 4945 is powered by a Seyfert nucleus, its many similarities to M82 and NGC 253 favor the starburst interpretation. As shown below, our new data reveal a further

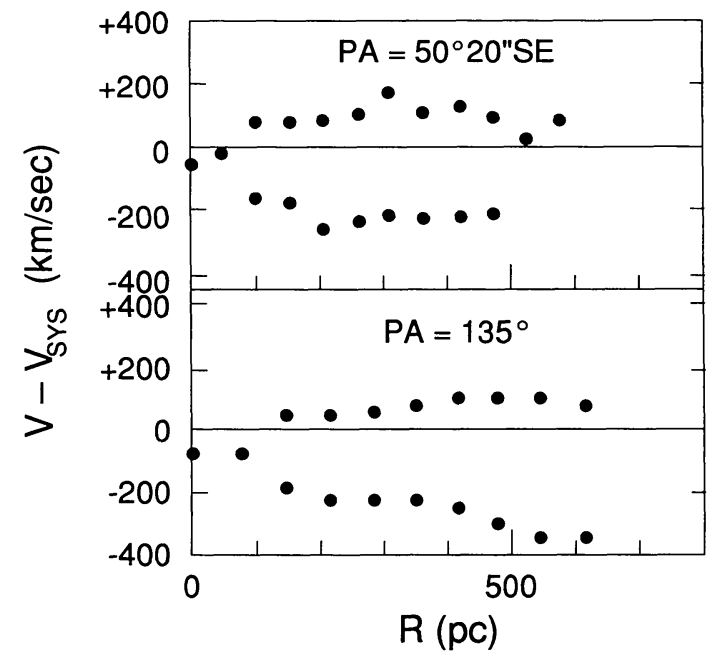

FIG. 4.-Velocity field in NGC 253 as traced with the $\mathrm{H} \alpha$ and [N II] emission lines. Velocities are given relative to an adopted systemic heliocentric velocity of $236 \mathrm{~km} \mathrm{~s}^{-1}$. The P.A. $=135^{\circ}$ spectrum passes through the nucleus and is oriented along the minor axis of the galaxy. The velocity field is given only to the SE of the nucleus (the gas to the NW is much fainter, presumably due to obscuration associated with its location on the back side of the disk; see text $)$. The P.A. $=50^{\circ}$ spectrum is oriented parallel to the major axis of the galaxy, but is offset $20^{\prime \prime}(290 \mathrm{pc})$ to the SE of the nucleus. For both spectra, the data are plotted with $\mathrm{E}$ to the left and $\mathrm{W}$ to the right. Two velocities per position are given in the regions of double emission-line profiles. 
striking similarity to M82 and NGC 253: ionized gas along the minor axis with strong line splitting (see Fig. 2 for an example of such a split-line profile in NGC 4945).

The velocity fields along the minor and offset-major axis of NGC 4945 are shown in Figure 5. To the NW, the region of split lines begins within $70 \mathrm{pc}$ of the nucleus and extends out to a distance of about $700 \mathrm{pc}$ (for our adopted scale of $22.3 \mathrm{pc}$ $\operatorname{arcsec}^{-1}$ ). The region to the SE of the nucleus is cut by a strong dust lane, and the emission lines and starlight are very faint there. If a region of split lines exists to the SE, it is evidently on the far side of the obscuring dust lane and is hence invisible. The region of split lines extends about $500 \mathrm{pc}$ along the offsetmajor-axis position angle, which was obtained $300 \mathrm{pc} \mathrm{NW}$ of the nucleus. Along both position angles, the surface brightness of the emission-line gas peaks at the outer edges of the region of split lines, suggesting that the gas defines a bubble-like structure. This kinematic structure is considerably smaller than, and apparently imbedded within, the $7 \times 1.5 \mathrm{kpc}$ "hollowcone" morphological feature visible to the NW of the nucleus in the broad-band image of NGC 4945 published by Naomasa (1990).

The velocity separation of the split lines ranges from about $300 \mathrm{~km} \mathrm{~s}^{-1}$ near the "walls" of the bubble to nearly $600 \mathrm{~km} \mathrm{~s}^{-1}$ near the center (this is particularly clear along the offset-major axis). The line profiles all consist of a bright component that is redshifted by $50 \pm 50 \mathrm{~km} \mathrm{~s}^{-1}$ relative to the systemic velocity of $563 \mathrm{~km} \mathrm{~s}^{-1}$ (heliocentric), and a component that ranges from 2-5 times fainter and is blueshifted relative to systemic by 250 $\mathrm{km} \mathrm{s}^{-1}$ to nearly $550 \mathrm{~km} \mathrm{~s}^{-1}$. Each component of the split lines has a FWHM of 200-400 $\mathrm{km} \mathrm{s}^{-1}$.

There are also four bright emission-line knots with $\mathrm{H} \alpha$ much brighter than [N II] or [S II] located out to $\approx 750 \mathrm{pc}$ (in

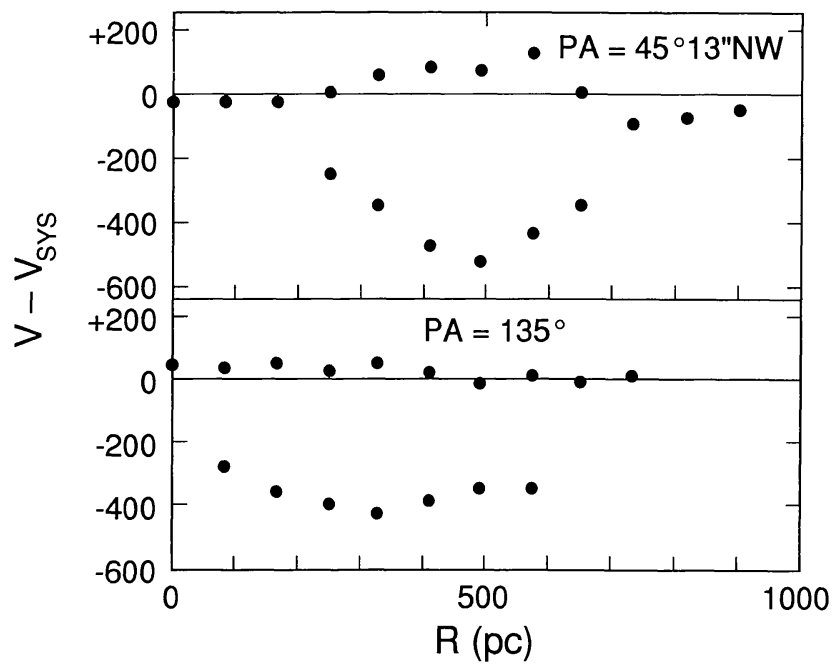

Fig. 5.--Velocity field in NGC 4945 as traced with the $\mathrm{H} \alpha$ and [N II] emission lines. Velocities are given relative to an adopted systemic heliocentric velocity of $563 \mathrm{~km} \mathrm{~s}^{-1}$. The P.A. $=135^{\circ}$ spectrum passes through the nucleus and is oriented along the minor axis of the galaxy. The velocity field is given only to the NW of the nucleus (the gas to the SE is very faint, presumably due to heavy obscuration associated with its location on the back side of the galaxy disk; see text). The spectrum in P.A. $=45^{\circ}$ is oriented parallel to the galaxy major axis, but is offset 13" (290 pc) to the NW of the nucleus. The data are plotted with $E$ to the left and $W$ to the right for both P.A.'s. Two velocities per position are given in the regions of double emission-line profiles. projection ) along the minor axis of NGC 4945. These are evidently giant $\mathrm{H}$ II regions, either associated with the outflow or located in the galaxy disk and seen along the minor axis only in projection. The velocities of the four $\mathrm{H}$ II regions range between $\pm 75 \mathrm{~km} \mathrm{~s}^{-1}$ of the galaxy's systemic velocity.

\section{iv) $N G C 3079$}

While the edge-on spiral galaxy NGC 3079 is similar in its IR luminosity to M82, NGC 253, and NGC 4945, it is 4-5 times more distant (we adopt $16.3 \mathrm{Mpc}$, yielding a scale of 79 $\mathrm{pc} \operatorname{arcsec}^{-1}$ ). Young, Claussen, and Scoville (1987) have discovered a bright, kiloparsec-scale molecular disk in the nucleus, and Lawrence et al. (1985) have shown that the central mid-IR source is spatially extended by at least $500 \mathrm{pc}$. The nucleus of NGC 3079 is also the site of the most powerful known $\mathrm{H}_{2} \mathrm{O}$ megamaser (Henkel et al. 1984). An emissionline structure having a "ruptured bubble" morphology and protruding out along the minor axis of the galaxy was reported by Ford et al. (1986). Irwin et al. (1987) have discovered a peculiar tail of H I associated with NGC 3079's companion galaxy, NGC 3073, and pointing directly away from NGC 3079 in projection. They have proposed that it is material that is being swept out of NGC 3073 by a superwind from NGC 3079. Since NGC 3073 is itself a starburst galaxy (aka Mrk 131), Irwin et al. (1987) speculate that the NGC 3079 wind may have helped initiate the starburst in NGC 3073.

Our spectroscopic data provide more support for a superwind in NGC 3079: as in the three galaxies discussed above, we find a region of split lines along the minor axis of NGC 3079 (see Figs. 2 and 6). However, in NGC 3079 the relative velocities are much larger, and the kinematics appear to be more complex. Along the minor axis, the region of split lines is located at distances of $200-850 \mathrm{pc}$ from the nucleus on the east side and 850-1250 pc from the nucleus on the west side. The lines and starlight are fainter to the west, and this is evidently on the far side of the galactic disk. The region of split lines also extends about $1200 \mathrm{pc}$ in the offset-major-axis spectrum, which was taken $555 \mathrm{pc}$ to the east of the nucleus. Comparing our spectra to the emission-line image published by Ford $e t$ al. (1986), it is clear that the region of split lines to the east corresponds to the limb-brightened bubble-like structure they discovered.

Along the minor axis, the split lines consist of three components of roughly equal strength, with each having a FWHM of $250-450 \mathrm{~km} \mathrm{~s}^{-1}$. One component has a velocity within $50 \mathrm{~km}$ $\mathrm{s}^{-1}$ of the systemic velocity of $1138 \mathrm{~km} \mathrm{~s}^{-1}$ (heliocentric), and the other two are redshifted and blueshifted, respectively, by typically $\pm 400-600 \mathrm{~km} \mathrm{~s}^{-1}$ relative to systemic. Along the offset-major-axis position angle, the kinematics are even more remarkable: over a region that extends from just north of the center of the eastern "bubble" to its northern edge $500 \mathrm{pc}$ away, a kinematic component that is blueshifted by nearly $1600 \mathrm{~km} \mathrm{~s}^{-1}$ relative to systemic is present. This region was just missed by the minor-axis slit position which passed to the south through the "bubble" center. To our knowledge, a velocity change of over $2000 \mathrm{~km} \mathrm{~s}^{-1}$ within a kiloparsec-scale nebula is unprecedented in astronomy. Velocities this large on scales this large can only arise in a violent outflow.

A deep $\mathrm{H} \alpha$ [N II] image of NGC 3079 shows faint nebulosity 


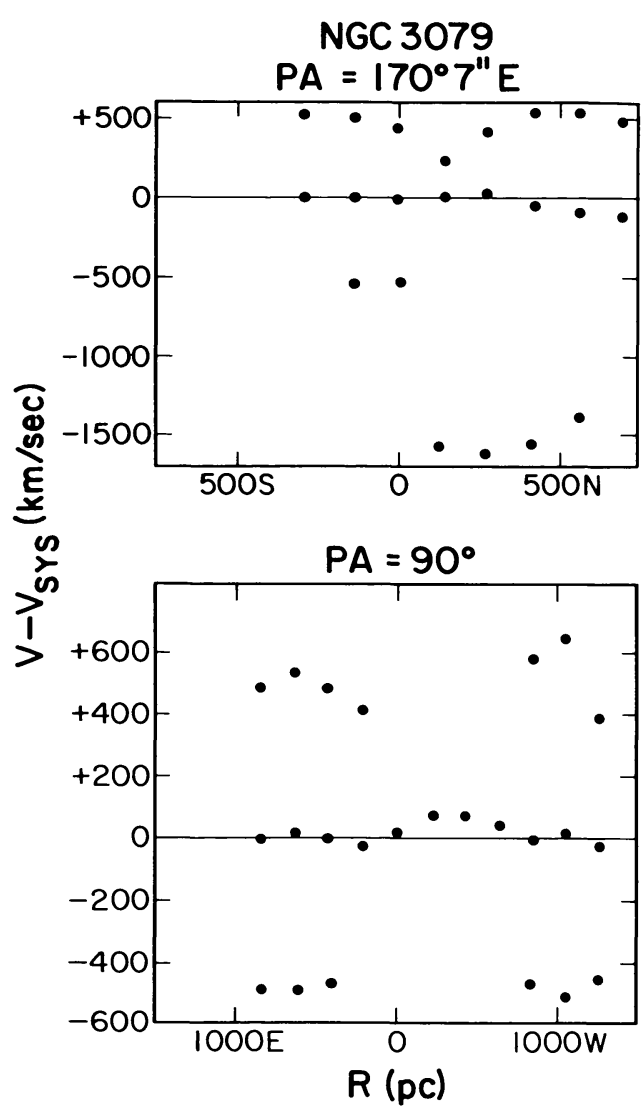

FIG. 6.-Velocity field in NGC 3079 as traced by the $\mathrm{H} \alpha$ and [N II] emission lines. Velocities are given relative to an adopted systemic heliocentric velocity of $1138 \mathrm{~km} \mathrm{~s}^{-1}$. The P.A. $=90^{\circ}$ spectrum passes through the nucleus and is oriented along the minor axis of the galaxy. Velocities are given on both sides of the nucleus ( $E$ to the left and $W$ to the right). The P.A. $=170^{\circ}$ spectrum is oriented parallel to the major axis of the galaxy, but is offset 7" $(550 \mathrm{pc})$ to the east of the nucleus ( $\mathrm{S}$ to the left and $\mathrm{N}$ to the right). Two or three velocities are given per position in the regions in which the emission-line profiles have two or three components, respectively (see text).

far out of the plane of NGC 3079 (Fig. 7, Pl. [00]), with an overall size of about $150^{\prime \prime} \approx 12 \mathrm{kpc}$. The nebulosity has a ragged X-shaped morphology formed by a system of lumpy filaments with individual lengths of 3-5 $\mathrm{kpc}$ and quasi-radial orientations. That is, the filaments do not extend directly into the nucleus, but rather seem to attach to the bright inner $(r \approx$ few kpc) disk of NGC 3079. We have obtained two spectra of this material (centered $7^{\prime \prime}=555 \mathrm{pc}$ and $20^{\prime \prime} \approx 1.6 \mathrm{kpc}$ east of the nucleus, both in P.A. $=170^{\circ}$ ). The gas has very orderly kinematics along the former slit position: the velocity changes smoothly from $+200 \mathrm{~km} \mathrm{~s}^{-1}$ to the south to $-150 \mathrm{~km} \mathrm{~s}^{-1}$ to the north (relative to systemic). The line emission along the outer $(1.6 \mathrm{kpc} \mathrm{E})$ slit position is exceedingly faint and exhibits a maximum velocity change of $\approx 300 \mathrm{~km} \mathrm{~s}^{-1}$. Line widths in the NGC 3079 filament system range from less than $100 \mathrm{~km} \mathrm{~s}^{-1}$ to $300 \mathrm{~km} \mathrm{~s}^{-1}$ FWHM, while the line ratios ([N II] $\approx \mathrm{H} \alpha$ and strong [ $\mathrm{S} \mathrm{II}]$ ) throughout this region imply that it is not simply gas photoionized by diffuse light from ordinary disk OB stars. We suggest that this material is ambient halo gas entrained into the boundary layers of a superwind. v) $\operatorname{Arp} 220$

Arp 220 is frequently cited as the prototype of the "ultraluminous" FIRGs (e.g., Sanders et al. 1988; Rieke et al. 1985), with a luminosity of about $10^{12} L_{\odot}$. Do these ultraluminous galaxies show any continuity in properties with the galaxies above? In HAM we reported the discovery of a gaseous nebula with a "double-bubble" shape and a size of $10 \times 24 \mathrm{kpc}$ associated with Arp 220, and speculated that this was material being swept up and accelerated outward by a superwind. Our new spectroscopic data support this idea, although the nebula has such a low surface brightness that our kinematic information is much less detailed than for the four galaxies discussed previously.

The kinematic evidence that the Arp 220 nebula is an expanding bubble is illustrated in Figure 8, which is a plot of the widths of the emission lines as a function of projected distance from the morphological center of the western (brighter) "bubble." Our spectra only marginally detect the gas projected onto/near the center of the fainter eastern "bubble." As seen in Figure 8, the line widths decline from about $700 \mathrm{~km} \mathrm{~s}^{-1}$ FWHM near the center of the bubble to only $300 \pm 100 \mathrm{~km} \mathrm{~s}^{-1}$ along the edges. This is-to first order-the kinematics expected of an expanding bubble (because of projection effects). The very broad emission lines near the bubble center are quite faint but appear to be double-peaked with a separation of about $450 \mathrm{~km} \mathrm{~s}^{-1}$ (Fig. 2).

The two spectra taken roughly along the axis of the "doublebubble" system (P.A. $=103^{\circ}$ and $135^{\circ}$ ) show only small changes in velocity, with the western bubble being blueshifted by about $100 \mathrm{~km} \mathrm{~s}^{-1}$ relative to the systemic velocity and the eastern bubble being redshifted by a similar amount. Note that the emission-line gas at the nuclear position is redshifted by about $100 \mathrm{~km} \mathrm{~s}^{-1}$ relative to the systemic velocity of $5400 \mathrm{~km}$ $\mathrm{s}^{-1}$ heliocentric (the latter coming from radio observations of the CO $J=1-0$ line; see Heckman et al. 1989 and Sanders and Mirabel 1985). The spectrum taken at P.A. $=0^{\circ}$ through the center of the western bubble shows no change in velocity greater than $100 \mathrm{~km} \mathrm{~s}^{-1}$. P. J. McCarthy (1989, private communication) has taken a spectrum in P.A. $=45^{\circ}$ along the central dust lane that bisects the "double-bubble" system and finds a velocity field that resembles a normal rotation curve with an amplitude (peak-to-peak) of about $300-350 \mathrm{~km} \mathrm{~s}^{-1}$.

To summarize, the kinematics of the ionized gas in Arp 220 are consistent with a two-component system: a rotating disk of gas and dust and an expanding bipolar nebula oriented roughly $40^{\circ}$ off the rotation axis of this disk. We note that Baan et al. (1987) have published VLA maps of gas that produces $21 \mathrm{~cm}$ $\mathrm{H}$ I absorption lines and $\mathrm{OH}$ megamaser emission lines in the central few hundred parsecs of Arp 220. This gas lies roughly along the inferred flow axis of the superwind at P.A. $=105^{\circ}$ and has one kinematic component that exhibits a velocity gradient of $\approx 100 \mathrm{~km} \mathrm{~s}^{-1}$ over $3^{\prime \prime}(1 \mathrm{kpc})$. As in the case of the much larger $\mathrm{H} \alpha$ "bubbles," the gas to the $\mathrm{E}(\mathrm{W})$ is redshifted (blueshifted) relative to systemic. While Baan et al. (1987) have interpreted the $\mathrm{H} \mathrm{I} / \mathrm{OH}$ structure as a rotating disk, it does not align with the optical dust lane, and we believe that it may instead be material associated with the inner part of the superwind. This interpretation is further supported by the additional high-velocity components of $\mathrm{H}$ I absorption and $\mathrm{OH}$ 
N

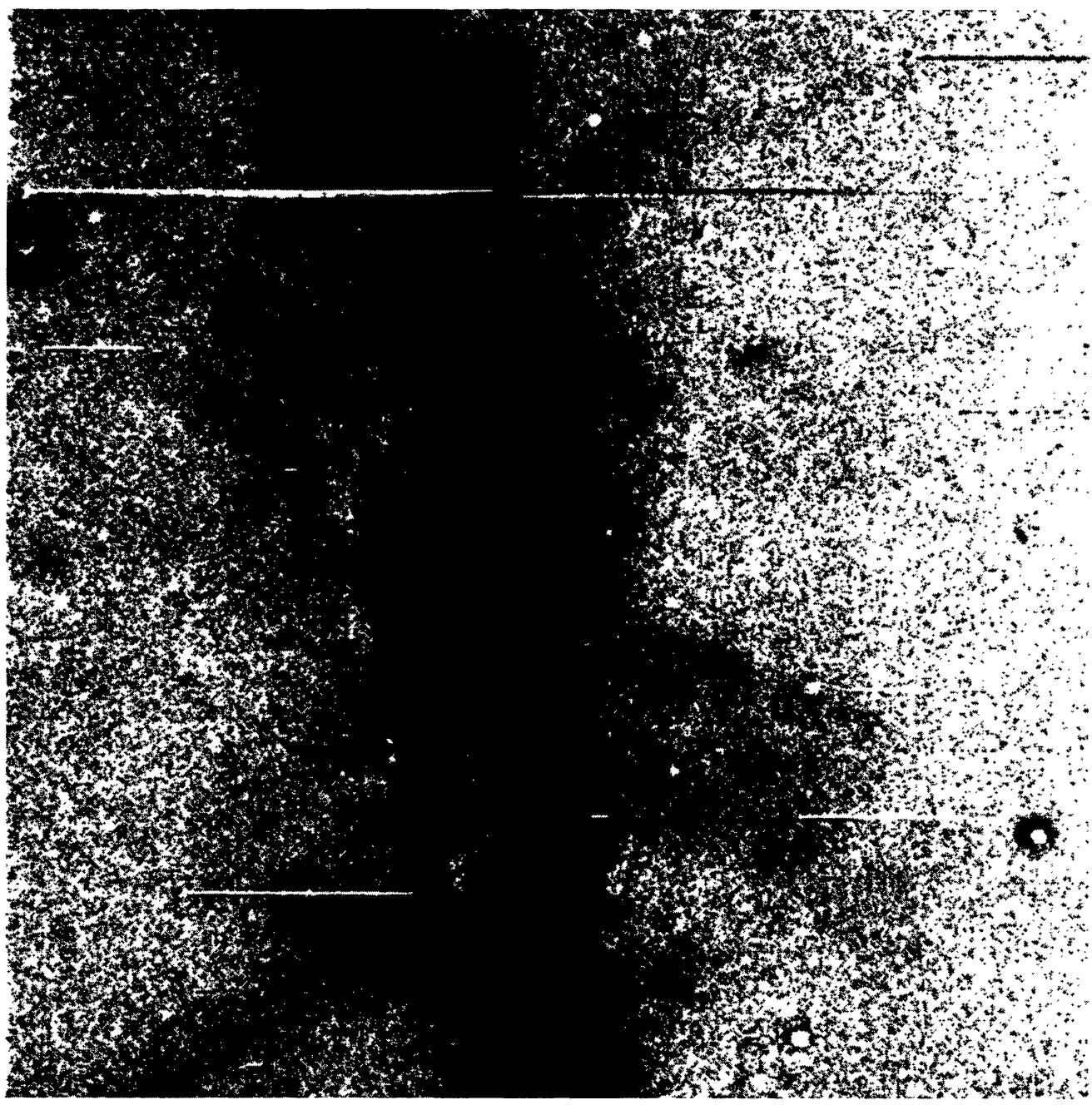

Fig. 7.-Gray-scale image of NGC 3079 in the light of the $\mathrm{H} \alpha[\mathrm{N} \mathrm{II}]$ lines. The imaged region is 150 arcsec across, and the image is displayed with north to the top and east to the right. Note the faint multiple plumes or loops extending far out of the disk of the galaxy.

emission along the superwind axis discovered by Baan et al. (1987).

\section{vi) $N G C 6240$}

NGC 6240 is similar to Arp 220 in distance and IR luminosity (see Rieke et al. 1985). While its optical-continuum morphology is complex and disturbed, the main body of the galaxy is quite flat $(a / b \approx 4: 1)$, and hence is likely to be a badly distorted disk viewed nearly edge-on. In HAM we presented an $\mathrm{H} \alpha[\mathrm{N}$ II $]$ image showing that NGC 6240 also has a large and morphologically spectacular nebula of ionized gas. This nebula consists of a bright hourglass-shaped region oriented at P.A. $=100^{\circ}$ along the minor axis of the galaxy disk with a size of about $10 \times 7 \mathrm{kpc}$ and a much fainter region 60 $\mathrm{kpc}$ across with multiple loops and filaments. As in the case of Arp 220, we speculated that the nebula might be a manifestation of a superwind. The "hourglass" bears a strong morphological resemblence to supercomputer simulations of superwinds in the "blow out" phase ( see TI; MMN).

We now present spectroscopic data that demonstrate that an internal velocity spread in excess of $1000 \mathrm{~km} \mathrm{~s}^{-1}$ is present in the NGC 6240 nebula, and that the highest velocities are seen along the inferred superwind axis. In Figure 9 we plot the spatial variation in line widths along the various position angles covered by our slit. Within a radius of about $4-5 \mathrm{kpc}$ from the nucleus the lines are extremely wide $\left(600-1200 \mathrm{~km} \mathrm{~s}^{-1}\right.$ FWHM). Comparison to the images in HAM demonstrates that the very broad lines are in fact found throughout the 


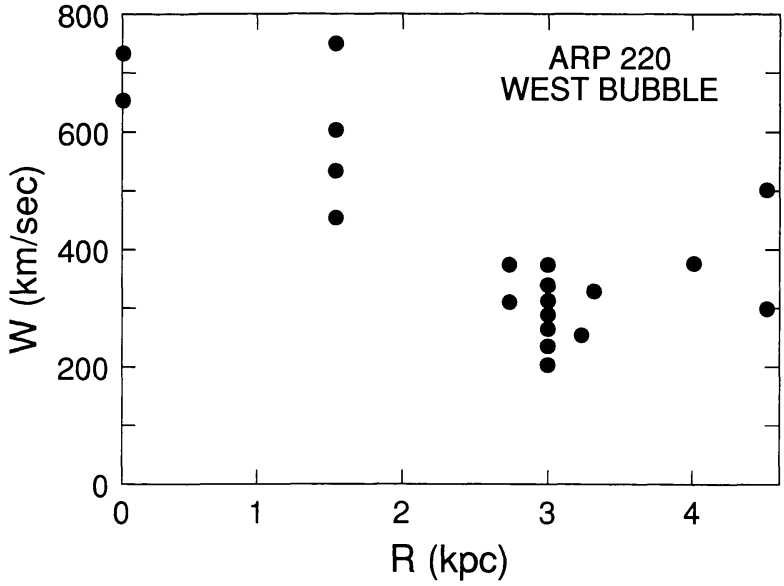

Fig. 8.-Widths (full width half-maximum) of the $\mathrm{H} \alpha$ and [N II] emission-lines as a function of projected distance from the center of the western (brighter) $\mathrm{H} \alpha$ "bubble" in Arp 220. The line profiles within a radius of about $1 \mathrm{kpc}$ from the bubble center are double-peaked with a splitting of about $450 \mathrm{~km} \mathrm{~s}^{-1}$ (see Fig. 2). The kinematics suggest that the $\mathrm{H} \alpha$ "bubble" is an expanding shell (see text).

"hourglass" feature. At larger radii the lines are narrower, though the gas is hardly quiescent (typically $200-600 \mathrm{~km} \mathrm{~s}^{-1}$ FWHM).

The velocity field is displayed in Figure 10. The kinematics is both complex and violent, with a full range of about 1000 $\mathrm{km} \mathrm{s}^{-1}$. Despite the complexity of the velocity field, one clear trend emerges (Fig. 11 and Table 3): the gas is most kinematically disturbed along the morphologically inferred superwind outflow axis (P.A. $=100^{\circ}$-see above $)$ and is least disturbed perpendicular to it.

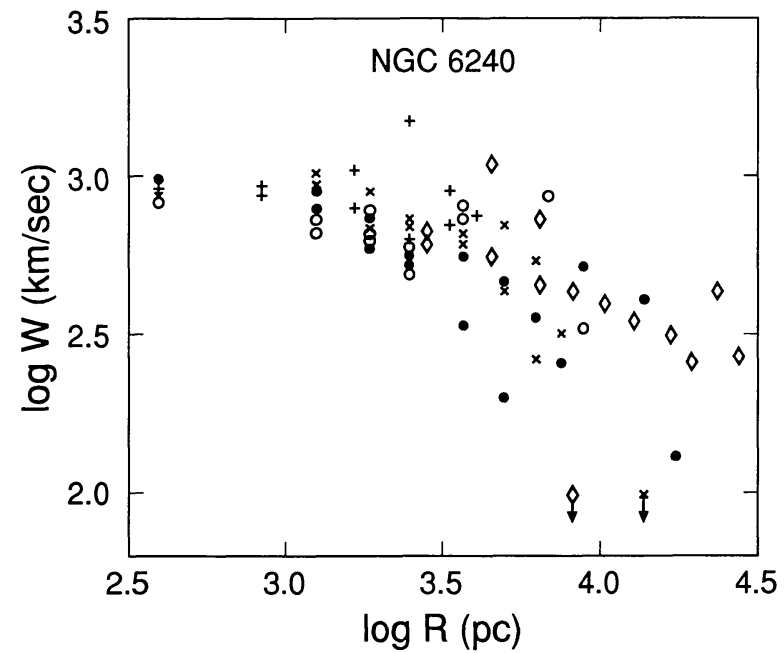

Fig. 9.-A log-log plot of line widths vs. projected distance from the nucleus for NGC 6240 . We have plotted the data from all the position angles covered by our spectra, with the following symbols: solid dots $\left(\right.$ P.A. $\left.=6^{\circ}\right)$, crosses $(\times)\left(\right.$ P.A. $\left.=71^{\circ}\right)$, diamonds $\left(\right.$ P.A. $=101^{\circ}$, slit offset $4^{\prime \prime} \mathrm{S}$ of the nucleus $)$, plus signs $(+)\left(\right.$ P.A. $\left.=109^{\circ}\right)$, and open circles $($ P.A. $=$ $\left.139^{\circ}\right)$. The region interior to $4-5 \mathrm{kpc}$ with the very broad lines corresponds to the "hourglass" feature discussed in the text.

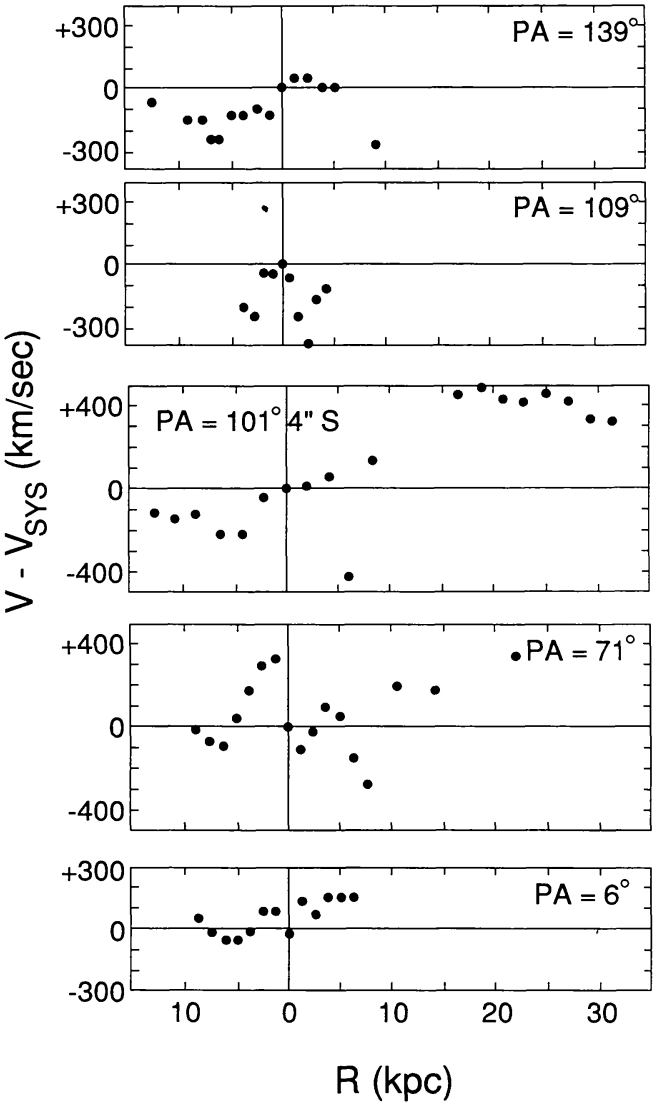

FIG. 10.-Velocity field in NGC 6240 as traced with the $\mathrm{H} \alpha$ and [N II] emission-lines along various position angles. All spectra passed through the nucleus except for that in P.A. $=101^{\circ}$, which passed $4^{\prime \prime} \mathrm{S}$ of the nucleus. In all cases, $\mathrm{E}$ is to the left and $\mathrm{W}$ to the right.

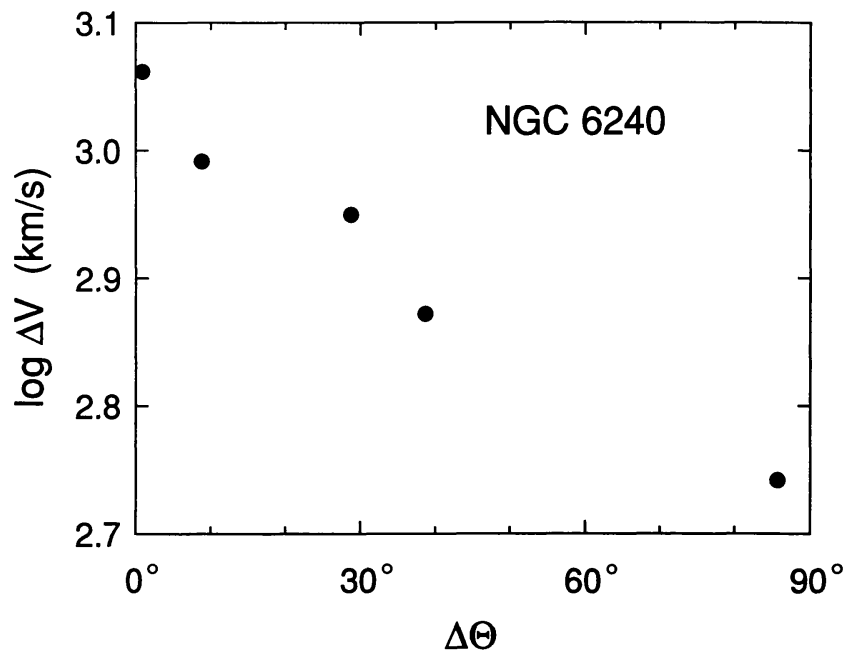

Fig. 11.-The log of the $\Delta v$ parameter (a measure of the amount of kinematic disturbance along a given position angle, defined to be the quadratic sum of the peak-to-peak velocity amplitude and the average FWHM of the line profiles along that P.A.) is plotted vs. the orientation of that P.A. relative to the outflow axis of the superwind in NGC 6240 (taken to be the symmetry axis of the "hourglass" and approximately perpendicular to the galaxy disk). 
TABLE 3

KINEMATICS OF THE NGC 6240 NEBULA

\begin{tabular}{cccccc}
\hline \hline P.A. & $\Delta \theta$ & $\sigma$ & $\Delta V_{\max }$ & $\langle w\rangle$ & $(4)$ \\
$(1)$ & $(2)$ & $(3)$ & 296 & 930 & $\begin{array}{c}\langle V\rangle \\
(6)\end{array}$ \\
\hline $101^{\circ} \ldots \ldots$. & $1^{\circ}$ & 123 & 400 & 690 & 1160 \\
$190 \ldots \ldots \ldots$. & $9^{\circ}$ & 178 & 620 & 880 & 970 \\
$71 \ldots \ldots .$. & $29^{\circ}$ & 107 & 350 & 630 & 884 \\
$139 \ldots \ldots .$. & $39^{\circ}$ & 80 & 220 & 660 & 750 \\
$6 \ldots \ldots .$. & $86^{\circ}$ & & 500 & 540 \\
\hline
\end{tabular}

NoTES.-Key to cols. (1)-(6) follows:

Col. (1).-Slit position angle ( $\mathrm{N}$ through $\mathrm{E})$.

Col. (2).-Relative angle between the slit and the adopted superwind outflow axis in P.A. $=100^{\circ}$ (the axis of symmetry of the "hourglass" feature and approximately perpendicular to the galaxy disk-see text and HAM).

Col. (3).- The rms variation of the line radial velocity centroid relative to systemic velocity along the specified position angle: $\sigma \equiv\left[N^{-1} \Sigma\left(v_{i}-v_{\text {sys }}\right)^{2}\right]^{1 / 2} \mathrm{~km} \mathrm{~s}^{-1}$, where each velocity $v_{i}$ is measured at $N$ independent positions along the slit.

Col. (4).-Peak-to-peak radial velocity variation $\left(\mathrm{km} \mathrm{s}^{-1}\right)$ along the specified slit position angle.

Col. (5).-Average line width (FWHM in $\mathrm{km} \mathrm{s}^{-1}$ ) at the $N$ independent positions along the slit.

Col. (6).-Parameter measuring the amount of kinematic disturbance (in $\mathrm{km} \mathrm{s}^{-1}$ ) associated with the specified slit position: $\langle v\rangle \equiv\left(\Delta V_{\max }^{2}+\langle w\rangle^{2}\right) 1 / 2$.

While the kinematics of the ionized gas in NGC 6240 cannot be as easily understood as in the galaxies discussed earlier, the correlation shown in Figure 11, the extremely broad lines within the $10 \mathrm{kpc}$-scale hourglass (Fig. 9), and the large velocity range present on even larger scales (Fig. 10) strongly suggest that an outflow is occurring. The kinematic disorganization evident in Figure 10 may reflect either an intrinsic dynamical complexity, or simply the result of viewing in projection a morphologically complex but dynamically simple outflow (e.g., a redshifted blob next to a blueshifted one may be a receding blob on the back side of the outflow projected next to an approaching blob on the near side). A more detailed exploration of the kinematics of the entire nebula would be most revealing.

Finally, we note the two very bright knots located about 8.5 $\mathrm{kpc}$ west and $3.5 \mathrm{kpc}$ WSW of the nucleus. These have line widths less than $100 \mathrm{~km} \mathrm{~s}^{-1} \mathrm{FWHM}$, and $\mathrm{H} \alpha$ is about 15 times brighter than [S II] $\lambda \lambda 6717+6731$ and at least 25 times brighter than [ $\mathrm{N}$ II] $\lambda 6584$. These knots are probably giant $\mathrm{H}$ II regions with $\mathrm{H} \alpha$ luminosities of about $4 \times 10^{39} \mathrm{ergs} \mathrm{s}^{-1}$ and $9 \times 10^{39}$ ergs $\mathbf{s}^{-1}$, respectively (uncorrected for internal extinction). Such luminosities are comparable to those of the most luminous known giant $\mathrm{H}$ II regions (typically found only in Sc I galaxies; see Kennicutt 1988). The weakness of the [N II] and [S II] lines suggest very low metal abundances (the models of McCall, Rybski, and Shields 1985 imply metalicities less than $20 \%$ solar). These two "super-jumbo" H II regions are especially intriguing because they lie (at least in projection) close to the inferred outflow axis of the superwind. Could these be regions of intense star formation triggered by the ram pressure of the outflow?

\section{vii) IRAS 00182-7112}

This galaxy at a redshift of 0.327 was the most luminous galaxy in the large sample of high-luminosity FIRGs investigated by Armus, Heckman, and Miley (1989). It is in fact comparable in luminosity to IRAS $09104+4109$, the "most luminous known infrared galaxy" (Kleinmann et al. 1988). IRAS 00182-7112 has a monochromatic power $\left(\lambda P_{\lambda}\right)$ at 45 $\mu \mathrm{m}$ (in its own rest frame) of $4.8 \times 10^{12} L_{\odot}$ versus $3.9 \times 10^{12}$ $L_{\odot}$ for IRAS $09104+4109$, but is less powerful at $18 \mu \mathrm{m}$ in its own rest frame $\left(\lambda P_{\lambda}=2.1 \times 10^{12} L_{\odot}\right.$ vs. $6.6 \times 10^{12} L_{\odot}$, respectively). Perhaps IRAS 00182-7112 should be awarded the title "most powerful far-infrared galaxy" and IRAS $09104+4109$ the title "most powerful mid-infrared galaxy!"
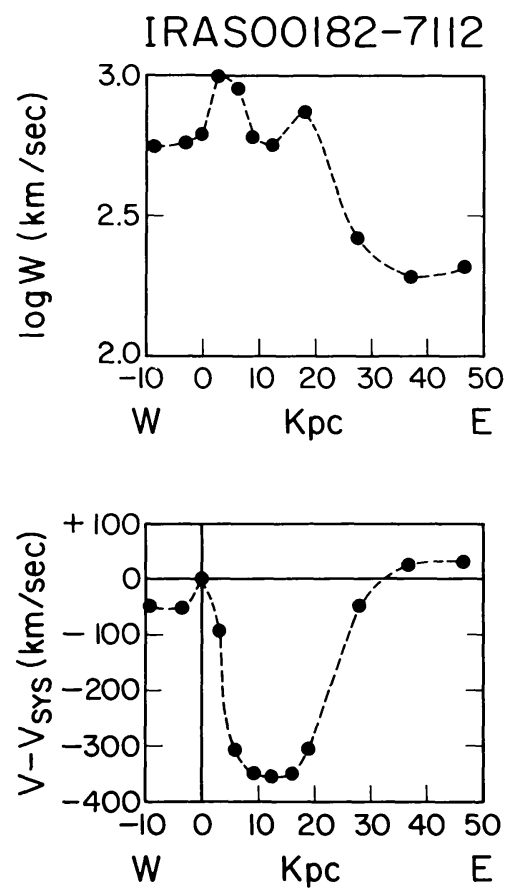

FIG. 12.-Kinematics of the gas in P.A. $=90^{\circ}$ in IRAS 00182-7112 as traced with the [O III] $\lambda 5007$ emission line. $(a)$ The radial velocity (with respect to the nucleus). ( $b$ ) The FWHM of the emission line. Note the very large line widths over a region tens of $\mathrm{kpc}$ in size. 
In any event, IRAS $00182-7112$ is a fascinating object. Our long-slit spectroscopy in P.A. $=90^{\circ}$ reveals bright ionized gas extending nearly $50 \mathrm{kpc}$ to the east and $10 \mathrm{kpc}$ to the west of the nucleus. The kinematics of this material is highly disturbed (see Fig. 12): line widths are in excess of $600 \mathrm{~km} \mathrm{~s}^{-1}$ (FWHM) over a region about $30 \mathrm{kpc}$ in diameter, with line widths reaching $1000 \mathrm{~km} \mathrm{~s}^{-1}$ in the region 3-7 kpc to the east of the nucleus. The region about $10 \mathrm{kpc}$ to the east exhibits double emission-line profiles with a velocity splitting of $300-400 \mathrm{~km} \mathrm{~s}^{-1}$ (see Fig. 2). The gas velocities are strongly blueshifted out to radii of about $25 \mathrm{kpc}$ to the east of the nucleus, with a maximum blueshift of $300-400 \mathrm{~km} \mathrm{~s}^{-1}$ in the region $5-20 \mathrm{kpc}$ to the east. The gas located beyond about $25 \mathrm{kpc}$ to the east is much more quiescent kinematically than the gas closer in, with line widths of only $\approx 200 \mathrm{~km} \mathrm{~s}^{-1}$ and velocities within $100 \mathrm{~km} \mathrm{~s}^{-1}$ of the nuclear velocity.

viii) Other Kinematic Evidence for Superwinds

While many of the galaxies above were chosen for detailed study based on their proximity and orientation (a stellar and/ or gas disk seen roughly edge-on), there is additional evidence of a statistical nature that suggests that mass outflows are commonplace in FIRGs. First, Mirabel and Sanders (1988) have discovered that the optical emission lines are systematically blueshifted with respect to the H I $21 \mathrm{~cm}$ line in FIRGs. Since the latter almost certainly measures the true systemic velocity of the FIRG, the optical emission is then typically blueshifted by about $100 \mathrm{~km} \mathrm{~s}^{-1}$ relative to the systemic velocity. Second, Armus, Heckman, and Miley (1989) have shown that the optical emission-line profiles from the central kiloparsec of FIRGs are often blue-asymmetric in shape, consisting of a narrow ( few hundred $\mathrm{km} \mathrm{s}^{-1}$ ) core plus a broad (greater than $1000 \mathrm{~km}$ $\mathrm{s}^{-1}$ ) blueshifted base. They find that such profiles are especially common in the FIRGs whose relative emission-line intensities are consistent with shockheating (i.e., the FIRGs with LINER spectra; see the discussion in $\S$ III $b$ below). Both these results can be easily understood as arising in outflows like those described above: a bipolar outflow along the rotation axis of a disk in which dust obscuration by the disk hides the back (redshifted) side of the flow.

There are also a handful of additional galaxies with ionized gas that is kinematically very similar to the gas in the FIRGs described above (see the recent summary by Taniguchi et al. 1988). The most kinematically convincing and well-studied examples of outflows are NGC 1365 (Phillips et al. 1983a), NGC 1569 (de Vaucouleurs, de Vaucouleurs, and Pence 1974), NGC 1705 (Meurer 1989), NGC 5506 (Wilson, Baldwin, and Ulvestad 1985), NGC 7582 (Morris et al. 1985), and Mrk 509 (Phillips et al. 1983b). NGC 1569 and NGC 1705 are starbursting dwarf galaxies, and the others are Seyfert galaxies in which the observed outflow is along the minor axis of a gaseous disk with a high rate of star formation (as deduced from optical spectroscopy and/or strong far-IR and millimeter-wave line emission).

\section{b) Physical Conditions in the Emission-Line Nebulae}

\section{i) Electron Temperatures}

Shock models imply that for shock speeds less than $250 \mathrm{~km}$ $\mathrm{s}^{-1}$, the optical emission-line gas is primarily collisionally ion- ized, with resulting temperatures in the $\mathrm{O}$ III zone of about $\log T \approx 4.4-4.5$. In faster shocks, the optical emission-line gas is primarily photoionized by EUV/soft X-rays produced upstream just behind the shock, yielding $\log T \approx 4.0-4.3$ in the $O$ III zone (Binette, Dopita, and Tuohy 1985, hereafter BDT). Models of gas photoionized by O stars (McCall, Rybski, and Shields 1985 ) predict $O$ III temperatures of $\log T \approx 3.6-$ 4.1 (with $T$ inversely correlating with metal abundance). In principle, then, the temperature measured by the [O III] $\lambda \lambda 4959+5007$ to $\lambda 4363$ flux ratio could provide a decisive discriminant between these alternative ionization sources for the FIRG nebulae. Unfortunately, the only nebulae in our sample in which the surface brightness in [O III] was sufficient for us to measure (or set meaningful upper limits to) the relative strength of the $\lambda 4363$ line were those of NGC 1222, NGC 1614, NGC 3690, Mrk 266, and Mrk 273. In Mrk 266 and NGC 3690 we measure $\log T \approx 4.3-4.7$ at various places in the nebulae, while we measure $\log T \approx 4.1$ in the high-ionization Seyfert-like nebula of Mrk 273, and can only set upper limits of $\log T<4.4$ for NGC 1222 and NGC 1614. These results are not truly conclusive, but are consistent with collisional ionization by shocks in Mrk 266 and NGC 3690 and photoionization in the other three nebulae.

\section{ii) Electron Densities and Estimated Gas Pressures}

We have measured the spatial variation in the flux ratio of the [S II] $\lambda \lambda 6717,6731$ emission-line doublet for 12 FIRGs in the sample of Armus, Heckman, and Miley $(1989,1990)$ with nebulae of high surface brightness and large angular size: M82, NGC 253, NGC 1222, NGC 1614, NGC 3079, NGC 3256, NGC 3690, NGC 4194, NGC 4945, NGC 6240, Mrk 266, and Mrk 273. This list includes all the galaxies whose kinematic properties were discussed above, except for Arp 220 and IRAS 00182-7112, in which the off-nuclear gas is too faint.

The measured [ $\mathrm{S}$ II ] flux ratios and the atomic parameters of Canto et al. (1980) were used to determine electron densities in the standard way assuming that $T_{e} \approx 10,000 \mathrm{~K}$. We have plotted the resulting radial variation in electron density for each of the 12 FIRGs in Figure 13. In both shock-heated gas and gas photoionized by an energetic continuum, the region where the [S II] lines are produced has a thermal pressure of about $4 \times 10^{-12} n_{e}$ dynes $\mathrm{cm}^{-2}$ (e.g., Shull and McKee 1979; Kraemer 1986). This is equivalent to $T_{e}=10,000 \mathrm{~K}$ and $n_{\text {tot }} \approx$ $3 n_{e}$ to account for the fact that the $\mathrm{S}$ II exists in a partially neutral zone. We have therefore used this conversion factor to calculate the radial variations in pressure also indicated in Figure 13.

There are several principal conclusions to be drawn from our data and (as we will discuss in $\S$ IV below) the superwind model naturally accounts for these results both qualitatively and quantitatively:

1. Typical central densities are $500-1000 \mathrm{~cm}^{-3}$, corresponding to pressures of $2-4 \times 10^{-9}$ dynes $\mathrm{cm}^{-2}$. This pressure is in excellent agreement with the pressure inferred for the $\mathrm{X}$-ray-emitting gas in M82 (Schaaf et al. 1989). These densities and implied pressures are very high compared to those in normal interstellar media. For example, the thermal gas pressure in the local ISM in the Milky Way is about four orders of magnitude lower than the estimated central pressures in the FIRGs (e.g., McKee and Ostriker 1977). 


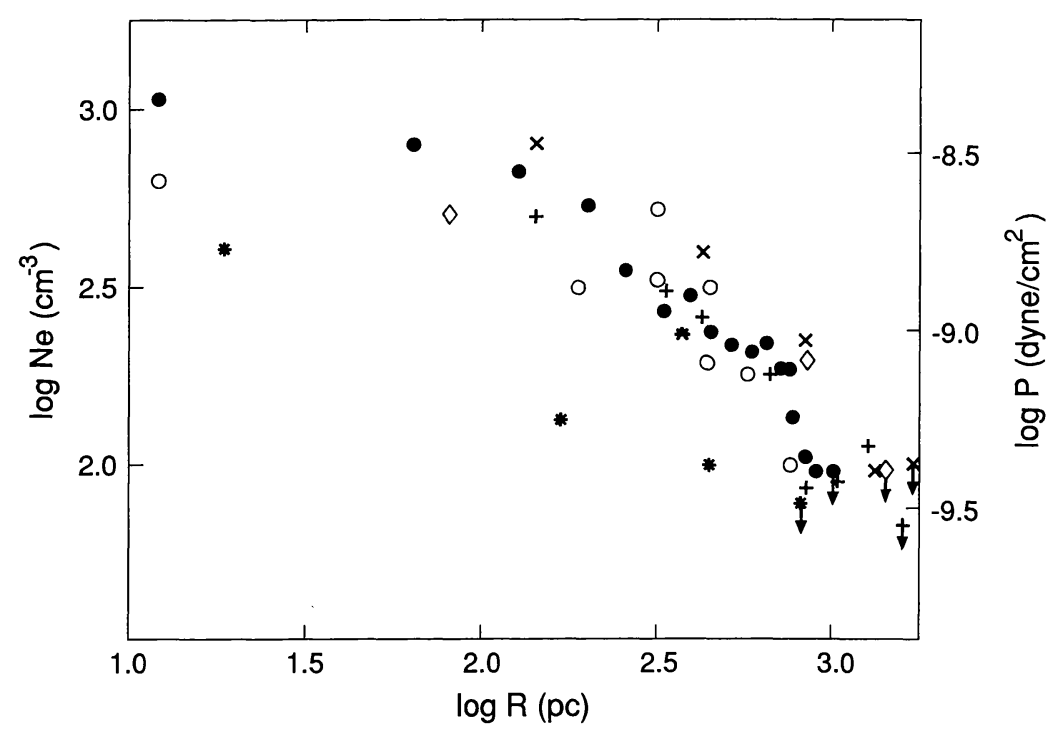

FIG. $13 a$

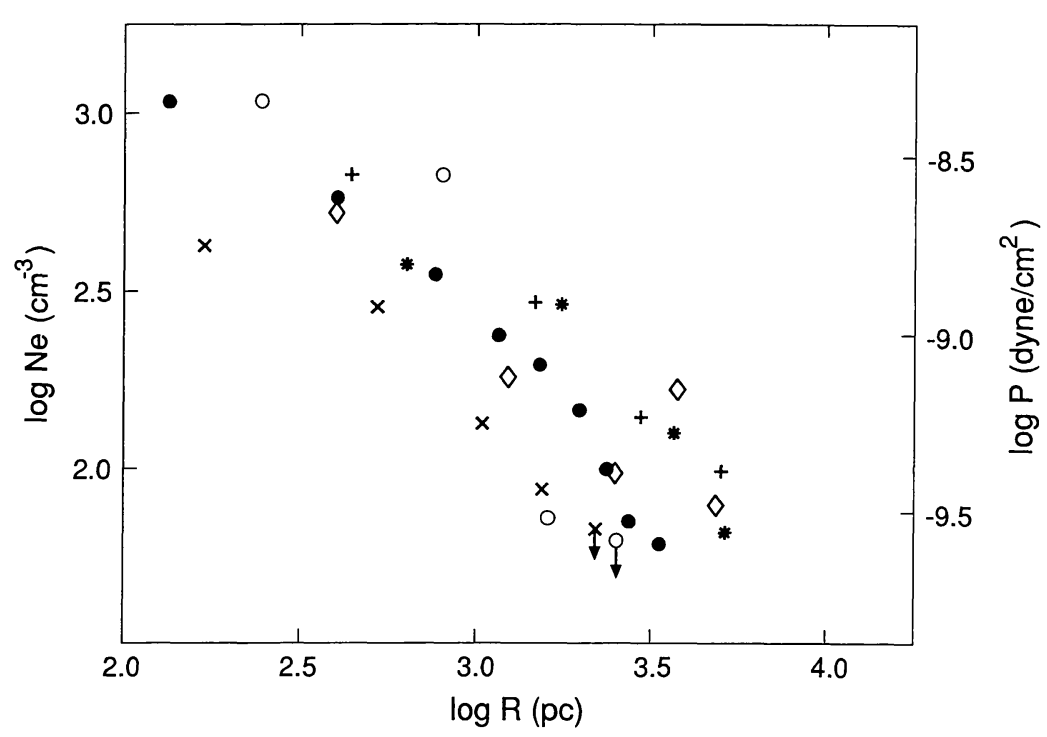

Fig. $13 b$

FIG. 13.-Log-log plots of electron density $\left(n_{e}\right)$ vs. projected distance from the nucleus. $(a)$ Galaxies with low IR luminosities $\left(<10^{11} L\right.$. $)$ are plotted with the following symbols: NGC 253 (open circles), NGC 1222 (plus signs), M82 (filled circles), NGC 3079 (diamonds), NGC 4194 (crosses), NGC 4945 (asterisks). (b) Galaxies with high IR luminosities $\left(>10^{11} \mathrm{~L}\right.$.) are plotted with the following symbols: NGC 1614 (open circles), NGC 3256 ( filled circles), NGC 3690 (crosses), NGC 6240 (diamonds), Mrk 266 (plus signs), and Mrk 273 (asterisks). Note that the plotted radial scale is shifted by one dex for the high luminosity galaxies. The plotted values of $n_{e}$ are averages of data on both sides of the nucleus and along different position angles (if available). The radius corresponding to the centralmost point is the radius-enclosing half of the surface area covered by the nuclear spectrum. The approximate gas pressures corresponding to the measured densities are indicated on the right-hand vertical axis. These assume that $T\left(n_{e}+n_{i}+n_{0}\right) / n_{e} \approx 30,000 \mathrm{~K}$, where $n_{i}$ and $n_{0}$ are the ion and neutral particle densities, respectively ( see text).

2. The densities/pressures decline systematically with radius. In the cases with the best data over the largest logarithmic range in radii (e.g., M82 and NGC 3256), the radial density/ pressure profile is shallow at small radii (less than a few hundred parsecs), but steepens at larger radii. The density reaches $100 \mathrm{~cm}^{-3}$ (approximately the low-density limit of the [S II] doublet) at radii ranging from several hundred to several thousand parsecs in the different FIRGs.

3. The galaxies with the higher IR luminosities (NGC 1614, NGC 3256, NGC 3690, NGC 6240, Mrk 266, and Mrk 273) have larger isobaric length scales than the galaxies with lower IR luminosities (M82, NGC 253, NGC 1222, NGC 3079, NGC 4194, and NGC 4945). The former galaxies all have IR luminosities of $10^{11}$ to $10^{12} L_{\odot}$, and the electron density reaches $100 \mathrm{~cm}^{-3}$ at radii of several kpc. The latter galaxies have IR luminosities of only few $\times 10^{10} L_{\odot}$, and the electron densities reach $100 \mathrm{~cm}^{-3}$ at radii of about a kpc.

4. For NGC 253, M82, NGC 3079, and NGC 4945 we have measured the lateral density profile along the offset-majoraxis slit positions discussed in $\S$ III $a$ above. We find that the 
densities measured perpendicular to the axis of the outflow follow the same $n_{e}(r)$ relation as the densities measured along the outflow axis (where $r$ is the projected distance to the $n u$ cleus). In particular, the densities and inferred pressures remain high $\left(n_{e} \approx 100-300 \mathrm{~cm}^{-3}\right.$ and $P \approx 4 \times 10^{-10}$ to $10^{-9}$ dynes $\left.\mathrm{cm}^{-2}\right)$ over regions whose lateral extents $(\approx 1-2 \mathrm{kpc})$ are at least 2 to 3 times larger than lateral extents of the regions with "split lines" described in $\S$ III $a$ above (see Table 4).

\section{iii) Other Emission-Line Ratios}

We have shown in MHV, HAM, and Armus, Heckman, and Miley (1989) that the relative strengths of the emission lines in the nuclei of FIRGs often require the presence of an ionization source for the gas different from, or in addition to, normal OB stars (about half the nuclei are LINERs, and the others have spectra similar to normal $\mathrm{H}$ II regions). Because the emissionline spectra of LINERs (and hence of many FIRGs) can be reproduced by models of shock-heated gas, we have suggested in the three above papers that this ionization source might be shocks driven into clouds accelerated outward by a superwind.

Here we report that the same argument applies even more strongly to the relative emission-line strengths in the extranuclear gas whose dynamical and physical properties we have described above. Indeed, AHM have discussed the prototypical case of M82 where the line ratios change from those of giant $\mathrm{H}$ II regions in the nucleus to those of a LINER at radii beyond about $300 \mathrm{pc}$ along the minor axis. We have plotted the flux ratio of the [O I] $\lambda 6300 / \mathrm{H} \alpha$ lines versus the flux-ratio of the [S II] $\lambda 6717+6731 / \mathrm{H} \alpha$ lines (Fig. 14) for the outer parts of the spatially extended nebulae discussed above. By "outer" we mean near the radius where $n_{e} \approx 100 \mathrm{~cm}^{-3}$, or at radii beyond $1 \mathrm{kpc}$ for Arp 220 and beyond $5 \mathrm{kpc}$ in IRAS 00182-7112. We have also indicated in Figure 14 the upper-rightmost part of the locus of normal giant $\mathrm{H}$ II regions (gas photoionized by normal OB stars), and the loci of old supernova remnants and Herbig-Haro objects (gas heated by shocks) and of models of shock-heated gas (Shull and McKee 1979; BDT). It is clear that the nebulae resemble shock-heated gas (and hence

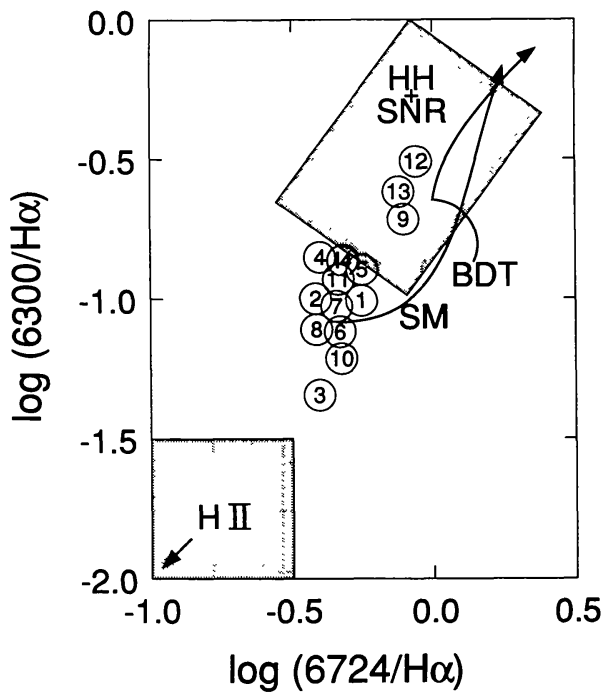

FIG. 14.-Plot of the log of the flux ratio of the [O I] $\lambda 6300$ and $\mathrm{H} \alpha$ emission lines vs. the log of the flux ratio of the [S II] $\lambda \lambda 6716,6731$ and $\mathrm{H} \alpha$ emission lines for the galaxies discussed in this paper. The plotted points correspond to averages of extranuclear regions only (see the text for details), and are given according to the following key: (1) NGC 253, (2) NGC 1222, (3) NGC 1614, (4) M82, (5) NGC 3079, (6) NGC 3256, (7) NGC 3690, (8) NGC 4194, (9) NGC 4945, (10) Mrk 266, (11) Mrk 273, (12) Arp 220, (13) NGC 6240, and (14) IRAS 00182-7112. We have also plotted the upper rightmost corner of the locus of giant $\mathrm{H}$ II regions (denoted " $\mathrm{H}$ II") and the locus of supernova remnants and Herbig-Haro objects (box denoted "HH+SNR"). The predictions of two sets of models of shock-heated gas (Shull and McKee 1979; Binette, Dopita, and Tuohy 1985) are plotted as arrows in the direction of increasing shock velocity (SM from 80-130 $\mathrm{km} \mathrm{s}^{-1}$; BDT from 86-167 $\mathrm{km} \mathrm{s}^{-1}$ ).

LINERs) far more strongly than they resemble the giant $\mathrm{H}$ II regions. We have measured the flux ratio of the [O III] $\lambda 5007 / \mathrm{H} \beta$ lines for nine of our nebulae (IRAS 00182-7112, NGC 1222, NGC 1614, M82, NGC 3690, NGC 4194, NGC 6240, Mrk 266, and Mrk 273). Except for Mrk 273, this ratio is of order unity, requiring shock velocities greater than $80 \mathrm{~km}$

TABLE 4

Miscellaneous Properties of THE M82, NGC 253, NGC 3079, aND NGC 4945 NebulaE

\begin{tabular}{cccccc}
\hline \hline $\begin{array}{c}\text { Galaxy } \\
(1)\end{array}$ & $\begin{array}{c}D_{\text {nuc }} \\
(2)\end{array}$ & $\begin{array}{c}D_{\text {sl }} \\
(3)\end{array}$ & $\begin{array}{c}D_{P} \\
(4)\end{array}$ & $\begin{array}{c}D_{L} \\
(5)\end{array}$ & $\begin{array}{c}\Omega_{w} / 4 \pi \\
(6)\end{array}$ \\
\hline M82 $\ldots \ldots \ldots \ldots \ldots$. & 750 & 400 & 1300 & 2500 & $>0.25$ \\
NGC $253 \ldots \ldots \ldots .$. & 290 & 500 & 800 & 1500 & $>0.4$ \\
NGC $3079 \ldots \ldots . .$. & 550 & 1000 & 2000 & $\approx 10000$ & $>0.5$ \\
NGC $4945 \ldots \ldots . .$. & 300 & 500 & 800 & 1000 & $>0.4$ \\
\hline
\end{tabular}

NoTES.-Key to cols. (2)-(6) follows:

Col. (2).-Distance by which the position of the long-slit spectrum taken parallel to the galaxy major axis was offset from the galaxy's nucleus (in pc).

Col. (3).- Transverse diameter (as measured along the slit position specified in col. [2]) of the region exhibiting "split" emission-line profiles).

Col. (4). - Transverse diameter of the region (as measured along the slit position specified in col. [2]) for which the electron density and gas pressure could be determined using the [S II] lines.

Col. (5).- Transverse diameter (as measured along the slit position specified in col. [2]) of the region with a LINER-type emission-line spectrum (strong [O I], [N II], and [S n] relative to $\mathrm{H} \alpha$ ).

Col. (6).- Minimum fraction of the full $4 \pi \mathrm{Sr}$ into which the wind must be flowing, based on the transverse extent of the region with measurable (high) pressures: $\Omega_{w} / 4 \pi=(1-\cos \theta)$, where $\theta=$ $\arctan \left(D_{P} / 2 D_{\text {nuc }}\right)$. 
$\mathrm{s}^{-1}$ for a good match to the models. Such velocities are also consistent with the other line ratios as well. The high-ionization spectrum of the Mrk 273 nebula (with [O III]/ $\mathrm{H} \beta$ about 10 ) is not well-matched by shock models. Its spectrum strongly resembles that of a type 2 Seyfert nucleus (Mrk 273 is itself classified as a type 2 Seyfert galaxy; see Koski 1978).

We also have discovered that the physical state of the extranuclear gas (as measured by the relative emission-line strengths) is related to the dynamical state of the gas: for the regions in the nebulae where the lines are broader than $300 \mathrm{~km}$ $\mathrm{s}^{-1}$ (FWHM), the [O I] line is typically $10 \%-50 \%$ as strong as $\mathrm{H} \alpha$, while for the regions where the line widths are less than $300 \mathrm{~km} \mathrm{~s}^{-1}$, the [O I] line is typically only $3 \%-10 \%$ as strong as $\mathrm{H} \alpha$. For the [S II] doublet, the corresponding values are $50 \%-$ $120 \%$ of $\mathrm{H} \alpha$ in the "broad-lined" regions and $25 \%-50 \%$ of $\mathrm{H} \alpha$ in the "narrow-lined" regions (Figs. 15a, 15b). Note, however, that even the kinematically quiescent gas has relative line ratios closer to those expected in shock-heated gas than in H II regions. Indeed, the LINER-type spectrum of the gas above and below the galactic disks in M82, NGC 253, NGC 3079, and NGC 4945 extends well beyond the regions with the split emission-line profiles ( see Table 4).

\section{c) Miscellaneous Global Properties of the M82 Nebula}

Because M82 is the FIRG/superwind for which the best set of data exists, it is worthwhile to summarize the important global properties of its nebula that can be derived from our data.

\section{i) Extinction}

We have measured the $\mathrm{H} \alpha / \mathrm{H} \beta$ flux ratio along the minor axis of M82 which-together with an assumption of an intrinsic case B ratio of 2.87 and a standard interstellar extinction curve-allows us to measure the extinction representative of the nebula. This information is summarized in Figure 16. The

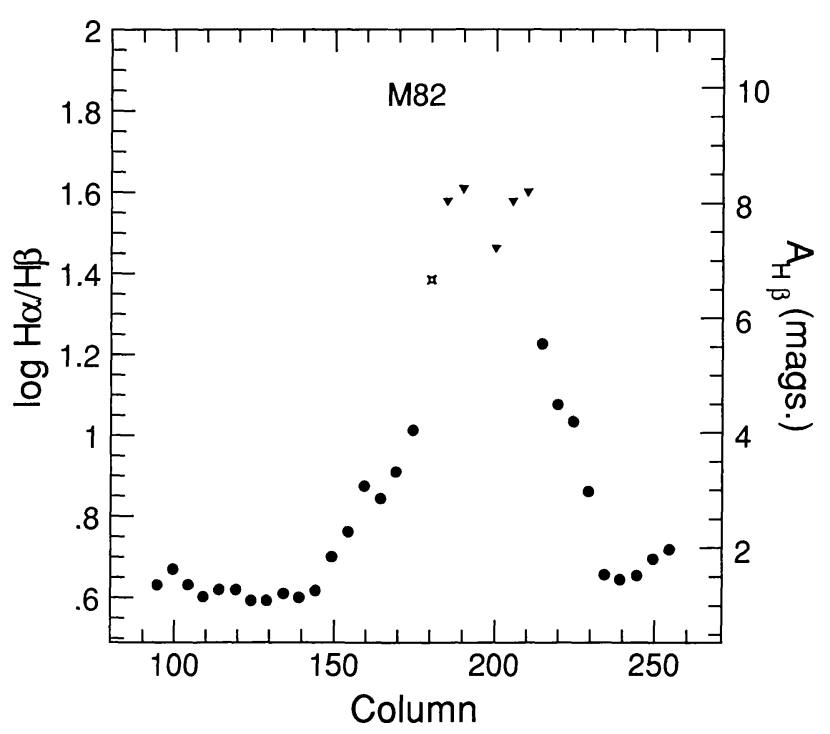

Fig. 16.-Plot of the $\log$ of the $(\mathrm{H} \alpha / \mathrm{H} \beta)$ flux ratio along the minor axis of M82 (P.A. $\left.=155^{\circ}\right)$. The horizontal axis is in pixels along the slit ( 1 pixel $\left.=0.89^{\prime \prime} \approx 13 \mathrm{pc}\right)$. The scale on the right-hand vertical axis gives the extinction in magnitudes at the wavelength of $\mathrm{H} \beta\left(A_{\mathrm{H} \beta}\right)$ implied by the measured flux ratio assuming an intrinsic ratio of 2.87 (case B with $T=$ $10,000 \mathrm{~K}$ ) and a normal interstellar extinction curve. Note the heavy extinction near the nucleus (nuclear position denoted by a star), the excess extinction to the NW compared to the SE, and the relatively small and constant extinction beyond a radius of about $300 \mathrm{pc}$ to the SE (column numbers less than 150).

$\mathrm{H} \alpha / \mathrm{H} \beta$ ratio remains roughly constant at about 4 to the SE from a distance of about $300 \mathrm{pc}$ to $1200 \mathrm{pc}$ from the nucleus. This corresponds to $A_{V} \approx 0.8$, or extinction by a factor of about 1.8 at $\mathrm{H} \alpha$. The ratio then rises steeply to values in excess of 10 near the nucleus, implying $A_{V}>3$ there. It remains high out to a radius of about $600 \mathrm{pc}$ to the NW of the nucleus before

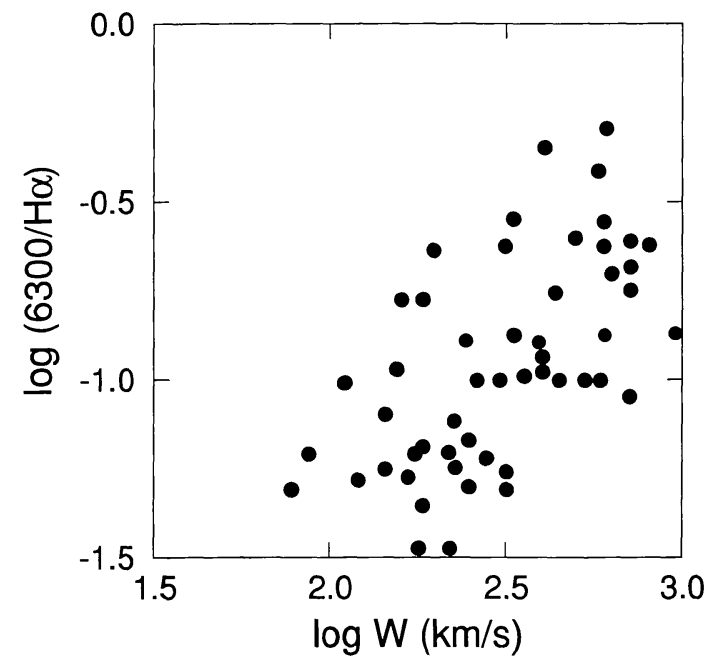

FIG. $15 a$

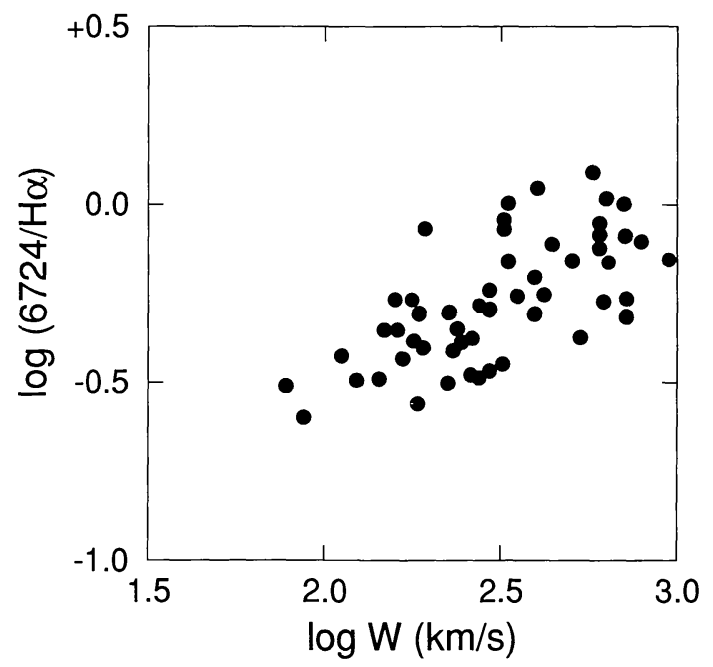

FIG. $15 b$

FIG. 15.-Plots of the $\log$ of the $(a)\left[\mathrm{O}_{\mathrm{I}}\right] \lambda 6300 / \mathrm{H} \alpha$ and $(b)\left[\mathrm{S} \mathrm{II}_{1}\right] \lambda \lambda 6716,6731 / \mathrm{H} \alpha$ flux ratios vs. the emission-line widths (FWHM) for three to five independent positions in each of the 14 nebulae investigated in this paper. Note that the regions of the nebulae with the broadest lines have emission-line ratios most like shock-heated gas and least like normal giant $\mathrm{H}$ II regions (see Fig. 14). 
dropping back to 4-5 at larger radii. We infer from this that the dusty circumnuclear disk of M82 is slightly tipped, with the SE side being the near side.

\section{ii) Neutral Gas in the Nebula}

The extinction in the region of the superwind is $A_{V} \approx 0.8$ (see above). A normal gas-to-dust ratio would then imply an $\mathrm{H}$ I column density of about $1.3 \times 10^{21} \mathrm{~cm}^{-2}$. The $\mathrm{H} \mathrm{I}$ data of Crutcher, Rogstad, and Chu (1978) are consistent with this column density, suggesting that the cool material in the M82 nebula has a roughly normal gas-to-dust ratio. The total surface area of the region of the M82 nebula exhibiting double emission-line profiles is about $5 \mathrm{kpc}^{2}$. Together with the above $\mathrm{H}$ I column density, this would imply an $\mathrm{H}$ I mass of about $5 \times$ $10^{7} M_{\odot}$. This agrees well with Crutcher, Rogstad, and Chu (1978), whose H I $21 \mathrm{~cm}$ maps imply about $4 \times 10^{7} M_{\odot}$ of $\mathrm{H}_{\mathrm{I}}$ in this region. Note that similar amounts of $\mathrm{H}_{2}$ (Stark and Carlson 1984; Nakai et al. 1987) and hot X-ray-emitting gas (Watson, Stanger, and Griffiths 1984; Schaaf et al. 1989; Fabbiano 1988) are also present.

\section{iii) Luminosity}

We can use the above extinctions and our $\mathrm{H} \alpha$ images (MHV; Armus, Heckman, and Miley 1990) to calculate that the global extinction-corrected $\mathrm{H} \alpha$ luminosity of M82 is about $2 \times 10^{41} \mathrm{ergs} \mathrm{s}^{-1}$. This agrees with the earlier estimate of MHV and with the $\mathrm{H} \alpha$ luminosity implied by the $\mathrm{Br} \gamma$ measurements of Rieke et al. (1980). As we have discussed above, it is only the region located about 300-1200 pc above and below the circumnuclear disk in which both the kinematics and the emission-line ratios suggest that gas has been accelerated and shocked by a superwind. The approximate extinctioncorrected $\mathrm{H} \alpha$ luminosity of this region is only about $10 \%$ of the total, or about $2 \times 10^{40} \mathrm{ergs} \mathrm{s}^{-1}$. For typical gaseous nebulae, the total emission-line luminosity ( summed over all emission lines) is about 30 times the $\mathrm{H} \alpha$ luminosity. This, then, implies a total emission-line luminosity of $\approx 6 \times 10^{42} \mathrm{ergs} \mathrm{s}^{-1}$ from all of M82 and $\approx 6 \times 10^{41} \mathrm{ergs} \mathrm{s}^{-1}$ from the superwind region. For reference, the IR luminosity of M82 is $\approx 10^{44} \mathrm{ergs} \mathrm{s}^{-1}$.

\section{iv) Ionized Gas Mass}

The measured luminosities and electron densities, together with an assumption of case B conditions and $T \approx 10^{4} \mathrm{~K}$, allow us to estimate the mass of ionized gas in the M82 nebula. Taking an average density of $600 \mathrm{~cm}^{-3}$ for $r<300 \mathrm{pc}$ and 200 $\mathrm{cm}^{-3}$ for $300 \mathrm{pc}<r<1200 \mathrm{pc}$ yields masses of $7 \times 10^{5} M_{\odot}$ for the inner nebula and $2 \times 10^{5} M_{\odot}$ for the superwind region. These masses are much smaller than the masses of both cooler ( $\mathrm{H} \mathrm{I}$ and $\mathrm{H}_{2}$ ) and hotter (X-ray-emitting) gas that is apparently associated with the emission-line gas in the superwind region (see above). We will discuss the interrelationship between these various gas phases in $\S$ IV below.

\section{v) Filling Factors}

The filling factors implied by the masses and densities of the $\mathrm{H} \alpha$-emitting gas given above are about $10^{-4}-10^{-3}$ depending on the assumed three-dimensional structure (volume) of the nebula. Thus, the optical emission-line gas must consist of small clouds or thin sheets or filaments. This is quite consistent with the morphology of the nebula, which is exceedingly complex on scales of $10-100 \mathrm{pc}$.

\section{DISCUSSION}

\section{a) Introduction}

Our primary aims in this section are twofold. First, we will try to show (as quantitatively as possible) that the superwind model can naturally explain the diverse data described above, as well as important additional data in the literature. Second, we will use these data to determine or strongly constrain the basic properties of superwinds. The principal observational lines of evidence that support the superwind model are the kinematics of the ionized gas (particularly along the minor axes of the nearest edge-on FIRGs), the high observed gas pressures and the systematic dependence of the radial pressure profiles on the IR luminosity, the "shocklike" (LINER) emission-line spectra in the FIRG nebulae, and the existence of bright X-ray and optical emission-line nebulae located well out the plane of the stellar disks of FIRGs.

We will begin our discussion by showing that the conditions for the driving of a superwind are easily met in the starbursts that power our FIRGs $(\S \operatorname{IV} b)$. We will then $(\S \operatorname{IV} c)$ show how our data and related data in the literature can be used in a fairly straightforward manner to measure the basic superwind parameters. At the same time, we will demonstrate that these observationally derived parameters are in excellent quantitative agreement with the predictions of the model. We will next $(\S \mathrm{IV} d)$ present a simple kinematic model of the optical emission-line gas in the nearest edge-on FIRGs and show how it is consistent with the dynamics of a bipolar superwind. In $\S$ IV $e$, we will show that the high pressures observed in the optical nebulae, together with the modest X-ray luminosities of the FIRGs, provide strong evidence for ram pressure confinement of the emission-line clouds, and hence for the existence of a superwind. Next $\S \operatorname{IV} f$, we will show that superwinds are energetically adequate to power the observed optical and X-ray emission. We will also show that ( unlike the competing mechanisms of photoionization by normal O stars or AGN) shock heating by the superwind can also account in detail for the relative emission-line intensities and their radial dependences in the optical nebulae. Finally ( $\$ \mathrm{IV} g$ ), we will summarize the observational arguments that most of the observed superwinds are in the "blowout" phase, in which the wind has burst free of the ISM of the starburst galaxy. This will have a variety of important implications to be discussed in $\S \mathrm{V}$.

\section{b) Conditions for Driving a Superwind}

In this section we will show that the conditions to drive a superwind are easily met in well-studied FIRGs. These conditions are that the supernova rate be high enough to create a cavity of hot gas whose cooling time is much greater than its expansion time scale and that the wind is sufficiently powerful that it can ultimately "blow out" of the ISM of the starburst galaxy (i.e., not remain trapped as a hot bubble inside the galaxy).

In order to drive a superwind, the kinetic energy of supernovae and stellar winds must be efficiently thermalized-that is, 
the kinetic energy must be converted primarily into thermal energy of hot gas which permeates the starburst at base of the wind, with very little radiative loss. This condition will be met if the supernova rate is high enough to crease a dominant hot, rarified phase. We can evaluate this criterion using the arguments made by McKee and Ostriker (1977) concerning the structure of an ISM regulated by supernovae. McKee and Ostriker (1977) show that the hot phase of the ISM will have a filling factor:

$$
f_{\text {hot }}=1-\exp \left(-Q_{\mathrm{SNR}}\right),
$$

where

$$
Q_{\mathrm{SNR}}=3.3 E_{51}^{1.28} S_{-8} n_{0,100}^{-0.14} P_{7}^{-1.3} .
$$

Here $E_{51}$ is the energy per supernova in units of $10^{51} \mathrm{ergs}, S_{-8}$ is the supernova rate in units of $10^{-8}$ supernovae $\mathrm{pc}^{-3}$ year $^{-1}$, and $n_{0,100}$ and $P_{7}$ are the ambient density (in $100 \mathrm{~cm}^{-3}$ ) and pressure $\left(P / k\right.$ in units of $\left.10^{7} \mathrm{~K} \mathrm{~cm}^{-3}\right)$ in the nucleus. The supernova rate in the best-studied FIRGs M82 and NGC 253 is estimated to be one supernova per 3-10 yr (Rieke et al. 1980; Kronberg, Biermann, and Schwab 1985; Antonucci and Ulvestad 1988; $\S$ IV $c$ below). These occur in a volume of about $10^{7} \mathrm{pc}^{3}$, and hence $S_{-8} \approx 1-3$. The present mean gas densities in the M82 and NGC 253 starbursts are several hundred per $\mathrm{cm}^{3}$ (largely in molecular gas). The present value of $P_{7}$ is of order unity, and its value must have been much smaller just prior to the onset of the starburst. We therefore conclude that the starburst will lead to an ISM that is dominated (in terms of filling factor, but not mass) by the hot supernova-heated phase.

Within the hot cavity so created, it is easy to show that radiative losses are negligible. Following CC, the temperature of the hot phase is about $7 \times 10^{7} \mathrm{~K}$. Our measurements of a typical central pressure of $3 \times 10^{-9}$ dynes $\mathrm{cm}^{-2}$ then imply a central density of $0.13 \mathrm{~cm}^{-3}$ for the hot phase. Both this temperature and density are in excellent agreement with estimates based on the X-ray data (e.g., Schaaf et al. 1989). These parameters lead to a radiative cooling time of about $4 \times 10^{8} \mathrm{yr}$, much longer than the sound-crossing time in the central region ( few $\times 10^{5}$ yr). More generally, Mac Low and McCray (1988, hereafter MM) have shown that the ratio of cooling time to dynamical/ sound-crossing time in a supernova-driven wind imbedded in an exponential atmosphere with a scale height $H_{\mathrm{kpc}}($ in $\mathrm{kpc}$ ) is given by

$$
t_{\mathrm{cool}} / t_{\mathrm{dyn}}=290 n_{0}^{-1.1} d E / d t_{43}^{0.61} H_{\mathrm{kpc}}^{-1.7} \xi^{-1.6},
$$

where $n_{0}$ is the midplane density $\left(\mathrm{cm}^{-3}\right), d E / d t_{43}$ is the rate at which the starburst injects kinetic energy (in $10^{43} \mathrm{ergs} \mathrm{s}^{-1}$ ), and $\xi$ is the metal abundance relative to solar. For the parameters appropriate to the M82 or NGC 253 starbursting molecular disks $\left(n_{0} \approx\right.$ several hundred, $d E / d t_{43} \approx$ unity, $H_{\mathbf{k p c}}<0.1$ ), it is clear that $t_{\mathrm{cool}} \gg t_{\mathrm{dyn}}$, and the radiative losses are therefore negligible.

MM and MMN have further shown that a wind will blow out of an exponential atmosphere if the dimensionless wind luminosity $\Lambda>100$, where

$$
\Lambda=30 d E / d t_{43} H_{\mathrm{kpc}}^{-2} P_{7}^{-3 / 2} n_{0}^{1 / 2} .
$$

Taking the above values for these parameters, it therefore appears that the superwinds will blow out of starburst galaxies. We will summarize the observational evidence that at least some of our FIRGs are in the blow-out phase in $\S \mathrm{IV} g$ below.

\section{c) Measuring the Basic Starburst and Superwind Parameters}

In this section, we will show how our data and data from the literature can be used to measure the basic parameters characterizing the starbursts and the superwinds. The strikingly good agreement between the predictions of the superwind theory and these observationally derived quantities represents perhaps the strongest evidence in favor of superwinds.

\section{i) Star-Formation Rate}

In order to estimate the rate at which the starburst is injecting mass, energy, momentum, and metals into the superwind, it is first necessary to know the rate at which massive stars are forming in the starburst galaxy. The data that we will use to estimate the star-formation rate (SFR) are the bolometric luminosity of the starburst (taken to be $\approx$ the total IR luminosity) and the Lyman continuum luminosity (taken from IR or radio $H_{\text {I }}$ recombination lines or free-free radio continuum measurements). To translate these quantities accurately into an SFR requires knowledge of the initial mass function. We will assume a normal Salpeter IMF extending from $M=0.1-$ $100 M_{\odot}$, but note that the estimated SFRs would be about 3 times smaller if a lower mass cutoff of $3.5 M_{\odot}$ is applicable (as advocated by Rieke et al. 1980). For simplicity, we will assume that the SFR has been constant over the lifetimes of the highmass stars $\left(\approx 10^{7} \mathrm{yr}\right.$ for $\left.M=10 M_{\odot}\right)$.

These assumptions allow us to adopt the relation between IR luminosity and star-formation rate derived by Hunter $e t$ al. (1986):

$$
\mathrm{SFR}=26 L_{\mathrm{IR}, 11} M_{\odot} \mathrm{yr}^{-1},
$$

where $L_{\mathrm{IR}, 11}$ is the total IR luminosity (in units of $10^{11} L_{\odot}$ ) derived (in order of preference) from two-temperature fits to the IRAS data by Rice et al. (1988) or from one-temperature fits following Soifer et al. (1987) - see the Notes to Table 1. This is equivalent to taking $L_{\mathrm{TR}} \approx L_{\mathrm{BOL}}$ (e.g., setting the $\beta$ parameter of Hunter et al. $=1)$. Equation 5, for example, yields a SFR of about 8 or $9 M_{\odot} \mathrm{yr}^{-1}$ for M82 and NGC 253.

We can also estimate the current SFR using estimates of the Lyman continuum luminosity. The relationships in Kennicutte (1983) can be rewritten as

$$
\begin{aligned}
& \operatorname{SFR}\left(>10 M_{\odot}\right)=2 Q_{\mathrm{Ly}, 54} M_{\odot} \mathrm{yr}^{-1}, \\
& \operatorname{SFR}(\text { total })=12 Q_{\mathrm{Ly}, 54} M_{\odot} \mathrm{yr}^{-1},
\end{aligned}
$$

where $Q_{\mathrm{Ly}, 54}$ is the Lyman continuum luminosity (in units of $10^{54}$ photons s ${ }^{-1}$ ) and where we have used Kennicutt's "extended Miller-Scalo" (close to Salpeter) IMF over the mass range $0.1-100 M_{\odot}$. In $\mathrm{M} 82$, the $\operatorname{Br} \alpha$ luminosity implies that $Q_{\mathrm{Ly}} \approx 3 \times 10^{53} \mathrm{~s}^{-1}$. Thus, the total SFR in M82 would be $\approx 4$ $M_{\odot} \mathrm{yr}^{-1}$. We list the SFRs estimated from the IR luminosity 
and (when available) from the Lyman continuum luminosity in Table 5. In Arp 220, the SFR derived from the IR is more than one order of magnitude larger than that derived from the $\operatorname{Br} \alpha$ luminosity. For the other FIRGs, the SFRs derived from the two data types are consistent to within a factor of a few.
More generally, DePoy (1987) and Ho, Beck, and Turner (1990) have both shown that the $\mathrm{Br} \alpha$ luminosity is reasonably well-correlated with IR luminosity in FIRGs, and that the ratio of $\mathrm{Br} \alpha$ to IR luminosity is similar to the ratio in Galactic $\mathrm{H}$ II regions.

TABLE 5

STARBURST AND SUPERWIND PARAMETERS

\begin{tabular}{|c|c|c|c|c|c|c|c|c|c|c|c|c|c|}
\hline $\begin{array}{l}\text { Galaxy } \\
\text { (1) }\end{array}$ & $\begin{array}{c}\mathrm{SFR}_{\text {IR }} \\
(2)\end{array}$ & $\begin{array}{c}\mathrm{SFR}_{\mathrm{LYC}} \\
\text { (3) }\end{array}$ & $\begin{array}{c}d E / d t_{\text {kin, } 42} \\
\text { (4) }\end{array}$ & $\begin{array}{c}d E / d t_{*, 42} \\
(5)\end{array}$ & $\begin{array}{c}P \Delta V / \Delta t_{42} \\
(6)\end{array}$ & $\begin{array}{c}P \Delta V_{56} \\
(7)\end{array}$ & $\begin{array}{c}d p / d t_{\mathrm{obs}, 35} \\
(8)\end{array}$ & $\begin{array}{c}d p / d t_{*, 33} \\
\text { (9) }\end{array}$ & $\begin{array}{c}d M / d t * \\
(10)\end{array}$ & $\begin{array}{l}\tau_{\mathrm{SNe}} \\
(11)\end{array}$ & $\begin{array}{l}P_{0,-9} \\
(12)\end{array}$ & $\begin{array}{c}r_{*, \mathrm{P}} \\
(13)\end{array}$ & $\begin{array}{l}r_{*} \text { obs } \\
(14)\end{array}$ \\
\hline IRAS 00812-7112 $\ldots \ldots$ & $\approx 2000$ & & & $\approx 600$ & & & & $\approx 50$ & $\approx 300$ & 0.06 & & & \\
\hline NGC 1222 . & 8 & 15 & . & 2 & 3 & 2 & 0.5 & 0.2 & 1.2 & 17 & 2 & 450 & 700 \\
\hline NGC 1614 & 70 & 120 & .. & 2 & 5 & 7 & 1.1 & 1.5 & 10 & 2 & 5 & 460 & 500 \\
\hline M82 ...... & 9 & 4 & 2 & 2 & 1.4 & $1.2 ; 10$ & $0.4 ; 0.4$ & 0.2 & 1.3 & 15 & 4 & 270 & 300 \\
\hline NGC $3079 \ldots$ & 8 & 2 & 9 & 2 & 1 & 1 & $0.8 ; 2$ & 0.2 & 1.2 & 16 & 2 & 590 & 600 \\
\hline NGC $4194 \ldots \ldots \ldots \ldots \ldots$ & 19 & 15 & $\ldots$ & 5 & 4 & 4 & 0.8 & 0.5 & 3 & 7 & 3 & 470 & 200 \\
\hline NGC $4945 \ldots \ldots \ldots \ldots \ldots$ & 11 & $\ldots$ & 3 & 3 & 0.8 & 0.3 & $0.13 ; 0.5$ & 0.2 & 1.6 & 12 & 1.6 & 210 & $\approx 100$ \\
\hline Mrk $266 \ldots \ldots \ldots \ldots \ldots$ & 60 & 30 & $\ldots$ & 16 & 60 & 250 & 12 & 1.4 & 9 & 2 & 3 & 2000 & \\
\hline Mrk 273 & 330 & 70 & $\ldots$ & 90 & 40 & 130 & 8 & 8 & 50 & 0.4 & 1.6 & 2100 & 2000 \\
\hline $\operatorname{Arp} 220 \ldots \ldots \ldots$ & 340 & 30 & $\ldots$ & 90 & $\approx 60$ & 1000 & $\ldots$ & 8 & 50 & 0.4 & 100 & & $<500$ \\
\hline NGC $6240 \ldots \ldots \ldots \ldots$ & 140 & $30 ; 130$ & $\ldots$ & 40 & 17 & 60 & 7 & 3 & 30 & 1.0 & 2 & 1700 & 1800 \\
\hline
\end{tabular}

NoTES.-Key to cols. (2)-(14) follows:

Col. (2).-Star-formation rate in $M_{\odot} \mathrm{yr}^{-1}$ derived from the IR luminosity using eq. (5) (see text).

Col. (3). - Star-formation rate in $M_{\odot} \mathrm{yr}^{-1}$ derived from the Lyman continuum luminosity using eq. (6b) (see text). When possible (NGC 253, NGC 1614, M82, NGC 4194, and Arp 220), the Lyman luminosities were derived from the extinction-corrected Br $\alpha$ luminosities published/compiled in DePoy 1987 and Ho, Beck, and Turner 1990. For NGC 1222, NGC 3079, Mrk 266, and Mrk 273, the Lyman luminosities were estimated from the global H $\alpha$ [N II] fluxes in Armus, Heckman, and Miley 1990 , corrected for dust extinction and [N II] contamination using the $\mathrm{H} \alpha / \mathrm{H} \beta$ and [N $\mathrm{r}] / \mathrm{H} \alpha$ ratios in Armus, Heckman, and Miley 1989. For NGC 6240 we give a value for the SFR based on the nuclear $\mathrm{Br} \alpha$ line, followed by the rate derived from the extinction-corrected global $\mathrm{H} \alpha$ luminosity (because the nebula is much larger than the aperture size used to measure the $\mathrm{Br} \alpha$ ). For NGC 3256, we used the $\mathrm{Br} \gamma$ luminosity from Kawara, Nishida, and Gregory 1987, corrected for an extinction of $0.8 \mathrm{mag}$ at $\mathrm{Br} \gamma$ (the mean value in DePoy's (1987) sample of IR galaxies).

Col. (4).-Estimate of the wind kinetic energy flux (in units of $10^{42} \mathrm{ergs} \mathrm{s}^{-1}$ ), based on the "wind-driven bubble" model for the kinematics of the gas along the minor axes of NGC 253, M82, NGC 3079, and NGC 4945 (e.g., using eq. [8] in the text). For each galaxy, we have used $n_{0} \approx 2 \mathrm{~cm}^{-3}$ for the ambient preshock density, based on the postshock densities measured with the [S II] doublet (see Shull and McKee 1979). We have set $v$ equal to half the average velocity separation of the two velocity components in the region of double-peaked emission-line profiles. We have set $r=$ the projected separation between the nucleus and the outermost boundary of the region of double-peaked profiles.

Col. (5).-Predicted kinetic energy output of the starburst derived from eq. (11) (in units of $10^{42} \mathrm{ergs} \mathrm{s}^{-1}$ ), using the measured IR luminosity.

Col. (6). - Estimate of the kinetic energy flux in the wind (in units of $10^{42} \mathrm{ergs}^{-1}$ ). We have used the radial pressure profiles measured with the [S n] doublet to calculate $P \Delta V$ in the nebulae out to the radius where $n_{e} \approx 100 \mathrm{~cm}^{-3}$ (see col. [7]). We have then divided this energy by the estimated dynamical time for this region (taking an outflow velocity given by the biconic outflow models in Table 5 for NGC $253, \mathrm{M} 82$, and NGC 4945, and $\approx 400 \mathrm{~km} \mathrm{~s}^{-1}$ for the other galaxies). For Arp 220, we used $P \Delta V$ from the X-ray data and took the dynamical time to be the expansion time for the western H $\alpha$ bubble.

Col. (7).-Estimates of the energy content of the nebulae (in units of $10^{56} \mathrm{ergs}$ ), derived from the volume integral of the radial pressure profiles out to the radius where $n_{e} \approx 100 \mathrm{~cm}^{-3}$. For M82, NGC 253, and Arp 220, we also give values for $P \Delta V$ estimated for their large X-ray nebulae. We have taken a volume filling factor of unity and $T \approx 10^{8} \mathrm{~K}$ for this hot gas. The X-ray data yield larger energies than the [S II] data in M82 and NGC 253 because the volume over which the integral is performed is much larger in the case of the X-rays (see Fabbiano 1988 and Schaaf et al. 1989 for the details).

Col. (8). - Momentum flux in the wind (in units of $10^{35}$ dynes) derived from the radial pressure profiles using eq. (10) in the text evaluated at $r_{n 100}$. For NGC 253, M82, NGC 3079, and NGC 4945, we also give the value for $d p / d t$ derived from the kinematics of the gas, using the "wind-blown bubble" model (eq. [9] in the text). See the Notes for col. (4) for details.

Col. (9).- The predicted momentum flux (in units of $10^{35}$ dynes) from the starburst as estimated by eq. (11) in the text using the measured IR luminosity.

Col. (10).-Predicted mass flux $\left(M_{\odot} \mathrm{yr}^{-1}\right)$ of metal-rich superwind fluid generated by the starburst (no entrainment assumed). This is based on eq. (11) in the text and the IR luminosity.

Col. (11).-Average time between supernovae (yr), predicted from the IR luminosity using eq. (11) in the text.

Col. (12).-Central pressure (in units of $10^{-9}$ dynes $\mathrm{cm}^{-2}$ ) implied by the measured central electron densities $\left(n_{e}\right)$ shown in Fig. 13. The conversion from density to pressure takes $n_{\mathrm{TOT}} \approx 3 n_{e}$ and $T \approx 10^{4} \mathrm{~K}$.

Col. (13). - Starburst radius (pc) derived from the radial pressure profiles as measured with the [S II] doublet. We have used eq. (12) for this purpose, taking the values in col. (8) for $d p / d t$ and the central pressures from col. (12).

Col. (14).-Approximate radii of the starbursts $(\mathrm{pc})$, as measured with mid- and far-IR continuum emission, nonthermal radio emission, optical and IR recombination and $\mathrm{H}_{2}$ lines, and millimeter-wave (CO) lines. The references used to derive these radii are: Antonucci and Ulvestad 1988 and Canzian, Mundy, and Scoville 1988 for NGC 253; Armus, Heckman, and Miley 1990 for NGC 1222 and NGC 1614; Nakai et al. 1987, Lo et al. 1987, and Lester et al. 1990 for M82; Young, Claussen, and Scoville 1987 for NGC 3079; Graham et al. 1987 for NGC 3256; Gehrz, Sramek, and Weedman 1983, Joy et al. 1989, and Nakagawa et al. 1989 for NGC 3690 (a confusing "triple" source at IR and radio wavelengths, for which we therefore give only an approximate size of a kpc); Armus, Heckman, and Miley 1990 for NGC 4194; Brock et al. 1988 and Moorwood and Glass 1984 for NGC 4945 (and see text in $\S$ III); Armus, Heckman, and Miley 1990 for Mrk 273; Becklin and Wynn-Williams 1987 for Arp 220; Armus, Heckman, and Miley 1990 and Lester, Harvey, and Carr 1988 for NGC 6240 . 


\section{ii) Time Scales and Lifetimes}

We can use our observations of the kinematics of the ionized gas in typical FIRGs to estimate the dynamical time scales for the nebulae. These times are given by

$$
t_{\mathrm{dyn}, 6} \approx 10 r_{\mathrm{neb}, \mathrm{kpc}} / \delta v_{\mathrm{neb}, 100} \mathrm{Myr}
$$

where $\delta v_{\text {neb,100 }}$ is the estimated expansion velocity of the nebula (in units of $100 \mathrm{~km} \mathrm{~s}^{-1}$. Taking this to be half the velocity separation of the double line profiles in M82, NGC 253, NGC 3079, NGC 4945, and Arp 220, and the average HWHM of the lines in the NGC 6240 and IRAS 00181-7112 nebulae yields dynamical times of about 3-10 $\times 10^{6} \mathrm{yr}$ for the first four galaxies and time scales of $30-100 \times 10^{6} \mathrm{yr}$ for the latter three.

These times can be compared to observationally derived estimates of the duration of the starbursts. The mass of molecular and atomic gas in the star-bursting region of M82 is about $10^{8} M_{\odot}$, compared to a total dynamical mass of abut $6 \times 10^{8}$ $M_{\odot}$ (Lo et al. 1987). For a star-formation rate of $9 M_{\odot} \mathrm{yr}^{-1}$, the gas depletion time is about $11 \times 10^{6} \mathrm{yr}$. The corresponding time scale for Arp 220 is $\approx 100 \times 10^{6}$ yr for a total SFR of $\approx 100 M_{\odot} \mathrm{yr}^{-1}$. Finally, the analysis of Rieke et al. (1980, 1985 ) yields characteristic starburst ages of several tens of Myr in the cases of M82, NGC 253, Arp 220, and NGC 6240. We therefore conclude that the dynamical ages of the nebulae are comparable to the ages of the starbursts (as expected in the superwind model).

\section{iii) Injection/Outflow Rates}

Our spectroscopic data can be used in several independent ways to measure or strongly constrain the rates at which superwinds carry mass, momentum, and energy out of the FIRGs. These can be compared to similar rates estimated from the $\mathrm{X}$-ray data and to the predicted injection rates of the starbursts.

The first technique for estimating outflow rates is to analyze the kinematics of the gas along the minor axes of the edge-on FIRG's in the context of dynamical models of wind-blown bubbles ( see CMW). This treatment is strictly accurate only for a wind expanding into a uniform, spherically symmetric medium during the phase before the wind "blows out." We will therefore use this technique only in an order-of-magnitude sense. The radius (in $\mathrm{kpc}$ ) and velocity (in $10^{2} \mathrm{~km} \mathrm{~s}^{-1}$ ) of an energy-conserving bubble inflated by energy injected at a constant rate and expanding into a uniform medium with an ambient density $n_{0}\left(\mathrm{~cm}^{-3}\right)$ are related to the energy injection rate by

$$
d E / d t \approx 3 \times 10^{41} r_{\mathrm{kpc}}^{2} v_{100}^{3} n_{0} \operatorname{ergs~s}^{-1}
$$

If radiative losses are important and the bubble is momentumconserving, the analogous expression for the momentum injection rate is

$$
d p / d t \approx 2 \times 10^{34} r_{\mathrm{kpc}}^{2} v_{100}^{2} n_{0} \text { dynes }
$$

We have used these equations to estimate the momentum and energy injection rates in the four nearby edge-on FIRGs (M82, NGC 253, NGC 3079, and NGC 4945). Note that $n_{0}$ is the density in the ambient (undisturbed) medium just outside the expanding wall of the wind-blown bubble. We can estimate this by using shock models (e.g., Shull and McKee 1979) to relate the $n_{e}$ measured in the postshock $S$ II zone to the preshock density. This procedure yields values $n_{0} \approx$ several per $\mathrm{cm}^{-3}$. The implied values for $d E / d t$ and $d p / d t$ are on the order of a few $\times 10^{42}$ ergs $\mathrm{s}^{-1}$ and several $\times 10^{34}$ dynes, respectively, for these four relatively low power FIRGs (see Table 5).

A second technique for determining $d p / d t$ from our data is to use the radial pressure profiles displayed in Figure 13. If we assume that (outside the central starburst region) these profiles are regulated by the ram pressure in the superwind flowing out into a solid angle $\Omega$, then momentum flux in the wind is given by

$$
d p / d t \approx 1.2 \times 10^{35} r_{\mathbf{k p c}}^{2} P_{-9}(r)(\Omega / 4 \pi) \text { dynes },
$$

where $P_{-9}(r)$ is the pressure (in units of $10^{-9}$ dynes $\mathrm{cm}^{-2}$ ) at a radius $r_{\mathrm{kpc}}$. We have used this equation to calculate $d p / d t$ for the 12 FIRGs shown in Figure 13 . We adopt $\Omega \approx 4 \pi$, since ( 1 ) the $P(r)$ profiles were averaged over arbitrary position angles for most of our FIRGs, and (2) the pressure profiles perpendicular to the wind outflow axis for the four edge-on FIRGs (M82, NGC 253, NGC 3079, and NGC 4945) imply that $\Omega / 4 \pi>0.5$ (see Table 4 ). The values we then derive for $d p / d t$ range from few $\times 10^{34}$ dynes for low-power FIRGs like M82 (in good agreement with the values calculated from Eq. [9]) to $\approx 10^{36}$ dynes for powerful FIRGs like Mrk 266 and Mrk 273. The various values are listed and compared in Table 5.

The measured radial pressure profiles for FIRGs can be used to provide an independent estimate of the energies and energy flow rates associated with the superwind. Integrating the radial pressure profiles displayed in Figure 13 over the volume of each nebula yields $P \Delta V$, which represents the kinetic (thermal) energy of the wind fluid if the optically emitting clouds are confined by the ram (static thermal) pressure of this fluid. They also represent the adiabatic work done by the wind. As listed in Table 5, these energies range from about $10^{56}$ ergs for the low-power FIRGs like M82, and NGC 253 to about $10^{58}$ ergs for high-power FIRGs like Mrk 266 and Mrk 273. Dividing these energies by the dynamical time scales for the nebulae then gives rough time-averaged values for $d E / d t$ (also given in Table 5). These are of order $10^{42} \mathrm{ergs} \mathrm{s}^{-1}$ for low-power FIRGs like M82, and range up to several $\times 10^{43} \mathrm{ergs} \mathrm{s}^{-1}$ in the powerful FIRGs.

A similar estimate of total energy and energy flux can be made for the three FIRGs in our sample with published X-ray maps (M82, NGC 253, and Arp 220). The total thermal energies in the $\mathrm{X}$-ray plasma are $\approx 10^{57}$ ergs for the first two galaxies, and $\approx 10^{59}$ ergs in Arp 220 , assuming it has a filling factor of unity and a temperature of $10^{8} \mathrm{~K}$ (see Table 5 for details).

The values for $d p / d t$ and $d E / d t$ we have determined can be used to estimate the mass outflow rates in the superwind fluid, provided that the velocity of the superwind fluid is known. We emphasize that in the superwind model, this velocity is different from, and much higher than, the velocities observed in the swept-up ambient gas visible optically (see Fig. 1 and $\S$ IV $a$ above). As we will describe below, the superwind model predicts a wind terminal velocity of about $2000-3000 \mathrm{~km} \mathrm{~s}^{-1}$. The 
only observationally derived estimate comes from Seaquist, Bell, and Bignell's (1985) observations and model of the nonthermal radio emission associated with the wind of M82. They interpreted the systematic trend for the radio spectrum to steepen with increasing distance from the starburst disk as due to synchrotron cooling of relativistic plasma being convected outward by a wind. They then calculated a wind velocity of $\approx 2000 \mathrm{~km} \mathrm{~s}^{-1}$ from the synchrotron loss times. Taking a wind speed of $2000 \mathrm{~km} \mathrm{~s}^{-1}$ and the estimated values for $d p / d t$ and $d E / d t$ given above and in Table 5 implies that $d M / d t \approx$ a few $M_{\odot} \mathrm{yr}^{-1}$ in low-power FIRGs like M82, and ranges up to $\approx 50$ $M_{\odot} \mathrm{yr}^{-1}$ in powerful FIRGs like Arp 220 and Mrk 273. We emphasize that these rates refer to the superwind fluid itself (generated in the starburst from supernova ejecta and stellar winds) and do not include any ambient material that is swept up by the wind.

The amount of ambient material likely to be entrained by the wind can only be estimated in an approximate sense. We will argue in $\S$ IV $d$ below on kinematic grounds that the optical nebulae associated with superwinds may consist of material entrained into the outer boundary of an outflowing superwind having a biconic or cylindrical morphology. In an outflow of this kind, the wind is in effect a poorly collimated jet. The entrainment process has been studied quantitatively by De Young $(1981,1986)$ in the context of radio-synchrotron jets. His arguments lead to estimated entrainment rates of a few $M_{\odot}$ $\mathrm{yr}^{-1}$ for the low power FIRGs up to about $100 M_{\odot} \mathrm{yr}^{-1}$ for Arp 220. However, these numbers are uncertain by about one order of magnitude.

Having estimated outflow rates using a variety of observational data, we can now compare these rates to the injection rates predicted from supernovae and stellar winds in the starburst (assuming the IMF and the SFRs calculated in $\S \mathrm{IV} c$ [iii] above). Elson, Fall, and Freeman (1989, hereafter EFF) have estimated the supernova rate and the mass/metal/energy injection rates as functions of the slope and upper mass cutoff of the IMF. Using a Salpeter IMF with an upper mass cutoff of $100 M_{\odot}$, a star-formation rate of $10 M_{\odot} \mathrm{yr}^{-1}$ (approximately as in M82, for example) would produce one supernova every 15 yr and time-averaged mass, momentum, and energy injection rates from both stellar winds and supernovae of about $1.5 M_{\odot}$ $\mathrm{yr}^{-1}, 2.3 \times 10^{34}$ dynes and $2.7 \times 10^{42}$ ergs s$^{-1}$, respectively. These parameters correspond to a mean injection velocity and wind terminal velocity of about $2400 \mathrm{~km} \mathrm{~s}^{-1}$. If we adopt the relation in equation (5) to relate the SFR to the IR luminosity, then the EFF models imply

$$
\begin{aligned}
d M / d t & =4 L_{\mathrm{IR}, 11} M_{\odot} \mathrm{yr}^{-1}, \\
d p / d t & =6 \times 10^{34} L_{\mathrm{IR}, 11} \text { dynes }, \\
d E / d t & =7 \times 10^{42} L_{\mathrm{IR}, 11} \mathrm{ergs} \mathrm{s}^{-1}, \\
\nu(\mathrm{SNE}) & =0.2 L_{\mathrm{IR}, 11} \mathrm{yr}^{-1} .
\end{aligned}
$$

We summarize all the various estimates of these outflow and injection rates for our FIRGs in Table 5. Not only is there good overall agreement between values derived in several independent ways from the data, but these values for the outflow also agree well with the injection rates implied by the estimated star-formation rates. The excellent quantitative agreement between the predictions of the superwind model, and the data are illustrated graphically in Figure 17, where we plot the radius at which the measured electron density reaches $100 \mathrm{~cm}^{-3}\left(r_{n 100}\right)$ versus the IR luminosity $\left(L_{\mathrm{IR}}\right)$ for the 12 FIRGs with measured radial pressure profiles. At this radius, the pressures are dominated by ram pressure. Thus, the model predicts that $r_{n 100}$ should scale as $L_{\mathrm{IR}}^{1 / 2}$ and be about $1.3 \mathrm{kpc}$ for $L_{\mathrm{IR}}=10^{11} L_{\odot}$. The predicted relationship is shown in Figure 17 and provides a good fit to the data.

\section{iv) The Sizes of the Starbursts}

The central pressure inside the starburst (the region of mass and energy injection, and hence the region inside the sonic radius of the wind) is just the static pressure of the hot fluid that has been produced by shock heating in high-speed collisions between material ejected by supernovae and stellar winds. As discussed by $\mathrm{CC}$, this pressure is determined by the size of the starburst and the rate at which it injects momentum. Thus, our estimates of $d p / d t$ from $\S \operatorname{IV} c$ (iii) above and the central gas pressures given in Figure 13 allow us to calculate the size of the starburst. Following $\mathrm{CC}$, it is easy to show that the radius of the starburst is

$$
r_{*}=0.93 d p / d t_{35}^{1 / 2} P_{0,-9}^{-1 / 2} \mathrm{kpc},
$$

where the momentum injection rate is in units of $10^{35}$ dynes and the central pressure is in units of $10^{-9}$ dynes $\mathrm{cm}^{-2}$.

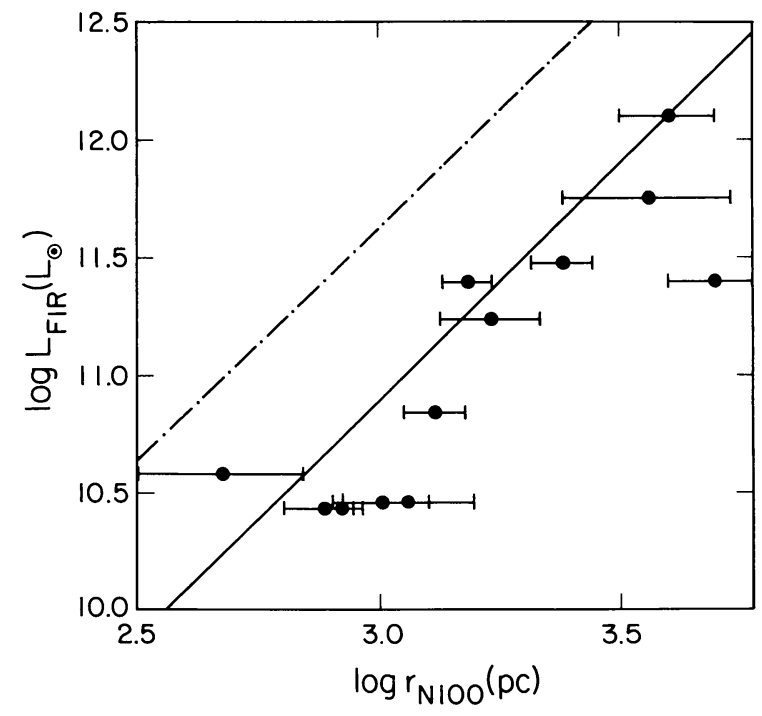

FIG. 17.-A log-log plot of the IR luminosity of the galaxy ( see Table 1) vs. $r_{\mathrm{N} 100}$, the radius at which $n_{e} \approx 100 \mathrm{~cm}^{-3}$ in the optical emission-line gas. The dots with associated error bars represent the 12 galaxies for which density profiles could be measured (see also Fig. 13). The solid diagonal line represents the relationship predicted by the superwind model, based on the following assumptions: the density-to-pressure conversion for the emission-line gas is as given by $P=3 \times 10^{4} n_{e} k$, the emission-line clouds are in pressure-balance with the ram pressure of the superwind, the scaling between the IR luminosity of the galaxy and the wind momentum flux is given by eq. (11b), and the wind flows into $4 \pi \mathrm{sr}$ ( $\operatorname{see} \S \mathrm{IV} c$ [iii]). The dashed diagonal line represents the relationship expected instead if the pressure measured in the emission-line gas were equal to the radiation pressure produced by the starburst ( $\operatorname{see} \S$ IV $d[i]$ ). 
The sizes we derive are given in Table 5 and range from radii of a few hundred pc for objects like M82 to $\approx 2 \mathrm{kpc}$ for some of the most powerful FIRGs. Such size scales agree remarkably well with the directly measured sizes of the starbursts (see Table 5 and Fig. 18).

\section{d) A Kinematic Model for the Outflows}

The properties of the kinematically disturbed gas are remarkably similar along the minor axes of M82, NGC 253, and NGC 4945. Specifically:

a) The gas is brighter on the near side of the slightly tipped galaxy disk, as expected, if this disk is dusty. This effect is also present in NGC 3079. Note that in M82 we have used the Balmer decrement to show directly that the fainter gas on the far side does suffer much heavier extinction.

b) The region of split lines extends out to a radial distance of about $1 \mathrm{kpc}$ along the galaxy minor axis and has a width (parallel to the galaxy major axis) of $\approx 300-1000 \mathrm{pc}$. The "bubbles" in NGC 3079 and Arp 220 and the "hourglass" in NGC 6240 have similar aspect ratios, but are about one order of magnitude larger in the latter two galaxies (see HAM).

c) The region of split lines corresponds to a region of strongly enhanced optical line emission, even though the line emission extends well beyond the region of split lines (Table 4). This is also true in NGC 3079.

d) The faint gas outside the region of split lines is kinematically quiescent (also true in NGC 3079).

e) The region of split lines has an edge-brightened morphology (the surface brightness of the gas is highest along the sides of the region). The Arp 220 and NGC 3079 "bubbles" and the NGC 6240 "hourglass" are two additional examples of this phenomenon.

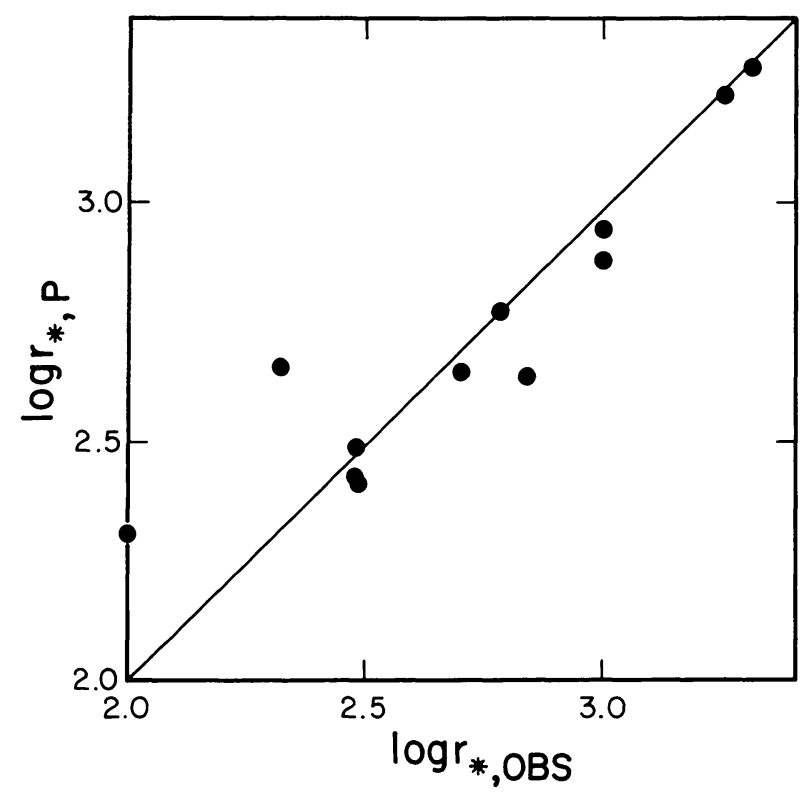

FIG. 18.-A log-log plot of $r_{*, \text { obs }}$ (the observed radius of the starburst) vs. $r_{*, P}$ (the radius of the starburst predicted by the shape and amplitude of the measured radial pressure profiles). Both radii are given in pc. They are listed in Table 5 and described further in the notes to Table 5 and in $\S$ IV $c$ [iv]. The solid line represents $r_{*, \text { obs }}=r_{*, P}$. f) The split lines on the near side of the galaxy disk consist of a component near the systemic velocity and a blueshifted component.

g) In M82, the split lines on the far side of the galaxy disk consist of a component near the systemic velocity and a redshifted component. No region of split lines is detected on the far side in NGC 253 and NGC 4945 (presumably due to heavy extinction).

$h$ ) The component near the systemic velocity is significantly brighter (by typically a factor of 2-5) than the blue- or redshifted component.

Properties $(a)-(g)$ above can be understood in terms of a model (Fig. 19) in which the optically emitting gas is flowing radially outward along the walls of twin cones, each of whose apex is at the galaxy's nucleus and whose axis of symmetry lies along the minor axis of the galaxy. Because the galaxies are not seen exactly edge-on, this axis of symmetry is tipped with respect to the plane of the sky. The front surface of the near side cone produces the blueshifted component, while the component near $v_{\text {sys }}$ comes from the back. Similarly, the front surface of the far side cone produces a component near $v_{\text {sys }}$, and the back surface produces a redshifted component. Note that a spherically expanding bubble will produce red-and blueshifted components with velocities symmetric about $v_{\text {sys }}$ on both the near and far sides of the tipped disk (which does not agree with properties $(f)$ and $(g)$ above, but does agree with the kinematics of the NGC 3079 bubbles).

Adopting a biconic geometry, we can determined the angle between the cone and sky axes from measurements of both the cone opening angle and the ratio of the velocities (with respect to systemic) on the front and back of the cone. Knowing the cone opening angle and its orientation, we can deproject the measured velocities to determine the intrinsic outflow speeds of the optically emitting gas along the cone wall. The details concerning this procedure and the results it yields are given in Table 6 for M82, NGC 253, and NGC 4945. We find that the cone axes are tipped about $25^{\circ} \pm 10^{\circ}$ relative to the sky plane and the intrinsic outflow speeds are about $300-500 \mathrm{~km} \mathrm{~s}^{-1}$.

A hollow-cone type outflow pattern is physically plausible in the superwind. As noted earlier, when the ambient medium is not spherically symmetric (i.e., has a disklike form) the wind will inflate cylindrical or conelike cavities along the disk's minor axis (see TI; Schiano 1985; MMN 1989; Norman and Ikeuchi 1989). The velocity vectors of the shocked ambient gas are not precisely radial (as in the idealized case in Fig. 19), but do have a quasi-radial orientation with components both parallel to and perpendicular to the symmetry axis of the outflow ( see Fig. 1).

Property $(\mathrm{h})$ above is not readily explicable in terms of the simple biconic outflow picture. It cannot be due to extinction from dust inside the cone because then the blueshifted kinematic component on the near side cone (which arises on the cone's front surface) should be brighter than the component near the systemic velocity (which arises on the back surface). If emission lines arise in clouds that themselves are dusty and the optical line emission arises on the side of the cloud facing into the wind (as expected if the clouds are shock heated by the wind or photoionized by the starburst), then for the near side cone the kinematic component near $v_{\text {sys }}$ should be brighter than the blueshifted component. This agrees with the observa- 


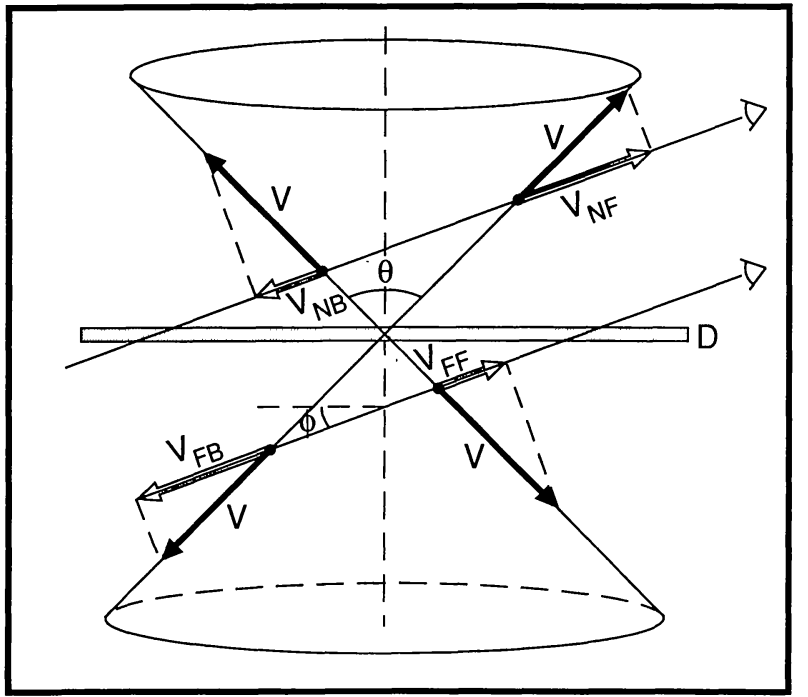

FIG. 19.-Schematic of outflow along a biconic surface centered on the nucleus of a disk-shaped galaxy, with the cone's symmetry axis (dashed vertical line) along the minor axis of the disk. The disk is indicated by the rectangular region labeled " $\mathrm{D}$," and the structure is viewed by an observer (stylized eyeball) whose line of sight makes an angle $\phi$ with the plane of the galaxy disk. The opening angle of each cone is $\theta$, and the gas flows along the surface of the cone at a uniform velocity $v$ (solid arrows). The line of sight through the cone on the near side of the galaxy disk yields a double-peaked emission line with the gas on the front surface, producing a component that is strongly blueshifted (open arrow, labeled $v_{\mathrm{NF}}$ ) and the gas on the back side producing a component near the systemic velocity (open arrow, labeled $v_{\mathrm{NB}}$ ). The line of sight through the cone on the far-side of the disk may suffer heavy obscuration by dust in the disk. The gas on the front surface of the "far-side" cone will produce a component near systemic velocity (open arrow, labeled $v_{\mathrm{FF}}$ ), while the gas on the back surface will produce a strongly redshifted component (open arrow, labeled $v_{\mathrm{FB}}$ ). If the surface brightness of the gas drops rapidly as a function of distance from the nucleus, the component with velocity $v_{\mathrm{NB}}\left(v_{\mathrm{FF}}\right)$ will be brighter than the component with $v_{\mathrm{NF}}\left(v_{\mathrm{FB}}\right)$. See $\S \mathrm{IV} d$ and Table 6.

tions. However, this model also predicts that for the far side cone, the redshifted component should be brighter than the one near $v_{\text {sys }}$. This is definitely not the case in M82 (the component near $v_{\text {sys }}$ is brighter in both the far and near side cones).
The most plausible explanation of property $(\mathrm{h})$ within the context of a biconic outflow is that the surface brightness of the emitting material along the cone wall declines with distance from the nucleus. Along a given line of sight through a tipped cone, the kinematic component near $v_{\text {sys }}$ will always be produced by gas that is actually closer to the nucleus than gas producing the second kinematic component (see Fig. 19). For a surface brightness that drops like $r^{-n}$, it is easy to show that the ratio in the projected surface brightness of the component near $v_{\text {sys }}$ to the second component is given by $\left(r_{1} / r_{2}\right)^{1-n}$, where $r_{1}$ and $r_{2}$ are the true distances from the nucleus of the gas producing the two components. For example, in M82 the geometric parameters given in Table 6 imply that $r_{1} / r_{2} \approx 3$, so $n \approx 2$ would reproduce the typical 3:1 brightness ratio between the two kinematic components.

\section{e) Pressure Diagnostics in Superwinds}

\section{i) The Need for Ram Pressure Confinement}

We have shown in $\S$ III that the pressures in the optical emission-line clouds in the FIRG nebulae are very high relative to normal interstellar pressures, decline systematically with radii over scales of $1-10 \mathrm{kpc}$, and correlate with the IR luminosity of the galaxy. This strongly suggests that the gas pressures in these clouds are being regulated by some global mechanism relating to the starburst rather than by processes internal to the individual clouds. Indeed, in $\S$ IV $c$ we also showed that these pressures agreed well with the predictions of the superwind model in which the gas within the starburst (inside the wind's sonic radius) is confined by the thermal pressure of very hot $\left(T \approx 10^{8} \mathrm{~K}\right)$ thermalized stellar ejecta, and the gas at large radii is confined by the ram pressure of the superwind. Here we consider whether there is an alternative explanation for the high pressures and their global regulation. We will consider two obvious alternatives: (1) the measured pressures represent the radiation pressure of the central IR source, and (2) the emission-line gas is confined by the thermal pressure of an intercloud medium that is cool enough $(T<$ $10^{7} \mathrm{~K}$ ) to be gravitationally bound to the galaxy.

TABLE 6

PARAMETERS OF THE Biconic OUTFlow MODElS

\begin{tabular}{cccccc}
\hline \hline $\begin{array}{c}\text { Galaxy } \\
(1)\end{array}$ & $\begin{array}{c}V_{\text {near }} \\
(2)\end{array}$ & $\begin{array}{c}V_{\text {far }} \\
(3)\end{array}$ & $\begin{array}{c}\theta \\
(4)\end{array}$ & $\begin{array}{c}\phi \\
(5)\end{array}$ & $\begin{array}{c}V_{0} \\
(6)\end{array}$ \\
\hline NGC 253 $\ldots \ldots \ldots$ & -250 & +100 & $65^{\circ}$ & $15^{\circ}$ & 339 \\
M82 SE $\ldots \ldots \ldots \ldots$ & -300 & -50 & 62 & 40 & 317 \\
M82 NW $\ldots \ldots \ldots$. & 0 & +250 & 62 & 31 & 283 \\
NGC 4945 $\ldots \ldots \ldots$ & -500 & +50 & 78 & 33 & 525 \\
\hline
\end{tabular}

NoTES.-Key to cols. (2)-(6) follows:

Col. (2).-Average observed radial velocity $\left(\mathrm{km} \mathrm{s}^{-1}\right)$ relative to systemic of the blueshifted component of the double emission-line profiles.

Col. (3).-Average observed radial velocity $\left(\mathrm{km} \mathrm{s}^{-1}\right)$ relative to systemic of the redshifted component of the double emission-line profiles.

Col. (4).-Adopted opening angle of the cone, based on the measured extent of the region of double emission-line profiles (see Fig. 19 and the text).

Col. (5).-Predicted angle between the symmetry axis of the cone and the plane of the sky (see text and Fig. 19).

Col. (6).--Predicted outflow speed along the conic surface $\left(\mathrm{km} \mathrm{s}^{-1}\right)$. See text and Fig. 19. 
For the first case, we note that the measured pressures are about one order of magnitude greater than the radiation pressure associated with the starburst. Taking $P_{\mathrm{rad}}=L_{\mathrm{IR}} / 4 \pi r^{2} c$ implies that $r_{n 100}=500\left(L_{\mathrm{FIR}} / 10^{11} L_{\odot}\right)^{1 / 2} \mathrm{pc}$. Comparison of this relationship to the data in Figure 17 makes it clear that the FIRG nebulae are significantly overpressured relative to the radiation pressure.

To consider the second case, note that a hot medium in hydrostatic equilibrium in the potential well of a galaxy will have $T<10^{7} \mathrm{~K}$. We can calculate the minimum X-ray luminosity of this medium if it has a density high enough to confine the emission-line clouds. Taking a spherical distribution of hot gas with a radius of $1 \mathrm{kpc}, T=10^{7} \mathrm{~K}$, and $n \approx 0.2 \mathrm{~cm}^{-3}$ (appropriate to M82, NGC 253, NGC 1222, NGC 3079, NGC 4194 , and NGC 4945) yields an X-ray luminosity of $10^{41}$ ergs $\mathrm{s}^{-1}$ (exceeding the measured values for M82 and NGC 253 by about one order of magnitude). Increasing the radius to $3 \mathrm{kpc}$ (appropriate to NGC 1614, NGC 3256, NGC 3690, NGC 6240, Mrk 266, and Mrk 273) yields an X-ray luminosity of $3 \times 10^{42} \mathrm{ergs} \mathrm{s}^{-1}$ versus a measured X-ray luminosity of only $5 \times 10^{41} \mathrm{ergs} \mathrm{s}^{-1}$ for NGC 1614 .

Since a uniform density distribution with a sharp cutoff at the outer edge of the emission-line nebula is physically unrealistic, it is more instructive to compare the observed pressures and X-ray luminosities of the FIRG nebulae to those of the hot gas in giant elliptical galaxies. The estimated electron density in the hot phase in a giant elliptical is about $3-10 \times 10^{-3} \mathrm{~cm}^{-3}$ $\left(n_{e} T \approx 3-10 \times 10^{4} \mathrm{~cm}^{-3} \mathrm{~K}\right.$ ) at a radius of $2 \mathrm{kpc}$ (Thomas et al. 1987). We measure electron densities of about $100 \mathrm{~cm}^{-3}$ in the emission-line gas $\left(n_{e} T \approx 10^{6}\right)$ at radii of $0.6-5 \mathrm{kpc}$ in the FIRG nebulae. Thus, at about the same radius, the pressures in the FIRG nebulae are 10-30 times higher than in the giant ellipticals. Since the X-ray emissivity is proportional to the square of the density, we might expect the FIRGs to have X-ray luminosities 100-1000 times those of the ellipticals. However, the latter have typical X-ray luminosities of about $10^{41}$ ergs s $^{-1}$, compared to a range from $2 \times 10^{39}$ to $5 \times 10^{41}$ ergs s${ }^{-1}$ for the FIRGs (see Ward 1988).

Thus, it does not seem possible to confine the clouds in the FIRG nebulae with the thermal pressure of an intercloud medium that is cool enough to be in hydrostatic equilibrium in the galaxy's potential. Confinement by a much hotter $(T \gg$ $10^{7} \mathrm{~K}$ ) and less dense medium is consistent with the X-ray data, but then the sound speed in the hot phase will greatly exceed the escape velocity of the galaxy. Since we have shown previously that the radiative cooling time for such a fluid is far longer than its dynamical/sound-crossing time, even if this medium is initially static, it will quickly evolve into a wind (in fact, the superwind forms out of just such a hot fluid generated within the starburst).

\section{ii) Comparison to Predicted Pressure Profiles in Wind Models}

We have shown above that the characteristic pressures observed in the FIRG nebulae are consistent with the superwind model. In this section, we will consider the detailed shapes of the radial pressure profiles and compare them to the predictions of the superwind models. The two FIRGs with the best relevant data are M82 and NGC 3256 (which have nebulae with exceptionally high [S II] surface brightnesses and large angular sizes). These two galaxies represent both a low-power FIRG (M82 has $L_{\mathrm{IR}} \approx 10^{44} \mathrm{ergs} \mathrm{s}^{-1}$ ) and a high-power FIRG ( NGC 3256 has $L_{\mathrm{IR}} \approx 10^{45} \mathrm{ergs} \mathrm{s}^{-1}$ ). Figure 20 is a plot of log $n_{e}$ versus $\log r$ for M82 and NGC 3256 in which we have "slid" NGC 3256 over by 0.45 dex in radius (both $r_{n 100}$ and $r_{*}$ are 0.45 dex larger in NGC 3256). Consideration of this figure (supplemented by Fig. 13 for the other FIRGs) leads to a number of interesting conclusions:

1. The radial pressure profiles for the two galaxies in Figure 20 are amazingly similar apart from the larger radial scales in NGC 3256. Clearly, the same mechanism is regulating the physical conditions in the two galaxies.

2. The radial pressure profiles in M82 and NGC 3256 steepen markedly as the radius increases. While the data for the other 10 FIRGs in Figure 13 are less detailed, six more cases (NGC 253, NGC 1614, NGC 3690, NGC 4194, Mrk 273, and possibly NGC 3079 ) exhibit similar behavior. For these cases, as for M82 and NGC 3256, the pressure profiles at large radii are consistent with the $r^{-2}$ dependence expected for ram pressure. However, in none of the 12 FIRGs in Figure 13 does the pressure profile follow an $r^{-2}$ law for small radii. This is as expected for a superwind driven by a starburst whose angular size is much greater than the size of the seeing disk. However, it is not consistent with a superwind driven by an AGN (where the injection region is much less than the seeing disk): in such a case, the pressure would follow an inverse square law all the way in to the central nucleus. Thus we conclude that not only do the magnitudes of the measured pressures agree with the predictions of the superwind model, but so do the shapes of the radial pressure profiles. They are shallow at small radii within the region of momentum injection where static thermal pressure dominates. They then steepen to $r^{-2}$ at large radii where the ram pressure of the superwind dominates.

3. The profile shapes in M82 and NGC 3256 match the prediction of the model of $\mathrm{CC}$ for a freely expanding superwind at small radii (less than $\frac{1}{3} r_{*}$ and large radii (greater than $2 r_{*}$ ). However, at intermediate radii the observed densities and pressures are systematically lower than the predictions (by a factor of about 2 at $r_{*}$ ). We believe that this discrepancy between the observed and predicted profile shapes might be due to the simplified model adopted by $\mathrm{CC}$ for the region of momentum injection (namely, an injection rate per unit surface area that was uniform inside $r_{*}$ and zero outside). That is, if the SFR (and thus the resulting rate of momentum injection ) per unit surface area were instead taken to be a smoothly falling function of radius, profiles more like those observed would result.

4. There is a correspondence between the kinematics of the gas and the pressure profiles in the nearby edge-on FIRGs. Note that $r_{*}$ is not only the outer boundary of the region within which the momentum injection occurs but is also near the wind's sonic point, and hence marks the transition to rapid outflow. It is intriguing in this regard that the regions along the galaxy minor axes exhibiting split lines in M82, NGC 253, NGC 3079, and NGC 4945 are found at radii greater than $100-500 \mathrm{pc}$ from the nucleus (see $\S$ III). It therefore appears that these kinematic structures are found beyond the sonic point of the wind (just as expected if they represent entrained, accelerated material). 


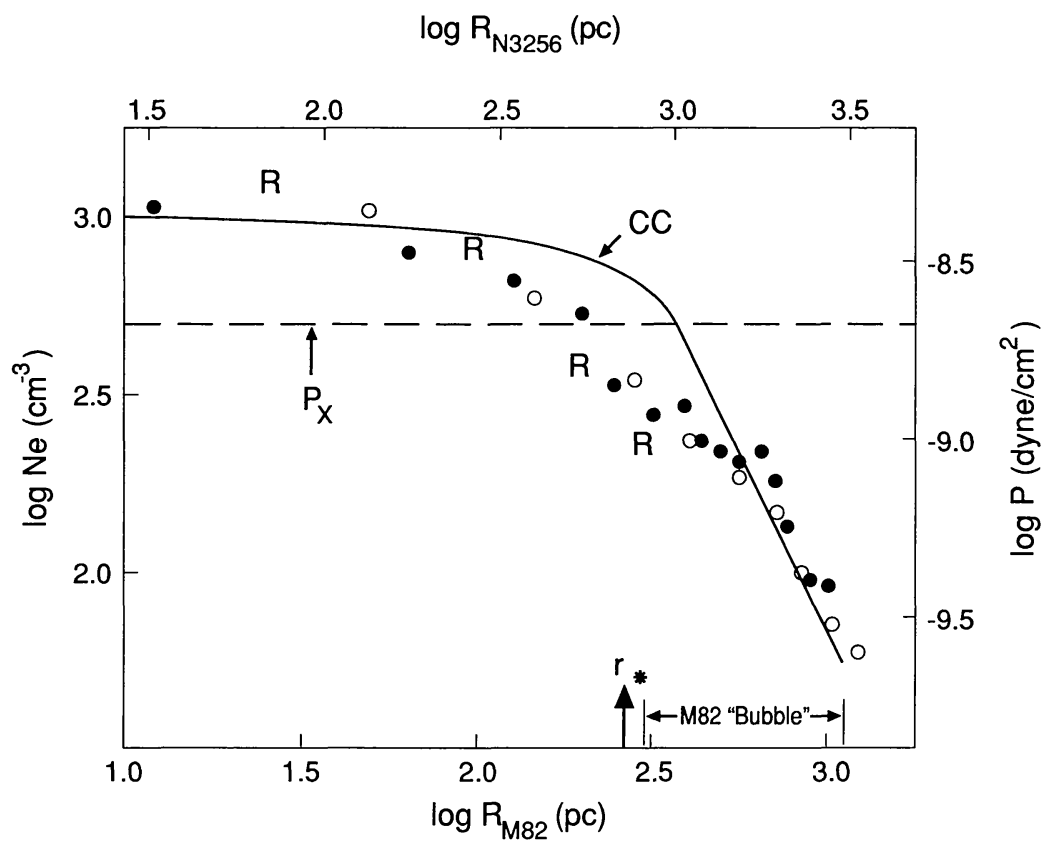

FIG. 20.-A plot of the radial density/pressure profile in M82 (filled circles) overlain with the same for NGC 3256 (open circles). The plot for NGC 3256 has been shifted by 0.45 dex in radius with respect to M82: the radial scale in M82 (NGC 3256) is indicated on the lower (upper) horizontal axis. The shapes of the profiles are remarkably similar in the two galaxies, and the larger radial scale associated with the more luminous starburst in NGC 3256 is predicted by the superwind model. The radius of the starburst (the mass/energy injection region) is denoted by $r_{*}$, which is also roughly the sonic radius for the wind. The solid curve is the shape of the pressure profiles predicted by the wind model using the parameters given in Table 5 (these were selected to match the pressures for $r \ll r_{*}$ and $r \gg r_{*}$ ). The predicted pressures are high between $r \approx 0.5-2 r_{*}$. We have also indicated the location of the region of split lines in the M82 nebula ("M82 bubble"), which lies just outside $r_{*}$. The average pressure in the X-ray gas within a radius of about $1 \mathrm{kpc}$ of the nucleus of M82 is indicated by an arrow labeled $P_{\mathrm{x}}$ using the results of Schaaf $e t$ al. (1989) for a filling factor of unity. Pressures in the radio plasma at four locations along the minor axis of M82 are indicated by " $R$." The shape of the radial pressure profile in the radio plasma was computed from the idealized model in Seaquist, Bell, and Bignell (1985). We have displayed radio pressures that are a factor of 5 higher than the pressures implied by the standard minimum energy assumptions in order to facilitate comparison of the shapes of the radio and optical pressure profiles.

\section{iii) Comparison to $X$-Ray and Radio Pressures}

In the case of M82, we can compare the pressures in the warm gas ( $T \approx 10^{4} \mathrm{~K}$, as measured with the [S II] lines) to pressures in the hot phase ( $T \approx 10^{8} \mathrm{~K}$, probed with X-rays), cold phase (the molecular gas), and relativistic phase (the radio-emitting synchrotron plasma).

For the hot phase, Schaaf et al. (1989) derive a mean electron density of $0.085 \mathrm{ff}^{-1 / 2} \mathrm{~cm}^{-3}$ within a radius of about 1.5 $\mathrm{kpc}$ of the nucleus ( $f f$ is the filling factor of the hot gas, which is predicted by the wind-blown shell models to be in the range $0.1-1)$. The corresponding pressure is then about $2 \times 10^{-9}$ $\mathrm{ff}^{-1 / 2} T_{8}$ dynes $\mathrm{cm}^{-2}$, where $T_{8}$ is the temperature in units of $10^{8} \mathrm{~K}\left(T_{8} \approx 0.6-2.2\right.$ according to Schaaf et al. 1989). This can be compared to the pressures we have estimated for the optical emission-line gas which range from about $4 \times 10^{-9}$ dynes $\mathrm{cm}^{-2}$ for gas with $r<100 \mathrm{pc}$ to $4 \times 10^{-10}$ dynes $\mathrm{cm}^{-2}$ at $r \approx 1000 \mathrm{pc}$. Thus, the pressures in the hot and warm phases appear to be in satisfactory agreement.

Schaaf et al. (1989) have also used the radio data of Kronberg, Biermann, and Schwab (1985) to calculate a minimum pressure in the central $(400 \times 70 \mathrm{pc})$ radio synchrotron disk of M82 of $\approx 10^{-9}$ dynes $\mathrm{cm}^{-2}$. Thus, the pressure in the radio plasma is at least $25 \%$ of the optically derived pressure. Seaquist, Bell, and Bignell (1985) have mapped the nonthermal radio emission out to distances of about $500 \mathrm{pc}$ above and below the galaxy disk in M82 (i.e., within the inner region of the superwind nebula). If we adopt their slab model of the radio source and assume that the ratio of actual pressure to minimum pressure is constant with height above the central disk, we find that the pressure in the radio plasma drops smoothly with the radius in a manner that is similar to the pressure drop in the emission-line gas. The best fit to the pressures measured in the optical are achieved if the pressures in the radio plasma are about 5 times the minimum-energy pressures (see Fig. 20).

Finally, Turner, Martin, and Ho (1990) have investigated the molecular gas in the M82 starburst region and estimate a pressure of about $10^{-9}$ dynes $\mathrm{cm}^{-2}$. Within the uncertainties, this is also consistent with the pressures measured in the optical emission-line gas and the $\mathrm{X}$-ray and radio-synchrotron plasmas.

\section{f) Radiation from Superwinds}

i) X-Rays

There are several possible sources of thermal X-rays from a superwind. When the wind is in the radiative phase, CMW and MM show that the significant X-ray emission will arise from wind material that has been heated in an internal shock located upstream from its contact discontinuity with the swept-up 
shell material. TI have also calculated the X-ray emission from this material in their numerical model of the M82 superwind. The values they derive agree well with the simple analytic results of CMW. Scaling the relations in CMW and MM for the postshock temperature and X-ray luminosity of the shocked wind material, we find

$$
\begin{aligned}
& T_{x}=6 \times 10^{7} n_{0}^{2 / 35} d E / d t_{43}^{8 / 35} t_{6}^{-6 / 35} \mathrm{~K} \\
& n_{x}=0.12 d E / d t_{43}^{6 / 35} n_{0}^{19 / 35} t_{6}^{-22 / 35} \mathrm{~cm}^{-3} \\
& L_{x}=7 \times 10^{39} d E / d t_{43}^{37 / 35} n_{0}^{18 / 35} t_{6}^{13 / 35} \mathrm{ergs} \mathrm{s}^{-1}
\end{aligned}
$$

For the values of $n_{0}, d E / d t_{43}$, and $t_{6}$ which we have estimated for the superwinds (see Table 5), we predict $\log L_{x} \approx$ $39.5-41.5$, with the larger values appropriate to the more powerful FIRGs. We have estimated in $\S \mathrm{IV} c$ (iii) that $d E / d t$ is a few percent of the bolometric (taken to be the IR) starburst luminosity. Equation (15), then, predicts that X-ray luminosity from the superwind will be only about $10^{-5}-10^{-4}$ of $L_{\mathrm{IR}}$. There are five galaxies in our sample with published X-ray luminosities, and these range from $5 \times 10^{39}$ to $5 \times 10^{41}$ ergs $\mathrm{s}^{-1}$. We can add NGC 3628 to this sample, since Fabbiano, Heckman, and Keel (1990) have described the optical and $\mathrm{X}$-ray evidence for a superwind in this edge-on FIRG. As summarized in Table 7, the values of $\log \left[L_{x} / L_{\text {FIR }}\right]$ are typically -4 for these six superwind galaxies. Thus, both the magnitude of the X-ray emission and its relationship to the inferred starburst luminosity are in agreement with the models. Moreover, the temperature and density of the X-ray gas in M82 agree well with the predictions of equations (13) and (14) (Schaaf et al. 1989 ). In a related vein, Ward (1988) has recently shown that starburst galaxies have X-ray powers that scale with the inferred Lyman continuum luminosity of the starburst.

The most convincing evidence that much of the X-rays arise in the wind is the morphological correspondence between extranuclear (spatially resolved) X-ray emission and the optical emission-line filaments along the minor axes of M82, NGC 253, and NGC 3628 (Fabbiano 1988; Watson, Stanger, and Griffiths 1984; Fabbiano and Trinchieri 1984; Fabbiano, Heckman, and Keel 1990). The X-ray emission is also known

TABLE 7

X-RAY Properties of SUPERWind GalaXies

\begin{tabular}{cccc}
\hline \hline $\begin{array}{c}\text { Galaxy } \\
(1)\end{array}$ & $\begin{array}{c}\log L_{\mathrm{x}} \\
(2)\end{array}$ & $\begin{array}{c}\log L_{\mathrm{IR}} \\
(3)\end{array}$ & $\begin{array}{c}\log \left(L_{\mathrm{x}} / L_{\mathrm{IR}}\right) \\
(4)\end{array}$ \\
\hline NGC $253 \ldots \ldots$ & 40.0 & 44.1 & -4.1 \\
NGC $1614 \ldots \ldots$ & 41.3 & 45.0 & -3.7 \\
M82 $\ldots \ldots \ldots \ldots$ & 40.4 & 44.1 & -3.7 \\
NGC $3079 \ldots \ldots$ & 40.0 & 44.1 & -4.1 \\
NGC $3628 \ldots \ldots$ & 40.2 & 44.2 & -4.0 \\
Arp 220...... & 41.4 & 45.7 & -4.3 \\
\hline
\end{tabular}

NOTES.-Key to cols. (2) and (3) follows:

Col. (2). - The log of the global keV (typically $0.2-4 \mathrm{keV}$ ) X-ray luminosity in ergs s ${ }^{-1}$. See Ward 1988 and references therein, except for NGC 3079 (Fabbiano 1989, private communication) and NGC 3628 (Fabbiano, Heckman, and Keel 1990).

Col. (3). - The log of the total IR luminosity in ergs s ${ }^{-1}$ (see Table 1). to be spatially extended with a size comparable to the emission-line bubbles in Arp 220 (Eales and Arnaud 1988).

Ambient material that is shock heated by the superwind can, in principle, provide an additional source of X-rays. Shock velocities of at least $10^{3} \mathrm{~km} \mathrm{~s}^{-1}$ are required to produce hot $\left(T>10^{7} \mathrm{~K}\right), \mathrm{X}$-ray emitting gas. For the ram pressure of the superwind to drive shocks with velocities this high, the ambient densities must be less than a few $\times 10^{-2} \mathrm{~cm}^{-3}$. The minimum cooling time for such shocked material will be about $10^{8}$ $\mathrm{yr}$ (for a postshock temperature and density of $10^{7} \mathrm{~K}$ and 0.1 $\mathrm{cm}^{-3}$, respectively). Since this time scale exceeds the wind's dynamical lifetime by about one order of magnitude, it is unclear whether shocked ambient material is a significant source of thermal X-rays in superwinds.

One possible observational test to discriminate between the two above mechanisms would be to measure the temperature of the hot gas. Gas heated by the internal shock in the wind should have a temperature of order $10^{8} \mathrm{~K}$-see equation 13 . In contrast, the shock-heated ambient clouds with the shortest cooling times will have the highest densities and lowest temperatures $\left(T<10^{7} \mathrm{~K}\right)$. In M82 (the best-studied case by far), the $\mathrm{X}$-ray temperature is given by Schaaf et al. (1989) as $6 \times 10^{7}$ to $2.2 \times 10^{8} \mathrm{~K}$. These high temperatures favor an internal wind shock in M82.

\section{ii) Optical Emission Lines}

The FIRGs we have discussed above are strong sources of optical line emission, and this emission arises in large-scale (several $\mathrm{kpc}$ to several tens of $\mathrm{kpc}$ ) nebulae rather than being purely nuclear. These properties are typical of galaxies that are powerful in the far-IR (Armus, Heckman, and Miley 1990). We have suggested above that these nebulae may be "powered" by shocks driven into ambient material by superwinds. The relative intensities of the strong lines are consistent with this, as are the high electron temperatures measured in the $\mathrm{O}$ III zone in some of the nebulae (see $\S$ III $b$ above). Here we wish to explore this idea in terms of the energetics of superwinds and shocks.

First, it is easy to show that the cooling time in the gas observed optically is much shorter than the superwind dynamical time (i.e., the shocks driven into the ambient gas should be strongly radiative). Using the shock models of BDT and the densities we derived from the [S II] lines, we find that the postshock cooling time ranges from a few hundred years to about $10^{4} \mathrm{yr}$ for shock velocities from $100-300 \mathrm{~km} \mathrm{~s}^{-1}$. The radiative cooling times become comparable to the dynamical times of $\approx 10^{7}$ yr only if the shock velocities exceed about 800 $\mathrm{km} \mathrm{s}^{-1}$.

We can also show that superwinds appear energetically capable of powering the nebulae. Typical total $\mathrm{H} \alpha$ luminosities (uncorrected for dust extinction in the FIRGs) range from $\log L_{\mathrm{H} \alpha} \approx 40-42$ (cgs units). The extinctions in the nebulae are estimated from the measured Balmer decrements to be typically factors 3-10 for $\mathrm{H} \alpha$ (discussed in detail in Armus, Heckman, and Miley 1990). The models of either shock-excited or photoionized gas that best reproduce the observed emission-line ratios yield a total emission-line luminosity of about 30 times the $\mathrm{H} \alpha$ luminosity. Thus, the FIRG nebulae 
have total, extinction-corrected emission-line luminosities of $\log L_{\text {neb }} \approx 42.2-44.2$. The emission-line luminosities correlate with the IR luminosity (Armus, Heckman, and Miley 1990), with the typical extinction-corrected ratios of $L_{\text {neb }} / L_{\mathrm{IR}} \approx$ $0.01-0.1$.

We can compare these luminosities to the estimated kinetic energy flux in a superwind $(d E / d t)$. The wind models predict that $d E / d t$ will be several percent of $L_{\mathrm{TR}}$, and this also agrees with our observational estimates of $d E / d t$. Since $L_{\text {neb }} \approx 1 \%-$ $10 \%$ of $L_{\mathrm{IR}}, d E / d t \approx L_{\text {neb }}$ in typical cases. Thus, it is energetically possible for superwinds to power the emission-line nebulae, but only marginally so (the efficiency with which the wind's energy must be converted into emission lines is uncomfortably large).

A further check on the viability of shock excitation is to calculate the rate at which material must be shocked in order to account for the observed $\mathrm{H} \alpha$ luminosity. Shock models indicate that each swept-up (shocked) $\mathrm{H}$ atom will emit about one Balmer-line photon for shock velocities in the range 90-160 $\mathrm{km} \mathrm{s}^{-1}$ (Shull and McKee 1979), but about 5 Balmer photons per proton for shock speeds of $\approx 300 \mathrm{~km} \mathrm{~s}^{-1}$ (Kang and Shapiro (1989). Since the outflow speeds inferred from our biconic outflow models are $\approx 300 \mathrm{~km} \mathrm{~s}^{-1}$, adopting this value for the typical shock velocity then requires that matter is being shocked at a rate

$$
d M / d t_{\text {shock }} \approx 38 L_{\mathrm{H} \alpha, 40} M_{\odot} \mathrm{yr}^{-1}
$$

where the $\mathrm{H} \alpha$ luminosity is in units of $10^{40} \mathrm{ergs} \mathrm{s}^{-1}$. Thus (for example) excitation of the entire M82 nebula by shocks would require that $d M / d t \approx 760 M_{\odot} \mathrm{yr}^{-1}$. Over either the dynamical lifetime of the wind or the duration of a starburst $\left(\approx 10^{7} \mathrm{yr}\right)$, this implies an unreasonably large amount of shocked/entrained matter (nearly $10^{10} M_{\odot}$ ).

Finally, the shock models of BDT yield an $\mathrm{H} \alpha$ surface brightness of $\approx 3 \times 10^{39} \mathrm{ergs} \mathrm{s}^{-1} \mathrm{kpc}^{-2}$ for a shock velocity of $340 \mathrm{~km}$ $\mathrm{s}^{-1}$ and for densities similar to those observed in the FIRG nebulae. Taking (as an example) the M82 nebula to have a cylindrical geometry with a surface area of $\approx 5 \mathrm{kpc}^{2}$, then, yields a predicted $\mathrm{H} \alpha$ luminosity of $\approx 1.5 \times 10^{41} \mathrm{ergs} \mathrm{s}^{-1}$ (less than $10 \%$ of the observed total).

We therefore conclude that shock heating is probably not viable as the sole excitation mechanism in FIRG nebulae. As an alternative, we consider the suggestion made by Armus, Heckman, and Miley (1989) that the excitation mechanism changes over from photoionization by the starburst in/near the nucleus to shock heating by superwinds in the outer parts of the nebulae. This suggestion was motivated in part by the pronounced radial gradient they observed in the relative strength of the [O $\mathrm{I}$ ] $\lambda 6300$ line in M82. Let us adopt this hypothesis and take only the gas in M82 at $R>300$ pc to be shock-heated. This is where the spectrum is clearly LINERlike, is in the region where the wind's ram pressure is dominant, and is where double emission-line profiles are found. The extinction-corrected $\mathrm{H} \alpha$ luminosity of this outer component is only about $2 \times 10^{40} \mathrm{ergs} \mathrm{s}^{-1}$, or about $10 \%$ of the total (see $\S$ III $c$ above). This more modest luminosity then requires that only about $10 \%$ of the superwind's mechanical energy be converted via shocks into line emission, implies that $d M / d t_{\text {shock }}$ need only be $\approx 76 M_{\odot} \mathrm{yr}^{-1}$, and is in good agreement with the $\mathrm{H} \alpha$ luminosity predicted by the BDT model with a shock speed of $340 \mathrm{~km} \mathrm{~s}^{-1}$.

Could all of the gas be photoionized by the central starburst? The principal problem with this idea is that the LINER-type spectra in the outer parts of the nebulae cannot be produced by normal O stars. As we emphasized in $\S$ III $b$ above, the spectra of the FIRG nebulae differ strongly from the spectra of ordinary disk $\mathrm{H}$ II regions. Furthermore, it is difficult to understand even theoretically how to produce the LINER-type spectra of the FIRGs using ordinary hot, massive stars. Comparison to the photoionization models of Evans and Dopita (1985) is instructive in this regard. They constructed a grid of models with two free parameters, the temperature of the ionizing star $\left(T_{*}\right)$ and the ionization parameter $U$ (a meaure of the ratio of the densities of ionizing photons to electrons in the emissionline cloud). Thus, for a cloud located a distance $r$ from the ionization source, we can write $U=Q_{\mathrm{Ly}} /\left(4 \pi r^{2} c n_{\mathrm{e}}\right)$. In M82 and NGC 253 (for example) $Q_{\mathrm{Ly}} \approx 2-3 \times 10^{53} \mathrm{~s}^{-1}$. We measure $n_{e} \approx 100 \mathrm{~cm}^{-3}$ at $r=800 \pm 50 \mathrm{pc}$ for these two galaxies. Thus, the outer parts of the nebulae should have $\log U \approx-3.0$. The $\left[\mathrm{O}_{\mathrm{I}}\right] \lambda 6300$ line has a strength that is most sensitive to the value of $T_{*}$ because strong [ $\mathrm{O}_{\mathrm{I}}$ ] emission requires an abundant supply of high-energy photons to penetrate and heat the neutral zone in the emission-line clouds. The Evans and Dopita (1985) models for $\log U=-3.0$ give values for the flux ratio $\log (\lambda 6300 / \mathrm{H} \alpha)$ of $-2.2,-1.9,-1.5,-1.3$, and -1.2 for $T_{*}$ of $37,000 \mathrm{~K}, 40,000 \mathrm{~K}, 45,000 \mathrm{~K}, 50,000 \mathrm{~K}$, and $56,000 \mathrm{~K}$, respectively. For comparison, $\log (\lambda 6300 / \mathrm{H} \alpha) \approx-1.0$ at this radius in both M82 and NGC 253.

The above implies that the starburst must be characterized by $T_{*}>56,000 \mathrm{~K}$ in order that the M 82 and NGC 253 nebula have strong enough [O I] emission. In contrast, Evans and Dopita (1985) find that $\mathrm{H}$ II regions in external galaxies require $T_{*} \approx 41,000 \mathrm{~K}$, with little variation. McCall, Rybski, and Shields (1985) find that $T_{*}$ is a function of metal abundance and ranges from $39,000 \mathrm{~K}$ to $47,000 \mathrm{~K}$ as the metal abundance drops from 4 to 0.1 times solar. Values of $T_{*} \geq$ $56,000 \mathrm{~K}$ are inferred only for $\mathrm{H}$ iI regions in extremely metaldeficient ( $5 \%$ solar metallicity) dwarf galaxies (Campbell 1988). Such objects are characterized by an almost total lack of dust (presumably due to the low metal abundances), quite the opposite of the dust-shrouded FIRGs. It is therefore very unlikely that the FIRG nebulae are photoionized by the starburst, unless the starburst is dominated by very unusual stars (e.g., the "warmers" hypothesized by Terlevich and Melnick 1985).

While photoionization by an AGN could in principle produce spectra that resemble those of FIRG nebulae ( see Ferland and Netzer 1983), this idea can be excluded on the basis of ionization balance arguments, as we now show. The shape of a typical measured radial $n_{e}$ profile (discussed above) implies that a point source of ionizing photons will lead to an ionization parameter, $U$, that drops sharply with increasing radius in the inner region of the nebula where the radial $n_{e}(r)$ profile is relatively flat. Such a drop in $U$ should produce a strong radial variation in line ratios that are sensitive to $U$ (e.g., [O III] $\lambda 5007 / \mathrm{H} \beta)$. For the seven FIRGs in our sample with measured [O III]/H $\beta$ (NGC 1222, NGC 1614, M82, NGC 3690, 
NGC 4194, Mrk 266, and Mrk 273), the line ratio does not change strongly with radius (changes less than a factor of 2 , with an equal number of cases of radially rising and falling $[O$ III ] $/ \mathrm{H} \beta)$. This, then, rules out photoionization by an AGN or by any other photoionization source whose size is smaller than a few hundred parsecs.

To conclude, the most plausible mechanism for heating the emission-line gas in superwind galaxies is one in which the bright near-nuclear gas is photoionized by the central starburst, while the gas at larger radii is shock-excited by the superwind.

\section{g) The Dynamical State of Superwinds: Evidence for Blowout?}

In order to assess the astrophysical implications of superwinds, it is crucial to determine whether they are able to break free of the ISM of the starburst galaxy and thereby propel mass, energy, and metals out into the intergalactic medium. In the parlance of the theoretical work on winds, we aim to determine whether superwinds reach the blowout phase. We believe that at least some of the objects discussed in this paper are superwinds in the blowout phase, for the following reasons:

1. The theoretical argument given in $\S$ IV $b$ implies that superwinds have such high mechanical energy fluxes that they should be able to blow out of any reasonable galaxian exponential atmosphere (see also Norman and Ikeuchi 1989).

2. The X-ray plume along the minor axis of M82 extends out to a radius of at least $8 \mathrm{kpc}$ from the nucleus (well beyond the region of double emission-line profiles), and the X-ray surface brightness falls as $r^{-3}$, which is consistent with a freely expanding isothermal wind (Fabbiano 1988). These results are very difficult to understand if the wind were still confined inside a bubble, since the X-rays should then arise in an isobaric annulus of thermalized wind fluid located just inside the optically radiating shock being driven into the ambient gas (see Fig. 1).

3. The morphologies of the optical emission-line nebulae in the many of the FIRGs suggest that the gas is located along the walls of cones or cylinders that are open at their outer extremities (i.e., they are limb-brightened on their sides but not at their "tops"). The nebulae in NGC 253 (MVH), NGC 6240 (HAM), and NGC 3079 (Ford et al. 1986; Armus, Heckman, and Miley 1990) are particularly good examples of the "ruptured bubble" morphology predicted for a superwind in the blowout phase. The faint quasi-radial emission-line filaments in the halos of NGC 3628 (Fabbiano, Heckman, and Keel 1990) and NGC 3079 (Fig. 7) are further examples. The NGC 4945 and Arp 220 nebulae-_bubbles" that are limb-brightened on their outer edges-are counterexamples.

4. Most of the radial pressure profiles derived from our spectra drop rapidly at large radii. This is consistent with the ram pressure of a freely expanding wind, but is inconsistent with a wind-driven bubble that is still confined by the ambient medium (the bubble's interior should be roughly isobaric in this case).

\section{IMPLICATIONS OF SUPERWINDS}

\section{a) Mass, Metal, and Energy Injection Rates of Superwinds}

In $\S \mathrm{IV}$, we concluded that a typical superwind is characterized by mass and energy flow rates of $1-100 M_{\odot} \mathrm{yr}^{-1}$ and $3 \times$
$10^{42}-3 \times 10^{44} \mathrm{ergs} \mathrm{s}^{-1}$, respectively. Taking the inferred superwind lifetimes of about $10^{7} \mathrm{yr}$, then, implies total masses and energies of $10^{7}-10^{9} M_{\odot}$ and $10^{57}-10^{59}$ ergs, respectively. If the superwind achieves the blowout phase, then all its mass and much of its energy can escape into the IGM. As we described earlier, the superwind might entrain significant amounts of ambient gas which it may be able to propel out of the galaxy. The maximum amount of ambient material that could be ejected by the wind is enormous (essentially the entire ISM of the galaxy):

$$
M_{\mathrm{ej}, \max } \approx 10^{10}\left(E_{\text {wind }} / 10^{58} \mathrm{ergs}\right)\left(v_{\mathrm{esc}} / 450 \mathrm{~km} \mathrm{~s}^{-1}\right)^{-2} M_{\odot}
$$

In most cases, the observed velocities of the optically emitting material in the FIRG nebulae are similar to the local escape velocity (few hundred $\mathrm{km} \mathrm{s}^{-1}$ ), so it is unclear how much of this entrained material actually escapes the galaxy.

A more instructive way to assess the possible cosmological significance of superwinds is to estimate the global rate at which they inject mass, energy, and metals into the IGM at the current epoch (we remind readers that our adopted cosmology is $H_{0}=75 \mathrm{~km} \mathrm{~s}^{-1} \mathrm{Mpc}^{-1}$ and $q_{0}=0$ ). To make this estimate, we assume that the outflow rates in a superwind driven by a starburst are related to the SFR, which is in turn related to the IR luminosity. We will therefore compute the IR energy density in the local universe due to galaxies and then relate this to the desired superwind parameters scaling from wellstudied prototype superwind/starburst galaxies like M82 and NGC 253.

The SFR will not be strictly proportional to the IR luminosity because some of the emission comes from interstellar clouds heated by the diffuse radiation field of the old stellar population (the "IR cirrus" component). This component is characterized by cool dust temperatures and is dominant in galaxies with low IR luminosities. The second, warmer IR component associated with star-formation becomes dominant in galaxies with high IR luminosities (see the review by Soifer, Houck, and Neugegauer 1987, and references therein). It is also clear that the superwind rate is not strictly proportional to the global SFR in all galaxies. Indeed, we have argued throughout this paper that the superwind phenomenon is associated with galaxies having very high SFRs $\left(10-100 M_{\odot} \mathrm{yr}^{-1}\right)$ on circumnuclear $(100-1000 \mathrm{pc})$ scales. This again suggests that we should include in our estimation of the local superwind rate only those galaxies with the extreme IR properties of the galaxies we have investigated in this paper (namely high IR luminosities, high IR-to-visible luminosity ratios, and high dust temperatures).

We will, therefore, estimate the local IR energy density in three ways chosen to isolate starburst/superwind galaxies. First, we will consider only galaxies with luminosities above the "knee" in the IR luminosity function at about $10^{44} \mathrm{ergs} \mathrm{s}^{-1}$ (see Soifer et al. 1987). All the galaxies we have discussed above meet this criterion (Table 1 ). In fact, the three closest galaxies in the universe with $L_{\mathrm{IR}} \geq 10^{44} \mathrm{ergs} \mathrm{s}^{-1}$ are NGC 253, M82, and NGC 4945, all of which show clear evidence for superwinds. Second, we will select only those galaxies whose IR luminosities are large relative to their optical luminosity 
$\left(L_{\mathrm{IR}} / L_{B}>2\right)$. Again, all the galaxies in our sample meet this criterion ( see Table 1). Finally, we will consider only those galaxies with "warm" IR spectra (flux density at $60 \mu \mathrm{m}>50 \%$ of the flux density at $100 \mu \mathrm{m}$ ). All of our galaxies meet this criterion, except for NGC 4945 where the ratio is $42 \%$. The IR luminosity function in Soifer et al. (1987) yields a total IR luminosity density from galaxies of about $9 \times 10^{7} \mathrm{~L}_{\odot} \mathrm{Mpc}^{-3}$, of which $\approx 40 \%$ comes from above the "knee." Similarly, Bothun, Lonsdale, and Rice (1989) find that about one-third of the total luminosity comes from galaxies with $L_{\mathrm{IR}} / L_{B}>2$, and about half from galaxies with "warm" IR emission (as defined above). We therefore assume that the local IR energy density associated with starburst/superwind galaxies is $3.6 \times$ $10^{7} L_{\odot} \mathrm{Mpc}^{-3}$.

We then take the mass-injection rate given by equation (11) for the wind material, yielding a global mass-injection rate of $1.5 \times 10^{-3} M_{\odot} \mathrm{yr}^{-1} \mathrm{Mpc}^{-3}$ in superwind material. Multiplying by $1 / H_{0}$, we estimate that the total mass-injection rate is about $\approx 2 \times 10^{7} M_{\odot} \mathrm{Mpc}^{-3}$, or an average of $\approx 2 \times 10^{9} M_{\odot}$ per Schecter $L_{*}$ galaxy (using the average galaxy density given in de Lapparent, Geller, and Huchra 1988). Taking mass fractions for the metals of $25 \%$ in the wind material (Maeder 1990) implies that the mass of ejected metals is $\approx 5 \times 10^{8} M_{\odot}$ per $L_{*}$ galaxy, approximately equal to the total metals in an $L_{*}$ galaxy with $M / L=10$ and solar abundances. These figures all ignore any entrained material that has been ejected by the wind. The maximum mass of such ejected material is $\approx 10^{11} M_{\odot}$ per $L_{*}$ galaxy (eq. [17]). Repeating the above calculations for the energy injection by superwinds (using eq. [11]), we get $\approx 10^{57}$ ergs $\mathrm{Mpc}^{-3}$, and thus the equivalent of $\approx 10^{59} \operatorname{ergs}$ per $L_{*}$ galaxy (approximately the typical gravitational binding energy of a galaxy).

We conclude that superwinds appear to be globally significant in terms of their energetics and the cycling of matter (particularly metals), even without any correction for the expected strong cosmological evolution in the superwind rate. For example, if the starburst luminosity (and hence superwind rate) evolves as $(1+z)^{\mathrm{x}}$, where $x \approx 3-4$ (as suggested by faint source counts-Hacking, Condon, and Houck 1987; Lonsdale and Hacking 1989), and this evolution extends to $z_{\max } \approx 3$, then the total amounts of injected mass, metals, and energy would increase by about one order of magnitude relative to the above estimates.

\section{b) Heating and Enriching the Intergalactic Medium}

The intracluster medium (ICM) in rich clusters of galaxies contains approximately as much mass and metals as the galaxies themselves (e.g., Forman and Jones 1982; Sarazin 1986, and references therein). The mechanism by which such large quantities of metal-enriched material (and hence material which has been cycled through stars) could be transported out into the ICM is unclear. Ram pressure stripping of the ISM of cluster galaxies is apparently operating at the present epoch (e.g., van Gorkum and Kotanyi 1985), but requires that a substantial ICM already be present. Alternatively, the ICM could have been enriched by wind-driven mass loss from galaxies, a concept that has been explored theoretically by several different authors (e.g., Larson and Dinerstein 1975; De Young 1978).
We have calculated above that the amount of metals expelled by superwinds might be at least comparable to the amount of metals contained in galaxies. This suggests that superwinds have played a very important (and perhaps dominant) role in the transport of metals from galaxies into the ICM. The metal abundance in the superwind fluid itself would be $\approx 15$ times solar for a Salpeter IMF with an upper mass cutoff of $100 M_{\odot}$ (Maeder 1990). Since the ICM has a metal abundance that is $30 \%-100 \%$ solar (see Sarazin 1986, and references therein), the metals supplied by superwinds must have been highly diluted (either by the IGM or by interstellar material entrained and ejected by the superwind). Thus, while the metals in the ICM could be supplied by superwinds, the bulk of the mass $(\approx 95 \%)$ of the ICM could have a primordial origin (e.g., Gunn and Gott 1972). In this case, there should be an inverse correlation between the ICM mass (normalized to the total mass of the stellar population in the cluster) and the metal abundance of the ICM. Such a correlation may in fact be present (David et al. 1990).

Heating of the ICM by superwinds may also be important. If superwinds are responsible for the metal enrichment of the ICM, the heating provided by the thermalization of their kinetic energy would give the ICM a greater effective temperature than the galaxies. As noted by White (1990), this heating should be especially important in clusters with low velocity dispersions $\left(\sigma \leq 500 \mathrm{~km} \mathrm{~s}^{-1}\right.$ ). White (1990) investigates the parameter $\beta=\mu m_{\mathrm{H}} \sigma^{2} / k T_{\mathrm{ICM}}$, a measure of the ratio of the specific energies in the galaxies and the ICM. He finds that clusters with low velocity dispersions have $\beta$ significantly smaller than unity, while clusters with high velocity dispersions have $\beta \approx 1$. He therefore concludes that the ICM has been enriched and heated by galactic winds. Within the context of this type of model, his best fit to the $\sigma$ versus $T_{\text {ICM }}$ relation for clusters implies that $w^{1 / 2} v_{\text {wind }} \approx 785 \mathrm{~km} \mathrm{~s}^{-1}$, where $w$ is the fraction of the total ICM mass supplied by winds with velocities $v_{\text {wind }}$. Taking $w \approx 5$ percent (see above), then, requires that $v_{\text {wind }} \approx$ several thousand $\mathrm{km} \mathrm{s}^{-1}$, which is consistent with the superwind model $(\S \mathrm{IV} c)$.

Shapiro (1989) has recently reviewed the evidence for a general intergalactic medium (IGM) with $\Omega \approx 0.01-0.1$ and $T>$ $10^{4} \mathrm{~K}$. He has also evaluated the possible sources for heating and ionizing such an IGM, and concludes that photoionization by QSOs or young stars is inadequate by a wide margin. Here we show that superwinds could heat the IGM to $T \gg 10^{4}$ $\mathrm{K}$ and supply it with a significant amount of metals ( see also Ikeuchi and Ostriker 1986).

Using the results from $\S \mathrm{Va}$ on the global superwind injection rates of metals and kinetic energy per $L_{*}$ galaxy, we find

$$
\begin{aligned}
\xi_{\mathrm{IGM}} & \approx 0.005 \Omega_{\mathrm{IGM}}^{-1} f_{\mathrm{evol}}, \\
T_{\mathrm{IGM}} & \approx 4 \times 10^{4} \Omega_{\mathrm{IGM}}^{-1} f_{\mathrm{evol}} \mathrm{K}, \\
\Omega_{\mathrm{IGM}, \mathrm{SW}} & \approx 3 \times 10^{-4} f_{\mathrm{evol}} f_{\mathrm{entr}} .
\end{aligned}
$$

Here $\xi_{\text {IGM }}$ is the metal abundance of the IGM (in solar units) assuming that the IGM has a density $\Omega_{\mathrm{IGM}}$, that all the metal (but only a negligible amount of the mass; see eq. [18c]) in the IGM comes from superwinds, and that the cosmic evolution of 
the superwind rate increases the total injected energy and metal by a factor $f_{\text {evol }}$ relative to the no-evolution estimates in $\S \mathrm{Va}$. The effective temperature of the IGM $\left(T_{\text {IGM }}\right)$ is defined by equating the amount of kinetic energy injected by superwinds to the thermal energy content of the IGM (i.e., it ignores adiabatic and radiative cooling of the IGM). While superwinds can be important sources of metals and heat, the mass density of the IGM produced by superwinds $\left(\Omega_{\mathrm{IGM}, \mathrm{Sw}}\right)$ is small, even allowing for significant cosmic evolution and a large amount of matter entrained by the superwind from the interstellar medium or gaseous halo of the galaxy that is driving the wind (parameterized by the correction factor $f_{\text {entr }}$ ).

Finally, we consider the ultimate fate of a superwind that has blown out of its host galaxy and its expanding into the general IGM. This expansion will continue until the wind comes into pressure balance with the IGM. Following Ikeuchi and Ostriker (1986), we estimate that the IGM at $z \approx 0$ has $P / k \approx$ $10^{-4}$. Assuming adiabatic expansion of the superwinds, then, implies that the final equilibrium radius of the cavity in the IGM excavated by a superwind with a total energy of $10^{59} \mathrm{ergs}$ would be

$$
r_{\mathrm{eq}} \approx 39\left(E_{59} P_{\mathrm{IGM},-4}\right)^{1 / 3} \mathrm{Mpc}
$$

However, it will take longer than a Hubble time to reach that radius. Neglecting the expansion of the universe-for illustrative purposes only-the adiabatic Sedov solution yields the maximum radius achieved by the superwind cavity in a Hubble time:

$$
r_{\mathrm{H}} \approx 3.3 E_{59}^{1 / 5}\left(\Omega_{\mathrm{IGM}} / 0.01\right)^{-1 / 5} h^{-4 / 5} \mathrm{Mpc},
$$

where $h=H_{0} / 100$. Since $r_{\mathbf{H}}$ is comparable to typical distances between galaxies and superwinds may have produced an average of $E \approx 10^{59}$ ergs per galaxy, this suggests that the structure of the IGM may be regulated by superwinds and the cavities they create.

\section{c) Galaxy Formation and Evolution}

i) General Considerations

One of the principal motivations of studying FIRGs is the possibility of investigating "up close" the processes that may have occurred during galaxy formation. This is not quite as unrealistically romantic a notion as it might appear at first glance. An IR luminosity of $\approx 10^{12} L_{\odot}$ implies a SFR of $\approx 300$ $M_{\odot} \mathrm{yr}^{-1}$ for a Salpeter IMF. If such SFRs are sustained for galaxy free-fall times $\left(10^{8}-10^{9} \mathrm{yr}\right)$, the total mass of newly formed stars would be $\approx 10^{11} M_{\odot}$, which is similar to the mass of an $L_{*}$ galaxy in the form of stars. Thus, powerful FIRGs may indeed bear both a qualitative and quantitative resemblance to true protogalaxies.

\section{ii) "Explosions" and "Feedback" during Galaxy Formation}

It is particularly interesting to compare the superwinds we have observed to the "explosions" that Ostriker and Cowie (1981, hereafter OC) postulated to be associated with galaxy formation. These "explosions" were just very powerful superwinds: the collective effects of the multiple supernovae and stellar winds associated with the initial burst of star formation accompanying the formation of a galaxy. OC argued that as the blast waves produced by such explosions propagated out into the IGM, they could lead to the formation of galaxies and Ly $\alpha$ forest clouds as the swept-up shells of the IGM fragmented into gravitationally unstable lumps.

Our data provide general support for the OC explosion model in that our superwinds represent the efficient conversion (via star formation) of mass into kinetic energy. To estimate the amount of energy associated with an OC "explosion," we can use our observational estimates of the energy associated with the superwinds $(\S \mathrm{IV} c)$ and scale them to the level applicable during the formation of a galaxy. We found above that the mechanical energy associated with superwinds was a few percent of the bolometric luminosity of the starburst. Adopting a Salpeter IMF to relate the bolometric luminosity to the SFR, the amount of mechanical energy produced is $\approx 10^{49}$ ergs $M_{\odot}^{-1}$. Thus, the formation of a galaxy whose stellar population had a mass $\approx$ few $\times 10^{11} M_{\odot}$ would entail a few $\times 10^{60}$ ergs in mechanical energy, which is consistent with the suggestion of OC.

Dekel and Silk (1986), Silk (1987), and White (1989) have also emphasized the importance of energy input from supernovae during the process of galaxy formation. Indeed, they show that the specific binding energy of galaxies $(E / M \approx[$ few hundred $\left.\mathrm{km} \mathrm{s}^{-1}\right]^{2}$ ), and even the Faber-Jackson relation, may derive from the self-regulation of the SFR by the "feedback" provided by supernovae. Dekel and Silk (1986) further argue that only in dwarf galaxies does this self-regulation of the SFR fail, and that these galaxies, therefore, suffer catastrophic mass loss due to superwinds. Our data imply that (under the proper circumstances) galaxies that are far more massive than dwarfs can also drive large-scale outflows. Evidently, the study of the physics of local superwinds may shed some light on some of the vexing problems of galaxy formation.

\section{iii) Chemical Evolution}

We have argued that, over a Hubble time, superwinds may have propelled a mass of metals out into the IGM/ICM that is at least comparable to the mass of metals inside present-day galaxies. The escape of the metals into the IGM will occur if the wind achieves the blowout phase (MM, MMN). Alternatively, they may fall back into the extranuclear regions of the galaxy if the wind material can be confined and cooled. Norman and Ikeuchi (1989), MM, and MMN have studied qualitatively similar processes occurring in the disks of normal starforming galaxies (the "chimney," "superbubble," or "galactic fountain" models). These phenomena suggest that the chemical evolution of galaxies may be complex, with strong radial transport of metals within galaxies and possibly the wholesale export of metals out of galaxies. A consideration of some of the failings of the simple "closed box" models of the chemical evolution of galaxies (see Hunter and Gallagher 1986; Pagel and Edmunds 1981) from this perspective might be enlightening.

We also note that both the radial metallicity gradients in elliptical galaxies and the correlation between their metallicities and masses can be explained as reflecting an overall correlation between the metal abundance and the local escape veloc- 
ity (e.g., Silk 1987; Franx 1990). This result can be plausibly interpreted within the superwind context, since the degree to which a galaxy (or region within a galaxy) can retain metals ejected by evolved stars will depend in part on the local depth of the potential well (see Larson and Dinerstein 1975).

\section{iv) Merger Models for the Formation of Elliptical Galaxies}

There is a growing body of evidence suggesting that a significant fraction of bright elliptical galaxies have formed via the merger of two disk galaxies (see Toomre 1977; Schweizer 1989). The nearest 10 examples of such putative "ellipticals in the making" have been cited by Toomre (1977), and we note that eight of these meet our criteria for FIRGs: $L_{\mathrm{IR}} \geq 10^{44} \mathrm{ergs}$ $\mathrm{s}^{-1} ; L_{\mathrm{IR}} / L_{\mathrm{B}}>2 ; S_{\nu, 60 \mu \mathrm{m}}>0.5 S_{\nu, 100 \mu \mathrm{m}}$. We speculate that superwinds associated with the starbursts that are triggered during the merger help to convert the merger remnant from a gas-rich starburst galaxy into a relatively normal elliptical by sweeping out any cold interstellar clouds that remain bound to it.

We also wonder whether star formation in the shells of cold gas swept up by some superwinds might play a role in the formation of at least some of the "shells" or "ripples" found to be commonly associated with elliptical and S0 galaxies (Malin and Carter 1983; Schweizer and Seitzer 1989). Variations on this idea have been explored by Williams and Christiansen (1985) and Umemura and Ikeuchi (1987). Most of the wellstudied shells have observed properties that are more consistent with an origin as dynamically cold accreted material (e.g., Quinn 1984; Prieur 1989). However, it is intriguing that the strength of shells and related morphological peculiarities apparently correlates with the presence of a hot ("poststarburst") subcomponent in the nuclear stellar population (Carter et al. 1988; Schweizer and Seitzer 1989).

\section{v) Relationship to High-Redshift Galaxies}

We have argued above in quite general terms that FIRGs might be reasonable approximations to galaxies in formation. Since a fair number of galaxies at high redshift have now been detected, it is instructive to compare their properties to the FIRGs. The population of blue galaxies that dominate the number counts at faint magnitudes are best understood as a population of objects forming stars at a higher than average rate: they represent a population of starburst galaxies at redshifts typically ranging from several tenths to greater than 1 (e.g., Broadhurst, Ellis, and Shanks 1989; Ellis 1989; Koo 1989). These are generally strong emission-line galaxies, and as such are promising candidates in which to search for superwinds at high redshifts.

The only confirmed galaxies at the highest redshifts $(z \gg 1)$ are the radio galaxies (see McCarthy 1988; Chambers 1989). Their principal properties can be summarized as follows:

1. They are generally associated with large nebulae of ionized gas (typical size scale $30-100 \mathrm{kpc}$ ).

2. The nebulae are characterized by large internal velocities (up to $10^{3} \mathrm{~km} \mathrm{~s}^{-1}$ ).

3. The blue rest-frame colors of the galaxies imply large SFRs (about $100 M_{\odot} \mathrm{yr}^{-1}$ ).

4. The nebulae are preferentially oriented along the axis of the radio source, but the gas frequently extends well beyond the radio source.
5. The morphology of the starlight is multimodal, with the secondary "blobs" being preferentially located along the radio axis.

Properties 1-3 have clear analogs in the FIRGs, but properties 4 and 5 bear closer examination. The favored interpretation of property 4 is that the gas is photoionized by a UV continuum from the center of the galaxy that escapes anisotropically along the radio axis owing to the presence of an obscuring torus or disk whose axis of symmetry is along the radio axis. It is also possible that the nebulae are shock-heated by a bipolar superwind that is roughly coaxial with the radio jets. Such coaxiality is plausible given the observed alignment between the jet axis and the rotation axis of the large-scale $(>1 \mathrm{kpc})$ gas disks in low-redshift radio galaxies (Kotanyi and Ekers 1979; Simkin 1979; Heckman et al. 1985).

The simplest interpretation of property 5 is that the radio source has triggered the formation of an entire secondary galaxy as it expanded out through the gaseous halo of the radio galaxy. Alternatively, the star/galaxy formation may be triggered by the superwind rather than the radio source. This is in fact the central prediction of the OC "explosions" model discussed above. It would clearly be of interest to look for signs of superwind-triggered star formation in the low-redshift FIRGs we have discussed. Perhaps the supergiant $\mathrm{H}$ II regions in the nebula of NGC 6240, the $\mathrm{H}$ II regions located along the minor axes of NGC 253 and NGC 4945, and the starburst companion of NGC 3079 ( see $\S$ III) are examples of this process.

\section{vi) Protogalactic Superwinds Masquerading as High-z QSOs}

The following thought experiment suggests that some genuine protogalaxies may be hidden amoung the hundreds of high-redshift optically selected QSOs. Suppose we form the central parts of a luminous elliptical galaxy in the gravitational collapse time within this central region (i.e., convert $\approx 10^{11}$ $M_{\odot}$ into stars in $\approx 10^{8} \mathrm{yr}$ within several $\mathrm{kpc}$ of the nucleus). The bolometric luminosity of such an event would be $\approx 10^{46}$ ergs $\mathrm{s}^{-1}$, the Ly $\alpha$ luminosity (from photoionization by hot stars alone) would be $\approx 10^{45}$ ergs s$^{-1}$, and the angular size of the starburst would be $<1^{\prime \prime}$ for $z>2$. The total mechanical energy output from the supernovae and stellar winds would be $\approx 10^{60} \mathrm{ergs}$, sufficient to accelerate the entire baryonic mass of the galaxy $\left(\approx 10^{11} M_{\odot}\right)$ up to a velocity of $1000 \mathrm{~km} \mathrm{~s}^{-1}$. The shock-heated ambient gas could produce strong UV resonance lines from highly ionized species such as O VI $\lambda 1034, \mathrm{~N} v$ $\lambda 1240$, and C IV $\lambda 1549$ (Daltabuit, MacAlpine, and Cox 1978 ). The continuum from the starburst would be very blue ( $\approx$ flat in $F_{\nu}$ longward of the Lyman limit) and the UV resonance absorption lines (produced in the winds from the hot massive stars) would be broad (>1000 $\mathrm{km} \mathrm{s}^{-1}$ ) and blueshifted. Such an object might resemble those broad-absorption-line (BAL) QSOs with relatively narrow ( $<$ few thousand $\mathrm{km} \mathrm{s}^{-1}$ ) BALs (see examples in Turnshek 1988).

\section{d) The Evolution of Active Galaxies}

A number of recent papers have suggested that there may be an evolutionary relationship between starbursts and QSOs or Seyfert galaxies (Weedman 1983; Terlevich and Melnick 1985; Norman and Scoville 1988; Sanders et al. 1988; Heckman et al. 1989). In some scenarios, the starburst precedes 
(and leads directly to) the creation of the exotic object (or objects) necessary to power the QSOs or Seyfert activity. In other scenarios, the starburst and a preexisting supermassive black hole are both fueled in parallel by an external source of gas.

A common element in all these scenarios is a phase during which the dusty molecular gas that fueled the starburst is swept aside to reveal an active galactic nucleus. That is, the galaxy is predicted to evolve from a FIRG powered by a dust-shrouded starburst (and perhaps AGN) into a "classical" QSO or Seyfert galaxy. We believe that superwinds represent the physical mechanism by which the region surrounding the nucleus is swept clear of gas, and their discovery provides indirect support for these various evolutionary pictures.

We note further that a superwind precursor to the "classical" QSO or AGN phase that sweeps out the central regions of the galaxy might explain the low column densities of gas seen in front of most QSOs and luminous Seyfert 1 nuclei $(N<$ $10^{20}-10^{21} \mathrm{~cm}^{-2}$; see Reichert et al. 1985 and Canizares and White 1989).

\section{e) Superwinds and the Cosmic X-Ray Background}

As we have discussed above, superwinds should be moderately strong X-ray emitters. We will now discuss the idea ( originally suggested by Bookbinder et al. 1980) that they could make a significant contribution to the cosmic X-ray background (CXRB). This approach appears promising: Weedman (1987) has used the present-epoch far-IR luminosity function and X-ray/far-IR luminosity ratios of starburst galaxies to show that these galaxies could provide about $13 \%$ of the CXRB at $2 \mathrm{keV}$, even without any cosmological evolution. More recently, Helfand (1990) has identified a possible population of optically faint starburst galaxies with the faintest detectable X-ray sources in the Einstein deep fields. Note that X-rays from starburst galaxies could come in principle from both superwinds and X-ray binaries (see Griffiths 1989, who favors the latter).

We will estimate the maximum possible contribution of superwinds to the CXRB by positing that each gram of metals in the universe was synthesized in (and expelled from) a starburst galaxy, and each was associated with a specific amount of energy in the form of X-rays from a superwind. We will assume that this ratio of $\mathrm{X}$-ray energy to metal mass is given by the estimated/observed parameters for nearby superwind galaxies ( $\operatorname{see} \S \mathrm{IV} e$ ). The expression for the maximum possible energy density in the CXRG due to superwinds is, then,

$$
U_{x} \approx 5 \times 10^{-16}\left(1+z_{f}\right)^{-1} h^{2} \Omega_{B} \xi L_{41} \text { ergs } \mathrm{cm}^{-3}
$$

where $z_{f}$ is the mean redshift at which the metal injection occurs, $h=H_{0} / 100, \Omega_{B}$ is the fraction of the closure density in the form of baryons, $\xi$ is the metal abundance of this baryonic matter in solar units, and $L_{41}$ is the X-ray luminosity (in units of $10^{41} \mathrm{ergs} \mathrm{s}^{-1}$ ) corresponding to a metal production rate of 1 $\mathrm{M}_{\odot} \mathrm{yr}^{-1}$ in the starburst. The total energy density in the CXRB (up to energies of about $200 \mathrm{keV}$ ) is of order $10^{-16} \mathrm{ergs} \mathrm{cm}^{-3}$ (Bookbinder et al. 1980). Plugging in optimistic, but astrophysically plausible, values for the various parameters in equation
(21) (e.g., $\Omega_{B} \approx 0.1, \xi \approx 1,1+z_{f} \approx 2, L_{41} \approx 1$ ), it seems that superwinds probably cannot contribute more than about $20 \%$ to the total CXRB.

An additional problem with superwinds is that their X-ray spectrum is almost certainly too soft to match the CXRB. The latter has an effective temperature of about $4 \times 10^{8} \mathrm{~K}$ (e.g., Marshall et al. 1980), while the former are predicted (see eq. [13] above) and observed (Schaff et al. 1989) to have characteristic temperatures of order $10^{8} \mathrm{~K}$. This temperature is related to the postshock temperature attained with a shock velocity equal to the wind's terminal velocity $\left(k T=\frac{3}{16} \mu v^{2}\right.$, where $\mu$ is the mean mass per wind particle). Expressing this in convenient units gives

$$
T_{\max }=2.3 \times 10^{7} v_{3}^{2} \mu \mathrm{K},
$$

where $\mu$ is in amu and $v_{3}$ is the superwind terminal velocity in units of $1000 \mathrm{~km} \mathrm{~s}^{-1}$ (and is effectively the expansion velocity of the supernovae or stellar winds that power the superwind). Since $v_{3} \approx 2-3$ are suggested by the available data on supernovae and stellar winds, and since fully ionized gas with solar abundances has $\mu=0.6, T_{\max } \leq 2 \times 10^{8} \mathrm{~K}$. Even if the wind fluid is $50 \%$ metals by mass (EFF), then $\mu=0.9$ for the fully ionized wind fluid and $T_{\max } \leq 3 \times 10^{8} \mathrm{~K}$. Thus, superwinds could produce a hard enough X-ray spectrum only if $T=T_{\max }$ and if the CXRB is produced by low-redshift galaxies $(z \ll 1)$. The problem is more intractable if the effect of the known discrete contributors (principally QSOs) to the CXRB are subtracted: these have soft $X$-ray spectra, so the remaining CXRB is even harder than thermal emission with $T \approx 4 \times 10^{8} \mathrm{~K}$-see Giacconi and Zamorani (1987).

We conclude from the above that superwinds are not likely to account for the entire CXRB. Are they important for the $\mathrm{CXRB}$ at low energies? The energy density in the CXRB for $E \approx 1-10 \mathrm{keV}$ is a few $\times 10^{-17}$ ergs $\mathrm{cm}^{-3}$, and equation (21), then, implies that superwinds could make a significant contribution in this energy range if $z_{f}$ is not too large $\left(z_{f}<\mathrm{a}\right.$ few $)$.

\section{f) QSO Absorption-Line Systems}

The nature of the intervening clouds responsible for producing the absorption lines in the spectra of QSOs remains unclear ( see the collection of reviews in the volume compiled by Blades, Turnshek, and Norman 1988). The metal-line systems (principally the $\mathrm{Mg}$ II $\lambda 2798$ and $\mathrm{C}$ IV $\lambda 1549$ doublets) and the damped Ly $\alpha / \mathrm{H}$ r $21 \mathrm{~cm}$ systems are both believed to be associated with galaxies, with the former arising in the outer halo and the latter in the disk. In contrast, the "Ly $\alpha$ forest" lines are thought to arise in intergalactic clouds or shells. The effective cross section of absorbers per Schecter $L_{*}$ galaxy greatly exceeds the characteristic optical or $\mathrm{H}$ i dimensions of galaxies at the current epoch, with the effective diameter being 100-200 $\mathrm{kpc} \mathrm{h}^{-1}$ for the metal-line systems and $50-75 \mathrm{kpc} \mathrm{h}^{-1}$ for the damped Ly $\alpha / \mathrm{H}_{\text {I }} 21 \mathrm{~cm}$ systems. The optical nebulae associated with the most powerful FIRGs in the local universe have diameters of tens of kpc (HAM; Armus, Heckman, and Miley 1990), and are driven by starbursts with bolometric luminosities of $10^{11}-10^{12} L_{\odot}$. Could such objects and the still larger superwind nebulae that might be present around galaxies in formation produce QSO absorption lines? 
CMW have calculated the expected strength of absorption lines produced by highly ionized hot gas in the interface between the swept-up matter and the hot wind fluid in the context of bubbles blown by stellar winds. Converting their prediction for C IV into convenient units we find

$$
N_{\mathrm{C} \text { IV }} \approx 10^{13} n_{0}^{9 / 35} d E / d t_{43}^{1 / 35} t_{7}^{8 / 35} \mathrm{~cm}^{-2},
$$

where the energy flux in the superwind is given in $10^{43} \mathrm{ergs} \mathrm{s}^{-1}$, and its duration is given in units of $10^{7} \mathrm{yr}$. They give the total column density of gas in the swept-up shell as

$$
N_{\text {tot }} \approx 3 \times 10^{21} n_{0}^{4 / 5} t_{7}^{3 / 5} \mathrm{~cm}^{-2} .
$$

For an M82 class superwind these column densities only extend over scales of several $\mathrm{kpc}$. The observed $\mathrm{H}$ I column densities in the M82 nebula are of order $10^{21} \mathrm{~cm}^{-2}$ (Crutcher, Rogstad, and Chu 1978), in agreement with the above. The estimated C IV column densities implied by typical QSO absorption lines are $\leq 10^{15} \mathrm{~cm}^{-2}$, with corresponding total column densities $\leq 10^{20} \mathrm{~cm}^{-2}$. The damped Ly $\alpha / 21 \mathrm{~cm}$ systems have a low-ionization state with $N_{\mathrm{tot}} \approx N_{\mathrm{H}} \approx 10^{21} \mathrm{~cm}^{-2}$.

There are two indirect lines of evidence that suggest that superwinds might be responsible for at least some QSO metal absorption lines. First, Bergeron (1988) and Yanny, York, and Williams (1990) have discovered galaxies undergoing vigorous star formation that are apparently responsible for producing $\mathrm{Mg}$ II absorption lines in background QSO spectra. This corroborates the suggestion make by York et al. (1986). Second, the velocity structure of the metal-line systems are complex: the absorption-line profiles typically consist of several narrow subcomponents spanning a velocity range of typically several tens to several hundred $\mathrm{km} \mathrm{s}^{-1}$ (see Blades 1988). In extreme cases, velocity spreads of up to $2000 \mathrm{~km} \mathrm{~s}^{-1}$ are observed. Such substructures and velocities are a natural consequence of a line of sight that transverses a superwind.

Finally, Ostriker and Ikeuchi (1983) and Ikeuchi and Ostriker (1986) have argued that the properties of the Ly $\alpha$ forest systems are naturally understood if they are fragments of shells of intergalactic matter that has been swept up and shocked by "explosions" with energies of about $10^{61}$ ergs associated with galaxy formation (see above).

In summary, while it is by no means clear that superwinds are consistent with the myriad properties observed for the various classes of QSO absorption-line systems, we believe that superwinds are sufficiently promising to merit more detailed consideration from observers and theorists. At the very least, they may offer a novel way to transport metals out into the IGM, far from any galaxy. This transport process could perhaps alleviate some of the problems engendered by associating all the metal lines with the halos of individual galaxies.

\section{SUMMARY}

We have presented and discussed spectroscopic data on the optical emission-line gas associated with a sample of 14 far-IR galaxies (FIRGs-defined to have $L_{\mathrm{IR}}>10^{44} \mathrm{ergs} \mathrm{s}^{-1}$ and $\left.L_{\mathrm{IR}} \gg L_{\mathrm{OPT}}\right)$. These data provide solid evidence for the "superwind" concept: galaxies with very high circumnuclear SFRs have large-scale mass outflows, presumably driven by the ki- netic energy supplied by supernovae and winds from massive stars. The data support this idea in both a qualitative and quantitative fashion.

\section{a) Kinematic Evidence}

The nearest edge-on FIRGs have emission-line gas out along their minor axes whose kinematics imply outflow at velocities greater than several hundred $\mathrm{km} \mathrm{s}^{-1}$. The kinematic signature of this outflow is a kiloparsec-scale region in which the emission-line profiles are double with splittings ranging from 200 $\mathrm{km} \mathrm{s}^{-1}$ to $>1000 \mathrm{~km} \mathrm{~s}^{-1}$, suggesting an expanding shell or outflow along a conelike surface. The gas inferred to be on the side of the galaxy disk tipped slightly toward the observer is blueshifted with respect to systemic velocity. The western (brighter) " $\mathrm{H} \alpha$ bubble" in Arp 220 (HAM) is kinematically similar to the above structures, but is one order of magnitude larger. Finally, the kinematics of the spectacular emission-line nebula associated with NGC 6240 (HAM), while complex, are strongly suggestive of a bipolar outflow with the largest velocity spread (about $1000 \mathrm{~km} \mathrm{~s}^{-1}$ ) lying along the wind axis and the smallest (about $500 \mathrm{~km} \mathrm{~s}^{-1}$ ) perpendicular to it. The blueshifted emission lines (relative to systemic velocity) and the broad (about $1000 \mathrm{~km} \mathrm{~s}^{-1}$ ) blue-asymmetric wings on the emission-line profiles that characterize the central regions of FIRGs provide more general evidence for mass outflows (Armus, Heckman, and Miley 1989; Mirabel and Sanders 1988).

The size scales, velocities, and kinematic/morphological structure of the outflows all agree well with the predictions of the superwind model. The wind fluid is expected to inflate a cavity in the ISM and / or the gaseous halo of the FIRG, sweeping up ( shock heating, compressing, and accelerating) the ambient gas into a thin radiating shell. If the ambient medium is disklike, the cavity will be preferentially inflated along the minor axis, forming a bipolar nebula. The size and velocity of this expanding shell will be determined by the ambient density and the energy or momentum injection rate in the wind. The injection rates predicted by the superwind model and confirmed by our data on gas pressures (see below) and the ambient densities we derive from our data (also described below) provide excellent fits to the kinematic data.

\section{b) Evidence from Densities and Pressures}

We have been able to use the ratio of the [S II] $\lambda \lambda 6717,6731$ lines to measure electron densities $\left(n_{e}\right)$ as a function of radius for 12 of our FIRGs. The typical central densities are 500$1000 \mathrm{~cm}^{-3}$, and the densities drop smoothly with the radius, reaching $n_{e} \approx 100 \mathrm{~cm}^{-3}$ at radii of typically one to several kpc. The implied central pressures are typically few $\times 10^{-9}$ dynes $\mathrm{cm}^{-2}$, and the pressures exceed a few $\times 10^{-10}$ dynes $\mathrm{cm}^{-2}$ over regions $1-10 \mathrm{kpc}$ in diameter. The pressures we measure in FIRGs are three to four orders of magnitude higher than normal interstellar pressures and (when coupled with the modest observed X-ray luminosities of FIRGs) are inconsistent with thermal confinement by an intercloud medium that is cool enough to be gravitationally bound to the galaxy $\left(T<10^{7}\right.$ for $\left.c_{S}<v_{\text {esc }}\right)$. The isodensity/isobaric radii of the nebulae at a fiducial value $n_{e} \approx 100 \mathrm{~cm}^{-3}$ correlate well with $L_{\mathrm{IR}}^{1 / 2}$. These results are in excellent agreement with the superwind model. 
The central pressures measure the static pressure of the very hot $\left(T \approx 10^{8}\right)$, thermalized supernova/stellar wind ejecta inside the wind's sonic radius. This central pressure depends on the rates of mass and kinetic energy injection by the starburst, and on the size of the mass/energy injection region. The injection rates predicted by the superwind model yield correct central pressures for starburst radii ranging from several hundred to several thousand parsecs, in very good agreement with the actual measured sizes of the starbursts. The pressures at large radii are determined by the ram pressure of the wind and therefore measure the momentum flux injected by the starburst. They should also drop as the inverse square of the radius. The superwind model correctly predicts both the absolute value of the fiducial $n_{e} \approx 100$ radius, its dependence on $L_{\mathrm{IR}}^{1 / 2}$ (assuming that the wind momentum flux scales with the SFR, which in turn scales with $L_{\mathrm{IR}}$ ), and the shape of the radial pressure profile (shallow at small radii inside the region of momentum injection where the pressure is dominated by static thermal pressure and steepening to $r^{-2}$ at large radii where ram pressure dominates). In contrast, a wind driven by an AGN cannot match the shape of the radial pressure/density profiles because such a wind would yield an inverse square law for $P(r)$ all the way into the nucleus. The ambient (preshock) densities implied by our measured densities in the downstream $\mathrm{S}^{+}$zone are typically a few $\mathrm{cm}^{-3}$ at radii of one to several kiloparsecs.

\section{c) Evidence from Energetics and Relative Emission-Line Strengths}

The relative strengths of the emission lines in the nebulae (that is, the extranuclear gas at $r>$ few hundred pc) are similar to both the predictions made by models of shock-heated gas and to the spectra of old supernova remnants and Herbig-Haro objects (gas believed to be shock-heated). These spectra are quite different from the spectra of giant $\mathrm{H}$ II regions and from the predictions of models of gas photoionized by ordinary $\mathrm{O}$ stars. We also find that the line ratios correlate loosely with the line widths: regions with broad (narrow) lines have spectra that are most like shock-heated gas (normal giant $\mathrm{H}$ II regions). The data are therefore consistent with the superwind model, in which the kinematically disturbed gas is ambient material shock heated by the wind. Moreover, the total extinction-corrected luminosities of the extranuclear gas are energetically consistent with shock heating by the superwind. Photoionization by an AGN or any pointlike source of photons can be excluded on the basis of ionization-balance arguments. Photoionization by a spatially extended cluster of exotic "superhot" stars cannot be excluded, except probably in the NGC 3690 and Mrk 266 nebulae, where the electron temperatures in the $0^{+2}$ zone are high $(20,000-50,000 \mathrm{~K})$, favoring collisional ionization.

\section{d) Evidence from $X$-ray Nebulae in FIRGs}

The properties of the X-ray nebulae in M82, NGC 253, NGC 3628, and Arp 220 lend additional support to the superwind model. The morphologies, sizes, pressures, luminosities, masses, and temperatures agree with the model predictions in which the X-rays arise from wind fluid that has been heated to $T \approx 10^{8} \mathrm{~K}$ in an internal shock or from ambient clouds shock heated to $\approx 10^{7} \mathrm{~K}$ by the wind.

\section{e) Significance of Superwinds}

We have also discussed the possible astrophysical significance of superwinds. For a typical estimated superwind lifetime of $10^{7} \mathrm{yr}$, the total ejected mass (excluding any entrained material) is about $10^{7}-10^{9} M_{\odot}$, of which as much as $50 \%$ could be in the form of metals. The mechanical energy input to the IGM would be $10^{57}-10^{59}$ ergs per superwind. If we use the local starburst IR luminosity function and integrate over a Hubble time (with no cosmic evolution), we find that superwinds eject a gas mass of about $2 \times 10^{9} M_{\odot}$, a metal mass of $\approx 5 \times 10^{8} M_{\odot}$, and an energy of $\approx 10^{59} \mathrm{ergs}$ per $L_{*}$ galaxy. The latter two numbers are about equal to the total mass of metals in, and the binding energy of, an $L_{*}$ galaxy, respectively. If the starbursts evolve rapidly with cosmic epoch, these numbers could be one order of magnitude larger.

Superwinds may, therefore, have major ramifications for our understanding of the evolution of galaxies and the IGM. For example, they might explain the correlation between metal abundance and escape velocity in galaxies and (in particular) explain the low metal abundances and low surface brightness of dwarf galaxies. They may be the mechanism by which both the general IGM and the ICM were heated and enriched in metals, they may play a role in the origin of some QSO absorption-line systems, and they may be instrumental in the evolution of active galaxies from FIRGs to QSOs.

We have also pointed out many similarities between the properties of FIRGs and superwinds on the one hand, and both models and observations of high-redshift/young galaxies on the other. We have speculated that the properties of highredshift radio galaxies may be explained by a bipolar superwind that is roughly coaxial with the radio jet axis, and we have conjectured that some radio-quiet QSOs at high redshifts may in fact be starburst/superwinds. More generally, we have noted that building an $L_{*}$ galaxy on a galaxy dynamical time should result in an object with a luminosity similar to Arp 220 . The superwind phenomenon strongly suggests that such an event will be associated with a few $\times 10^{60}$ ergs in mechanical energy, in agreement with the "explosions" scenario of Ostriker and Cowie (1981). Such a release of energy may play a crucial role in the early evolution of the galaxy (see Silk 1987; White 1989; Mathews 1989). The superwind phenomenon should thus be included in theoretical investigations of galaxy formation.

Finally, we have used the perspective afforded by superwinds to examine the contribution that FIRGs might make to the cosmic X-ray background. While fraught with many uncertainties, our estimates indicate that FIRGs may be important contributors, particularly in the $1-10 \mathrm{keV}$ range.

We would like to thank the CTIO and KPNO mountain staff for their help in obtaining the data presented in this paper. T.M.H. and L.A. were supported in part by grants from the National Science Foundation and NASA. T.M.H. thanks Colin Norman for many useful discussions and Mike Fall for suggesting several fruitful interpretational approaches. Helpful/inspirational conversations with Jerry Ostriker, Richard Mushotzky, Paul Shapiro, Carol Lonsdale, Riccardo Giacconi, Dick McCrea, Satoru Ikeuchi, Pepi Fabbiano, Roger Chevalier, and Simon White are also acknowledged. 


\section{REFERENCES}

Antonucci, R. R. J., and Ulvestad, J. S. 1988, Ap. J. (Letters), 330, L97. Armus, L., Heckman, T. M., and Miley, G. K. 1987, Astr. J., 94, 831. 1989, Ap. J., 347, 727. 1990, Ap. J., submitted.

Axon, D. J., and Taylor, K. 1978, Nature, 274, 37

Baan, W. A., van Gorkom, J. H., Schmelz, J. T., and Mirabel, I. F. 1987, Ap. J., 313, 102.

Batchelor, R. A., Jauncey, D. L., and Whiteoak, J. B. 1982, M.N.R.A.S., 200, 19P.

Becklin, E., and Wynn-Williams, G. 1987, in Star Formation in Galaxies, ed. C. Lonsdale Persson (NASA), p. 517.

Bergeron, J. 1988, in Quasar Absorption Lines: Probing the Universe, ed. C. Blades, D. Turnshek, and C. Norman (Cambridge: Cambridge University Press), p. 127

Binette, L., Dopita, M. A., and Tuohy, I. R. 1985, Ap. J., 297, 476(BDT).

Blades, C. 1988, in Quasar Absorption Lines: Probing the Universe, ed. C. Blades, D. Turnshek, and C. Norman (Cambridge: Cambridge University Press), p. 147.

Blades, C. Turnshek, D., and Norman, C., eds. 1988, Quasar Absorption Lines: Probing the Universe (Cambridge: Cambridge University Press). Bland, J., and Tully, R. B. 1988, Nature, 334, 43.

Bookbinder, J., Cowie, L. L., Krolik, J. H., Ostriker, J. P., and Rees, M. 1980, Ap. J., 237, 647.

Bothun, G. D., Lonsdale, C. J., and Rice, W. A. 1989, preprint.

Bregman, J. 1978, Ap. J., 224, 768.

Broadhurst, T. J., Ellis, R. S., and Shanks, T. 1988, M.N.R.A.S., 235, 827

Brock, D., Joy, M., Lester, D. F., Harvey, P. M., and Ellis, H. B., Jr. 1988, Ap. J., 329, 208.

Campbell, A. 1988, Ap. J., 335, 644.

Canizares, C. R., and White, J. L. 1989, Ap. J., 339, 27.

Canto, J., Elliot, K. H., Meaburn, J., and Theokas, A. C. 1980, M.N.R.A.S. 193, 911

Canzian, B., Mundy, L., and Scoville, N. 1988, Ap. J., 333, 157

Carter, D., Prieur, J. L., Wilkinson, A., Sparks, W. B., and Malin, D. F. 1988, M.N.R.A.S., 235, 813.

Castor, J., McCray, R., and Weaver, R. 1975, Ap. J. (Letters), 200, L107(CMW)

Chambers, K. 1989, Ph.D. thesis, Johns Hopkins University.

Chevalier, R. A., and Clegg, A. W. 1985, Nature, 317, 44(CC).

Crutcher, R. M., Rogstad, D. H., and Chu, K. 1978, Ap. J., 225, 784.

Daltabuit, E., MacAlpine, G., and Cox, D. 1978, Ap. J., 219, 372.

David, L., Arnaud, K., Forman, W., and Jones, C. 1990, Ap. J., 356, 32.

Dekel, A., and Silk, J. 1986, Ap. J., 303, 39.

de Lapparent, V., Geller, M., and Huchra, J. 1988, Ap. J., 332, 44.

DePoy, D. 1987, Ph.D. thesis, University of Hawaii.

de Vaucouleurs, G., de Vaucouleurs, A., and Corwin, H. G., Jr. 1976, The

Second Reference Catalogue of Bright Galaxies (Austin: University of Texas Press).

de Vaucouleurs, G., de Vaucouleurs, A., and Pence, W. 1974, Ap. J. (Letters), 194, L119.

De Young, D. S. 1978, Ap. J., 223, 47

. 1981, Nature, 293, 43.

1986, Ap. J., 307, 62

Eales, S. A., and Arnaud, K. A. 1988, Ap. J., 324, 193.

Ellis, R. 1989, in The Edwin Hubble Centennial Symposium: The Evolution of the Universe of Galaxies, in press.

Elson, R. A. W., Fall, S. M., and Freeman, K. C. 1989, Ap. J., 336, 734(EFF)

Evans, I., and Dopita, M. A. 1985, Ap. J. Suppl., 58, 125(ED).

Fabbiano, G. 1988, $A p$. J., 330, 672.

Fabbiano, G., Heckman, T. M., and Keel, W. C. Ap. J., 355, 442.

Fabbiano, G., and Trinchieri, G. 1984, Ap. J., 286, 491.

Ferland, G. J., and Netzer, H. 1983, Ap. J., 264, 105

Ford, H. C., Dahari, O., Jacoby, G. H., Crane, P. C., and Ciardullo, R. 1986, Ap. J. (Letters), 311, L7.

Forman, W., and Jones, C. 1982, Ann. Rev. Astr. Ap., 20, 547.

Franx, M. 1990, in Proc. Heidelberg Conf. on Dynamics and Interactions of Galaxies, ed. A. Toomre and R. Wielen, in press.

Gehrz, R., Sramek, R., and Weedman, D. 1983, Ap. J., 267, 551.

Giacconi, R., and Zamorani, G. 1987, Ap. J., 313, 20.

Graham, J., Wright, G., Joseph, R., Frogel, J., Phillips, M., and Meikle, W.
1987, in Star Formation in Galaxies, ed. C. Lonsdale Persson (NASA), p. 517 .

Griffiths, R. 1989, preprint.

Gunn, J., and Gott, J. 1972, Ap. J., 176, 1.

Hacking, P., Condon, J. J., and Houck, J. R. 1987, Ap. J. (Letters), 316, L15.

Heckman, T. M., Armus, L., and Miley, G. K. 1987, A. J., 93, 276(HAM)

Heckman, T. M., Blitz, L., Wilson, A. S., Armus, L., and Miley, G. K. 1989, Ap. J., 342, 735.

Heckman, T. M., Illingworth, G. D., Miley, G. K. and van Breugel, W. 1985, Ap. J., 299, 41.

Helfand, D. 1990, private communication.

Henkel, C., Gusten, R., Downes, D., Thum, C., Wilson, T. L., and Biermann, P. 1984, Astr. Ap., 141, L1.

Ho, P., Beck, S., and Turner, J. 1990, Ap. J., 349, 57.

Hunter, D. A., and Gallagher, J. S. 1986, Pub.A.S.P., 98, 5.

Hunter, D. A., Gillet, F. C., Gallagher, J. S., Rice, W. L., and Low, F. J. 1986, Ap. J., 303, 171.

Ikeuchi, S., and Ostriker, J. P. 1986, Ap. J., 301, 522.

Irwin, J. A., Seaquist, E. R., Taylor, A. R., and Duric, N. 1987, Ap. J. (Letters), 313, L91.

Joy, M., Lester, D. F., Harvey, P. M., Telesco, C. M., Decher, R., Rickard, L. J., and Bushouse, H. 1989, Ap. J., 339, 100.

Kang, H., and Shapiro, P. R. 1989, Ap. J., submitted.

Kawara, K., Nishida, M., and Gregory, B. 1987, Ap. J. (Letters), 321, L35. Kennicutt, R. C., Jr. 1983, Ap. J., 272, 54.

-. 1988, Ap. J., 334, 144.

Kleinmann, S., Hamilton, D., Keel, W., Wynn-Williams, G., Eales, S., Becklin, E., and Kuntz, K. 1988, Ap. J., 328, 161.

Koo, D. 1989, in The Edwin Hubble Centennial Symposium: The Evolution of the Universe of Galaxies, in press.

Koski, A. T. 1978, Ap. J., 223, 56.

Kotanyi, C. G., and Ekers, R. D. 1979, Astr. Ap., 73, L1

Kraemer, S. 1986, Ph.D. thesis, University of Maryland.

Kronberg, P. P., Biermann, P., and Schwab, F. R. 1985, Ap. J., 291, 693:

Larson, R. B., and Dinerstein, H. L. 1975, Pub.A.S.P., 87, 911

Lawrence, A., Ward, M., Elvis, M., Fabbiano, G., Willner, S., Carleton, N., and Longmore, A. 1985, Ap. J., 291, 117.

Lester, D. F., Carr, J. S., Joy, M., and Gaffney, N. 1990, Ap. J., 352, 561.

Lester, D. F., Harvey, P., and Carr, J. S. 1988, Ap. J., 329, 641.

Lo, K. Y., Cheung, K., Masson, C. R., Phillips, T. G., Scott, S. L., and Woody, D. P. 1987, Ap. J., 312, 574.

Lonsdale, C. J., and Hacking, P. B. 1989, Ap. J., 339, 712.

Lonsdale, C. J., Helou, G., Good, J. C., and Rice, W. 1985, Cataloged Galaxies and Quasars Observed in the IRAS Survey (Pasadena: JPL).

Mac Low, M., and McCray, R. 1988, Ap. J., 324, 776(MM).

Mac Low, M., McCray, R., and Norman, M. 1989, Ap. J., 337, $141(\mathrm{MMN})$.

Maeder, A. 1990, in Massive Stars in Starburst Galaxies, ed. C. Leitherer, T. Heckman, C. Norman, and N. Walborn (Cambridge: Cambridge University Press), in press.

Malin, D., and Carter, D. 1983, Ap. J., 274, 534.

Marshall, F. E., Boldt, E. A., Holt, S. S., Miller, R. B., Mushotzky, R. F., Rose, L. A., Rothschild, R. E., and Serlemitsos, P. J. 1980, Ap. J., 235, 4. Mathews, W. 1989, A. J., 97, 42.

McCall, M. L., Rybski, P. M., and Shields, G. A. 1985, Ap. J. Suppl., 57, 1. McCarthy, P. J. 1988 Ph.D. thesis, University of California, Berkeley.

McCarthy, P. J., Heckman, T. M., and van Breugel, W. 1987, A. J., 93, 264(MHV).

McKee, C., and Ostriker, J. 1977, Ap. J., 218, 148.

Meurer, H.-G. R. 1989, Ph.D. thesis, Australian National University.

Mirabel, I. F., and Sanders, D. B. 1988, $A p$. J., 335, 104.

Moorwood, A. F., and Glass, I. S. 1984, Astr. Ap., 135, 281.

Morris, S., Ward, M., Whittle, M., Wilson, A. S., and Tayler, K. 1985 , M.N.R.A.S., 216, 193.

Nakagawa, T., Nagata, T., Geballe, T. R., Okuda, H., Shibai, H., and Matsuhara, H. 1989, Ap. J., 340, 729.

Nakai, N., Hayashi, M., Handa, T., Sofue, Y., Hagesawa, T., and Sasaki, M. 1987, Pub. Astr. Soc. Japan, 37, 685.

Naomasa, N. 1990, Pub. Astr. Soc. Japan, in press. 
Norman, C., and Ikeuchi, S. 1989, Ap. J., 345, 372.

Norman, C., and Scoville, N. Z. 1988, Ap. J., 332, 124.

Ostriker, J., and Cowie, L. 1981, Ap. J. (Letters), 243, L127(OC).

Ostriker, J., and Ikeuchi, S. 1983, Ap. J. (Letters), 268, L63.

Ostriker, J., and McKee, C. 1988, Rev. Mod. Phys., 60, 1.

Pagel, B. E. J., and Edmunds, M. G. 1981, Ann. Rev. Astr. Ap., 19, 77.

Pence, W. D. 1981, Ap. J., 247, 473.

Phillips, M. M., Baldwin, J. A., Atwood, B., and Carswell, R. F. 1983b, Ap. J., 274, 558.

Phillips, M. M., Turtle, A. J., Edmunds, M. G., and Pagel, B. E. J. 1983a, M.N.R.A.S., 203, 759.

Prieur, J.-L. 1989, in Proc. Heidelberg Conf. on Dynamics and Interactions of Galaxies, ed. A. Toomre and R. Wielen, in press.

Quinn, P. 1984, Ap. J., 279, 596.

Reichert, G. A., Mushotzky, R. F., Petre, R., and Holt, S. S. 1985, Ap. J., 296, 69.

Rice, W. A., Lonsdale, C. J., Soifer, B. T., Neugebauer, G., Koplan, E. L., Lloyd, L. A., de Jong, T., and Habing, H. J. 1988, Ap. J. Suppl., 68, 91.

Rieke, G. H., Cutri, R. M., Black, J. H., Kailey, W. F., McAlary, C. W., Lebofsky, M. J., and Elston, R. 1985, Ap. J., 290, 116.

Rieke, G. H., Lebofsky, M. J., Thompson, R. I., Low, F. J., and Tokunaga, A. T. 1980, Ap. J., 238, 24.

Sandage, A., and Tammann, G. 1981, The Revised Shapley-Ames Catalog of Bright Galaxies (Washington, DC: Carnegie Institution).

Sanders, D., and Mirabel, I. F. 1985, Ap. J. (Letters), 298, L31.

Sanders, D. B., Soifer, B. T., Elias, J. H., Madore, B. F., Matthews, K., Neugebauer, G., and Scoville, N. Z. 1988, Ap. J., 325, 74.

Sarazin, C. L. 1986, Rev. Mod. Phys., 58, 1.

Schaaf, R., Pietsch, P. L., Biermann, P. L., Kronberg, P. P., and Schmutzler, T. 1989, Ap. J., 336, 762.

Schiano, A. V. R. 1985, Ap. J., 299, 24.

Schweizer, F. 1989, in Proc. Heidelberg Conf. on Dynamics and Interactions of Galaxies, ed. A. Toomre and R. Wielen, in press.

Schweizer, F., and Seitzer, P. 1989, preprint.

Seaquist, E. R., Bell, M. B., and Bignell, R. C. 1985, Ap. J., 294, 546.

Shapiro, P. R. 1989, in 14th Texas Symposium on Relativistic Ap. (Ann. NY Acad. Sci.), 571, 128.

Shull, J. M., and McKee, C. F. 1979, Ap. J., 227, 122.

Silk, J. 1987, in IAU Symposium 117, Dark Matter in the Universe, ed. J.

Kormendy and G. R. Knapp (Dordrecht: Reidel), p. 335.

Simkin, S. M. 1979, Ap. J., 234, 56.
Soifer, B. T., Houck, J. R., and Neugebauer, G. 1987, Ann. Rev. Astr. Ap., 25, 1987.

Soifer, B. T., Sanders, D. B., Madore, B. F., Neugebauer, G., Danielson, G. E., Elias, J. H., Lonsdale, C. J., and Rice, W. L. 1987, Ap. J., 320, 238. Stark, A. A., and Carlson, E. R. (1984) Ap. J., 279, 122.

Taniguchi, Y., Kawara, K., Nishida, M., Tamara, S., and Nishida, M. T. 1988, Astr. J., 95, 1378.

Telesco, C. M. 1988, Ann. Rev. Astr. Ap., 26, 343.

Terlevich, R., and Melnick, J. 1985, M.N.R.A.S., 213, 831.

Thomas, P. A., Fabian, A. C., and Nulsen, P. E. J. 1987, M.N.R.A.S., 228, 973.

Tomisaka, K., and Ikeuchi, S. 1988, Ap. J., 330, 695(TI).

Toomre, A. 1977, in Evolution of Galaxies and Stellar Populations, ed. B. M. Tinsley and R. B. Larson (New Haven: Yale University Observatory), p. 401.

Turner, B. E. 1985, Ap. J., 299, 312.

Turner, J., Martin, R., and Ho, P. 1990, Ap. J., 351, 418.

Turnshek, D. 1988, in Quasar Absorption Lines: Probing the Universe, ed. C. Blades, D. Turnshek, and C. Norman (Cambridge: Cambridge University Press), p. 17

Ulrich, M.-H. 1978, Ap. J., 219, 424

Umemura, M., and Ikeuchi, S. 1987, Ap. J., 319, 601.

van Gorkum, J., and Kotanyi, C. G. 1985, in ESO Workshop on the Virgo Cluster, ed. O.-G. Richter and B. Binggeli (Munchen: ESO), p. 61.

Ward, M. 1988, M.N.R.A.S., 231, 1P.

Watson, M. G., Stanger, V., and Griffiths, R. E. 1984, Ap. J., 286, 144.

Weaver, R., McCray, R., Castor, J., Shapiro, P., and Moore, R. 1977, Ap. J., 218, 377.

Weedman, D. W. 1983, Ap. J., 266, 479.

Weedman, D. W. 1987, in Star Formation in Galaxies, ed. C. J. Lonsdale Persson (NASA), p. 351.

White III, R. E. 1990, preprint.

White, S. 1989, in The Epoch of Galaxy Formation, ed. C. Frenk, R. Ellis, T. Shanks, A. Heavens, and J. Peacock (Dordrecht: Kluwer), p. 15.

Whiteoak, J. B., and Gardner, F. F. 1979, Proc. Astr. Soc. Australia, 3, 319. Williams, R. E., and Christiansen, W. A. 1985, Ap. J., 291, 80.

Wilson, A. S., Baldwin, J., and Ulvestad, J. 1985, Ap. J., $291,627$.

Yanny, B., York, D., and Williams, T. 1990, Ap. J., 351, 377.

York, D. G., Dopita, M., Green, R., and Bechtold, J. 1986, Ap. J., 311, 610.

Young, J. S., Claussen, M. J., and Scoville, N. Z. 1987, Ap. J., 324, 115.

Lee Armus and Timothy M. Heckman: Department of Physics and Astronomy, Johns Hopkins University, Homewood Campus, Baltimore, MD 21218

GeORGe K. Miley: Sterrewacht Leiden, Postbus 9513, 2300RA, Leiden, The Netherlands 